\title{
Design and Testing of an Acoustic Ultra-short Baseline Navigation System
}

by

\author{
Brian Hearne Tracey \\ B.A., Physics \\ Kalamazoo College (1988)
}

Submitted in partial fulfillment of the requirements for the degree of MASTER OF SCIENCE IN OCEANOGRAPHIC ENGINEERING at the

MASSACHUSETTS INSTITUTE OF TECHNOLOGY and the

WOODS HOLE OCEANOGRAPHIC INSTITUTION

September 1992

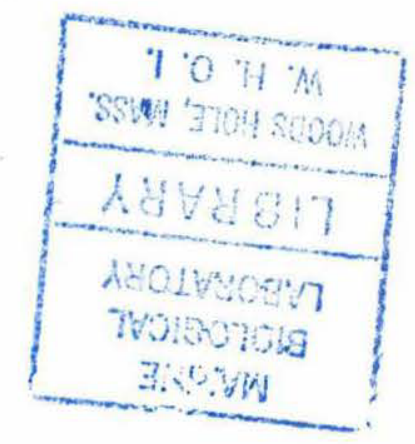

(c) Brian H. Tracey, 1992. All rights reserved.

The author hereby grants to M.I.T. and W.H.O.I. permission to reproduce and to distribute copies of this thesis document in whole or in part.

Signature of Author

$$
\text { Joint Pfggram in Oceanographic Engineering }
$$
July 30,1992

Massachusetts Instifute of Tęhnology/Woods Hele Oceanographic Institution

Certified by

$\widehat{\text { Dr. James G. Bellingham }}$

Technical Supervisor

Certified by

Prof. Henrik Schmidt

Thesis Supervisor, Massachusetts Institute of Technology

Certified by

Dr. Subramaniam Rajan

Woods Hole Oceanographic Institution

Accepted by

Prof. Arthur Baggeroer

Chairman, Joints Gramittee for Oceanographic Engineering Massachusetts Institute of Techsology Wônds Hole Oceanographic Institution

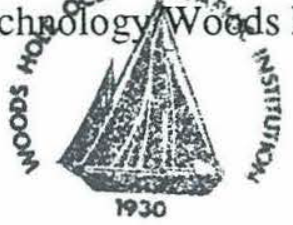




\title{
Design and Testing of an Acoustic Ultra-short Baseline Navigation System
}

\author{
by \\ Brian Hearne Tracey
}

\author{
Submitted to the Department of Ocean Engineering \\ on 30 July 1992 in partial fulfillment of the requirements for the \\ Degree of Master of Science in Ocean Engineering
}

\begin{abstract}
An ultra-short baseline acoustic navigation system has been developed which is capable of determining bearing to a sound source with an error of less than $1^{\mathrm{O}}$ in typical operational conditions. The system has a demonstrated ability to operate in an environment in which multipath interference is significant. A DSP microprocessor is used to process signals received by two hydrophones from a $26 \mathrm{kHz}$ toneburst sound source. This processing power is used to implement features not commonly available with commercial systems. The system has the capability to make an on-line measurement of the signal-tonoise ratio, which can be used to estimate the confidence which should be placed in the data. Estimates of phase difference and signal power are generated many times within each received pulse, so the effects of multipath interference throughout the pulse can be observed. Results of tests at several ranges are presented, and compared to performance models developed in the thesis. System performance is quantified, and an effort is made to understand the effects of multipath arrivals.
\end{abstract}

Technical Supervisor: James G. Bellingham

Title: Research Engineer, MIT Sea Grant Underwater Vehicles Lab

Thesis Supervisor: Henrik Schmidt

Title: Professor of Ocean Engineering 


\section{Acknowledgments}

I would never have been able to complete this thesis without the help of many people, who I would like to thank again here. First I would like to thank my two advisors,

Jim Bellingham and Henrik Schmidt, for their many insights and encouraging words along the way. I am very grateful to MIT Sea Grant for supporting this work. Bill Foley at the Charlestown Navy Yard deserves many thanks for facilitating our use of the test site there, as does Dr. Rajan at WHOI for reading my thesis on such short notice. I owe a large debt to the staff at Sea Grant, especially Tom Consi, without whom the electronics would never have been built. Jim Bales, Cliff Goudey, and John Leonard helped by letting me bounce ideas off them, and Michael Perrier amazed me by eating giant quantities of Cheese Whiz. Peter Khoury's energy and debugging abilities really helped to bring the in-water testing together - I'll pay you back this fall. I am also grateful to the staff of the Draper Labs sonar group, especially John Irza, John Halsema, and Ken Rolt. Ken's knowledge of practical sonar systems was invaluable on many occasions, and John Halsema would probably notice a definite similarity between my test rig and his. Perhaps more important than the technical assistance given to me by the people above is the fact that I consider many of them to be my friends. Engineering seems to have as much to do with working with people as with technology, and I've been fortunate to work with people who I like and respect. That goes also for the grad and undergrad students in the lab - Diane, Drew, Max, Cedric, Dave, Omar, and just about everybody else (except for Elias, of course).

I've also had a life outside the lab, and I'd like to thank a lot of people for helping me keep everything in perspective. Ram and the Boneheads at 41 Clarendon have been great to live with, so even though I won't miss the kitchen floor I'll miss the company. Amy, Steve, Jen, Bruce, and Susan all deserve special thanks. Last, but definitely not least, Laura did an amazing job of putting up with me while I went through quals, tried to decide what to do with my life, and wrote this thesis, so thank you. 
Dedicated to my parents, Don and Alice, whose love, support, and willingness to pay long distance phone bills have kept me going for many years. 


\section{Table of Contents}

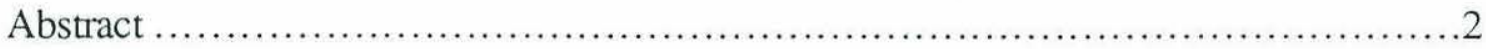

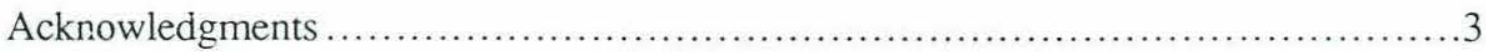

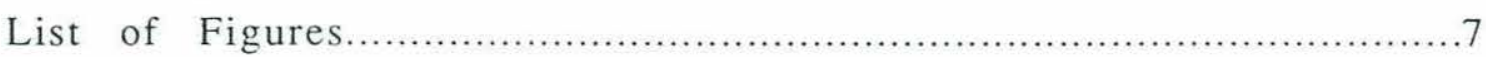

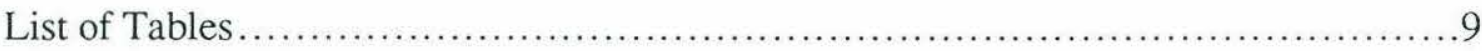

I. Introduction to USBL Applications and Techniques............................. 10

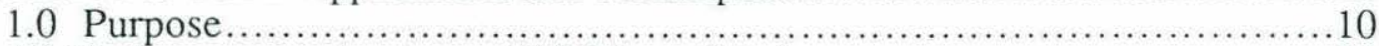

1.1 Underwater navigation and USBL applications .......................... 10

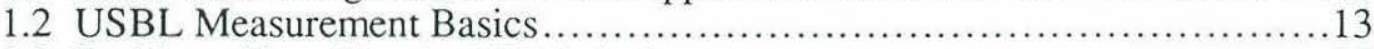

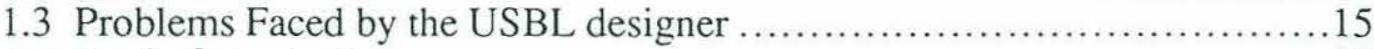

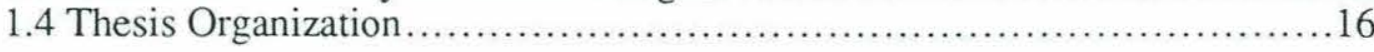

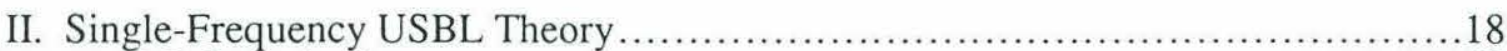

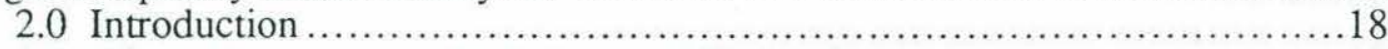

2.1 USBL Measurement Technique and Design Issues ......................18

2.1.1 Generalized USBL Measurement ............................. 18

2.1 .2 Hydrophone Array Geometry .................................23

2.1.3 Relationship of USBL Measurement to Conventional

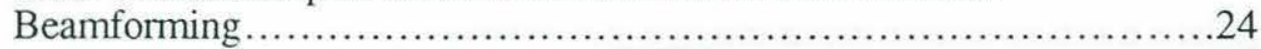

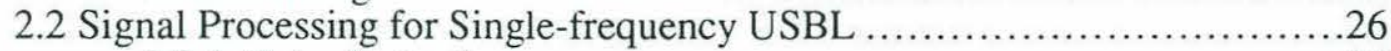

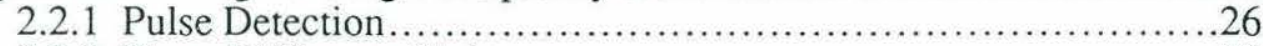

2.2.2 Phase Difference Estimate ...................................28

2.2.2.1 Maximum Likelihood Estimation of Phase

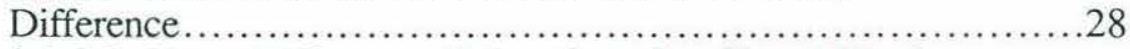

2.2.2.2 Phase Difference Estimation after Signal Hard-

limiting .................................................... 31

2.2.3 Calculation of Power and On-line Signal-to-Noise Estimate ........33

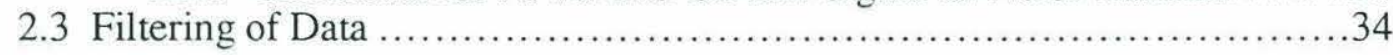

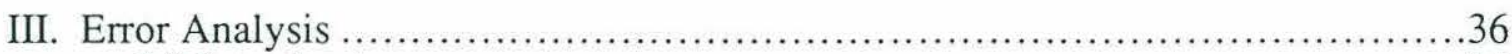

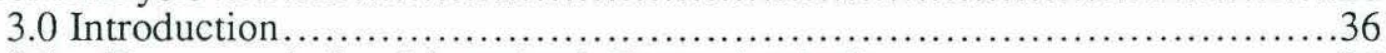

3.1 Framework for Linearized Error Analysis......................................36

3.1.1 Bearing Errors caused by Uncertainty in Time/Phase

Difference Estimate ............................................... 37

3.1.2 Bearing Errors caused by Errors in Assumed Sound Speed.........37

3.1.3 Bearing Errors caused by Uncertainty in Hydrophone

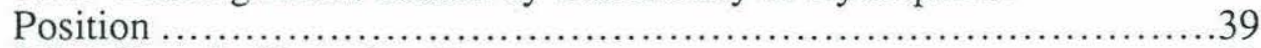

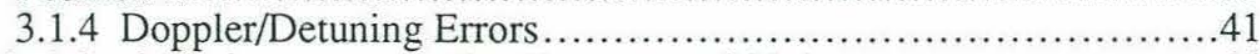

3.2 Model of Performance in the Presence of Noise...................................44

3.3 Comparison with Hard-limited USBL Systems .........................47

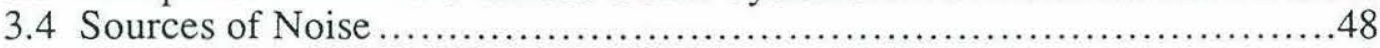

3.4.1 Analog Circuitry Noise................................................4

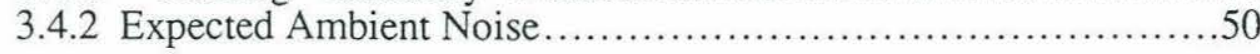


3.4.3 A/D Quantization Noise ....................................51

3.5 Predictions of Bearing Estimation Performance vs. Range .................52

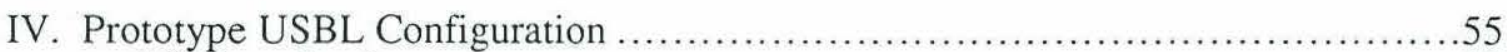

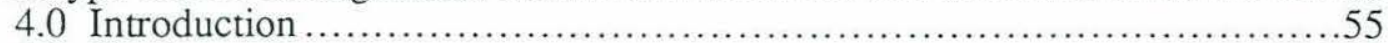

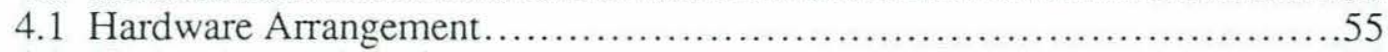

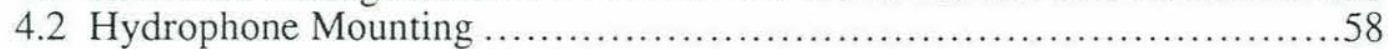

4.3 Description of Acoustic Environment ...................................60 60

4.4 Sound Speed Measurement and Ray Trace Results .......................63

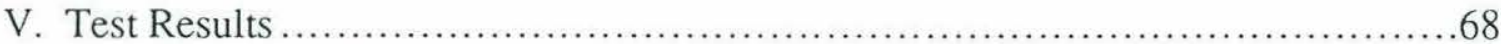

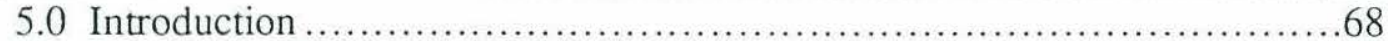

5.1 Overview of Experimental Program ....................................68

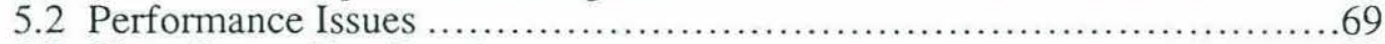

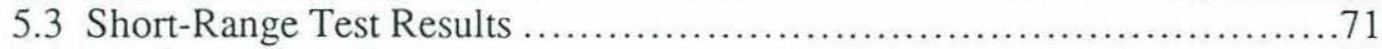

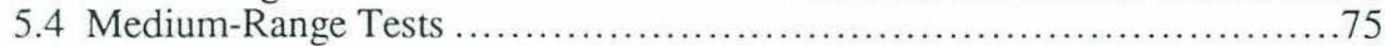

5.5 Long-Range Tests............................................... 79

5.6 Correlation between Fluctuations in Signal Power and Bearing.............83

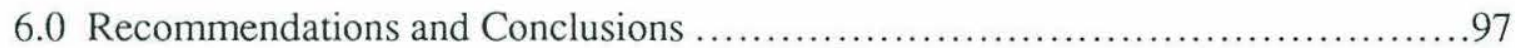

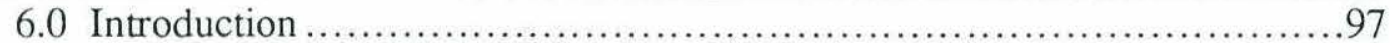

6.1 Prototype System Performance .........................................97

6.2 Recommendation for Hardware Improvements ..........................98

6.3 Recommendations for Algorithm Improvements ........................99

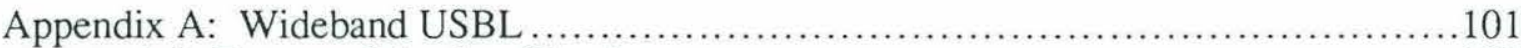

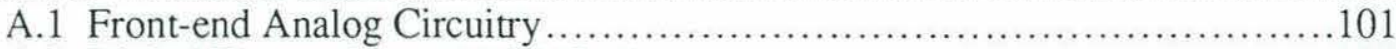

A.2 Digital Electronics and DSP Coding .................................. 111

A.3 In-Lab Noise Performance Measurements ............................ 116

Appendix B: All-Band Vs. In-Band Noise..................................... 125

Appendix C: Phase Interference of Two Superposed Multipath Arrivals ...............129

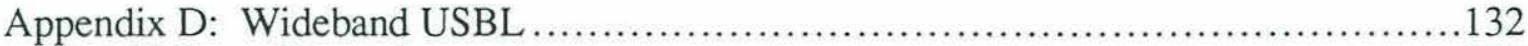

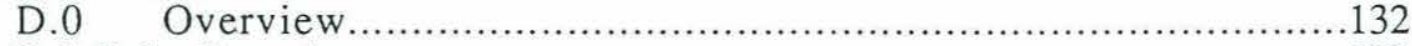

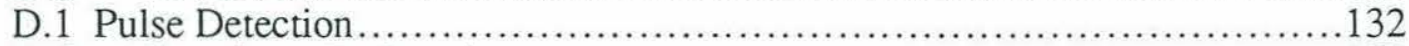

D.2 Bearing Estimation...................................................

D.2.1 Signal Processing ........................................ 133

D.2.2 Bearing Error Predictions ..................................... 135

D.3 Performance Improvements from Sensor Spacing ...........................137

D.4 Possible Development for USBL system .............................. 138 


\section{List of Figures}

Figure 1.1: Proposed Arctic Navigation Scheme...........................................12

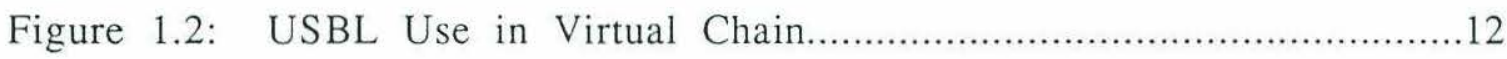

Figure 1.3: USBL Measurement for Single Baseline .............................. 14

Figure 2.4: Coordinate System for Three-Dimensional USBL Array ...................19

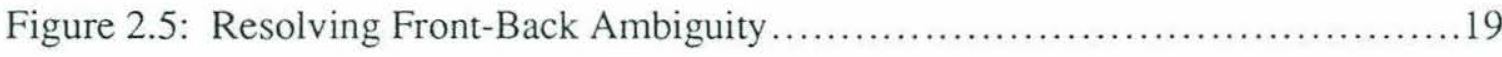

Figure 2.6: Cone of Constant Phase Difference ....................................22

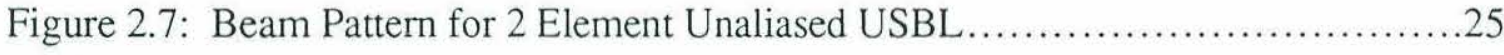

Figure 2.8: Delay-and-Sum Direction Finding for a 2 Element Array ...................26

Figure 2.9: Phase Difference Estimation Using Exclusive-OR ......................... 32

Figure 3.10: Bearing Error Resulting from Error in Phase Difference .................38

Figure 3.11: Bearing Error Resulting from Error in Sound Speed Estimate..............38

Figure 3.12: Bearing Error Resulting from Errors in Baseline Length (X-direction).....40

Figure 3.13: Bearing Error Resulting from Hydrophone Offset in Y......................40

Figure 3.14: Hydrophone Position Coordinate System ...............................41

Figure 3.15: Measured Vs Calculated Doppler Errors...............................43

Figure 3.16: Measured Vs Calculated Offsets due to Doppler.........................43

Figure 3.17: Geometric Model for Effects of Noise on One Channel ..................45

Figure 3.18: Measured and Calculated Phase Difference Errors Vs SNR..................47

Figure 3.19: Ambient Noise Power Spectra for Shallow Water .......................51

Figure 3.20: Predicted Signal-to-Noise Ratio Vs Range ..............................53

Figure 3.21: Predicted Bearing Error Vs Range for Small Bearing Angles..............54

Figure 4.22: Pinger and Hydrophone Deployment .................................56

Figure 4.23: Front-End Electronics Configuration........................................57

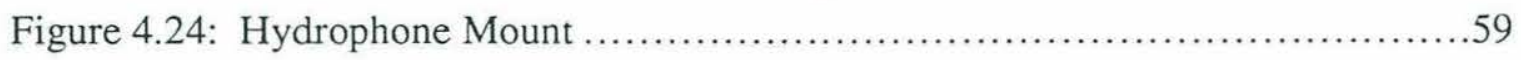

Figure 4.25: Experimental Setup at Charlestown Navy Yard...............................61

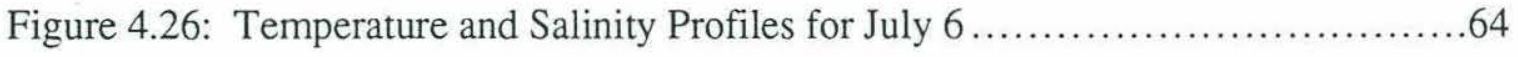

Figure 4.27: Typical Sound Speed Profiles..............................................65

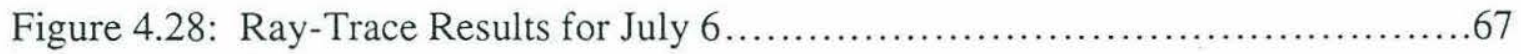

Figure 5.29: Bearing Measurement Results for May 21 Short-Range Tests.............72

Figure 5.30: Effects of Out-of-Plane Corrections on May 15 Results..................73 
Figure 5.31: Bearing Measurement Results for Medium-Range Tests ...............75

Figure 5.32: Results for Bottom-Facing Pinger Configuration...........................77

Figure 5.33: Results for Surface-Facing Pinger Configuration ...................78

Figure 5.34: Bearing Measurement Results for Long-Range Tests..................79

Figure 5.35: Results for 125 Meter Range at $45^{\circ}$ Bearing ........................ 82

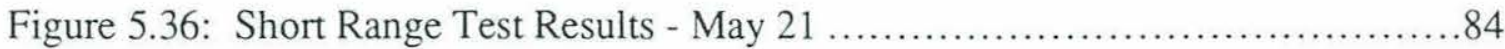

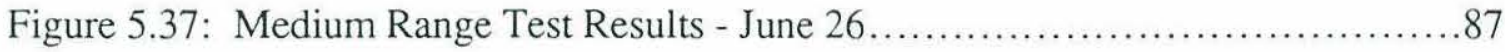

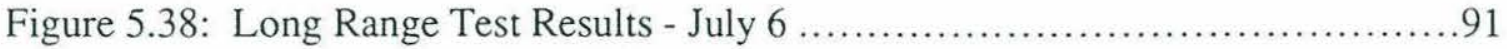

Figure A.1: Front-End Electronics Configuration.................................... 102

Figure A.2: Filter Module Specifications......................................... 105

Figure A.3: Butterworth Filter Characteristics...................................106

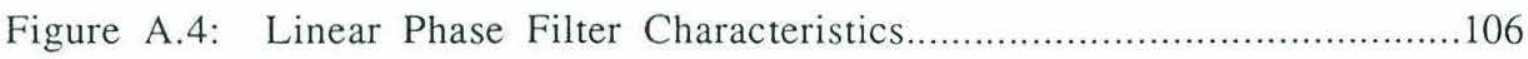

Figure A.5: Programmable Filter Characteristics....................................107

Figure A.6: Grounding and Shielding for Front-end Electronics $\ldots \ldots \ldots \ldots \ldots \ldots \ldots \ldots 110$

Figure A.7: Diagram for Data Acquisition Circuit..........................................113

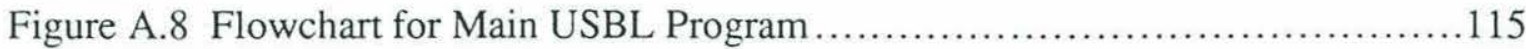

Figure A.9 Signal-to-Noise Test Input Generator......................................117

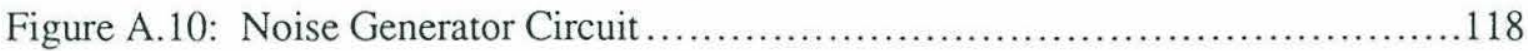

Figure D.1: Time Delay Estimation Using Spectral Estimate ........................134

Figure D.2: Predicted Bearing Error Vs Range for Different Signal Bandwidths........136

Figure D.3: Effect of Spatial Aliasing on Wideband Phase Difference .................137

Figure D.4: Bearing Error Vs Range for 15-25 kHz Signal, Different Sensor

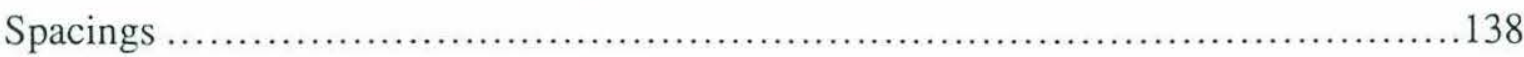




\section{List of Tables}

Table 3.1: Noise Levels for Analog Front-End Circuitry ..........................49

Table 4.1: Expected Ray Paths for Test Geometries.............................66

Table 5.1: Measured and Predicted Estimation Errors ..............................70

Table 5.2: Calculated Correlations Between Power and Bearing Fluctuations in 5

Pulses .................................................................. 83

Table A.1: Datasonics ALP-363 Pinger Specifications.............................. 101

Table A.2: Benthos AQ-4 Hydrophone Specifications ........................... 103

Table A.3: Ithaco 144N Preamplifier Specifications ............................. 103

Table B.1 Measured Signal Powers and Ratios ................................. 128 


\section{Introduction to Ultra-short Baseline Applications and Techniques}

\subsection{Purpose}

The performance and flexibility of Ultra-Short Baseline (USBL) acoustic navigation systems can be enhanced through the use of powerful digital signal processing microprocessors, coupled with an understanding of the acoustic environment. This thesis describes the design of a prototype USBL system. In-water test results are discussed, and methods for using the system's processing power to improve system performance are explored.

\subsection{Underwater navigation and USBL applications}

Reliable underwater navigation is currently one of the largest stumbling-blocks for the development of autonomous underwater vehicles (AUV's). The problems of working in the underwater environment and the size and power constraints forced on the designer by the vehicle mean that many conventional navigation systems have serious disadvantages. Radio-based navigation systems fail as radio waves cannot penetrate more than a few meters into salt water. Dead reckoning systems can be used, but generally have no way to correct for drift caused by the presence of currents. Inertial navigation systems which are affordable for scientific missions typically have drift rates on the order of $0.5-10 \mathrm{ft} / \mathrm{sec}$ [Huiyong, 1984] and are therefore inappropriate, although new developments in ring-laser gyros seem promising. Vehicle-mounted active Doppler sonars can be useful for nearbottom missions, but their size and power requirements can pose problems for small untethered vehicles. Navigation systems such as geophysical map matching may be important in the future, but geophysical features are not mapped in many parts of the ocean.. Given the available choices, it can be seen that acoustic navigation is an important tool for underwater navigation [Schmidt, Bellingham, and Consi, 1990]. Although there 
are many problems inherent in designing a reliable, accurate acoustic navigation system, it is possible to build small, low power systems which can provide adequate coverage for a survey area.

MIT Sea Grant is developing versions of two of the most commonly used types of acoustic navigation, long baseline (LBL) and USBL. Long baseline navigation systems consist of three or more acoustic beacons which are deployed to form a transponder net in the survey area. A vehicle operating inside the transponder net receives pulses from each beacon on a single hydrophone. After time synchronization problems are accounted for, the vehicle can use the times of arrival of the pulses to determine its range from each beacon, and thus locate itself inside the net [Bellingham, 1991]. While LBL is a very useful system, the process of deploying and surveying the transponder net can be timeconsuming. USBL addresses this drawback by turning the problem on its head; the system now consists of a single beacon in the water, and multiple hydrophones on the vehicle. By comparing the signals received on the different hydrophones the system measures the bearing of the pinger relative to the hydrophone array. If clocks on the beacon and vehicle can be accurately synchronized, the time of arrival of the pulse can be used to obtain range information as well. However, even without clock synchronization the USBL system can be quite useful, for example for homing in on a beacon.

The use of the USBL system can be more clearly illustrated by considering the proposed navigation system for the Arctic ice mapping mission. A series of acoustic experiments to study ice mechanics are planned for summer 1994. These experiments will focus on the events surrounding the formation of ice features and the collision of ice plates. Researchers, along with the AUV Sea Scout, will be based at a main camp. When ice activity is detected, researchers will fly to the active site to deploy acoustic sensors and an LBL array. The AUV will be launched from the base camp at about the same time. The Sea Scout will first navigate from the base camp to the active site, map the underside of the ice at the active area using sector scan and sidescan sonar, then return to 

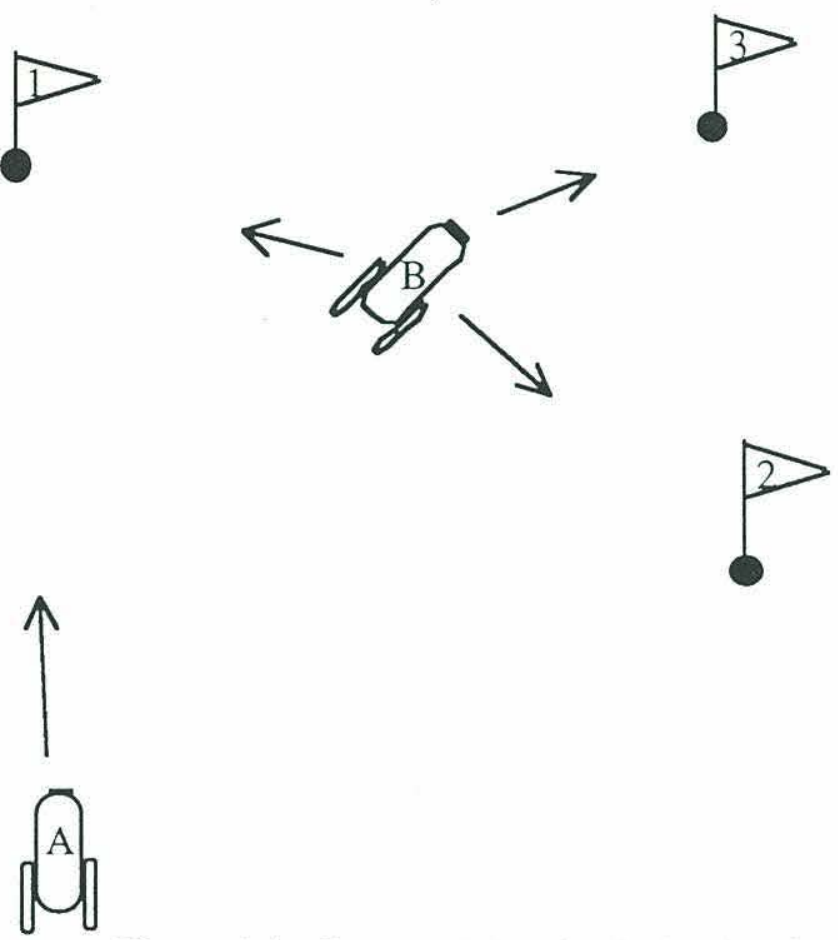

Figure 1.1: Proposed Arctic Navigation Scheme

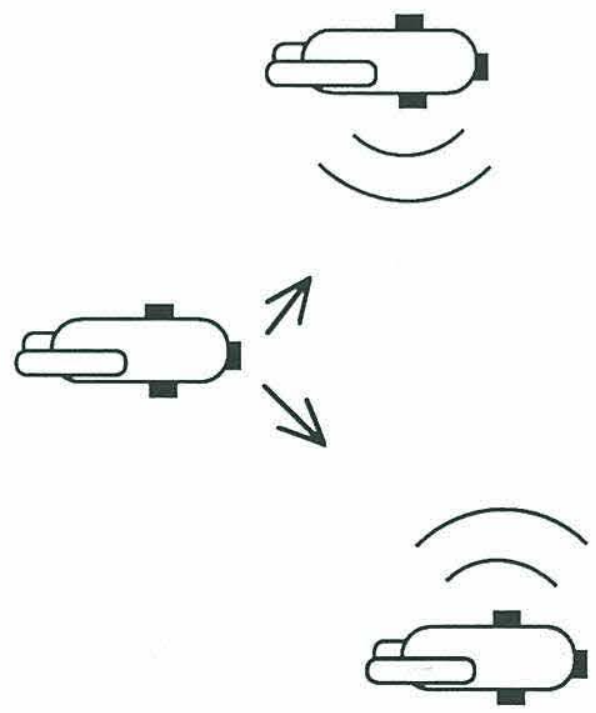

Figure 1.2: USBL use in Virtual Chain 
the base camp. Since all operations will be carried out under ice, reliability is very important. The solution which has been proposed is shown below in Figure 1.1. After leaving the base camp the vehicle will use USBL to home in on one of the beacons deployed near the active site (vehicle A below). This eliminates the need to deploy an LBL array along the route, resulting in faster deployment time. During the survey the LBL system will be used. At the same time the USBL system can provide estimates of the vehicle's bearing to each of the LBL beacons, giving an additional position estimate (vehicle B). Perhaps more importantly, the bearing measurement can be used to determine the vehicle's heading. This is especially important since magnetic compasses are not reliable near the Earth's magnetic pole, since the lines of magnetic flux have only a small horizontal component. After the survey the vehicle uses USBL to home in on a beacon located at the base camp [Schmidt and Bellingham, 1991].

Another interesting application for a USBL system is the "virtual chain" concept for control of multiple AUV's [Triantafyllou and Streitlien, 1991]. In the virtual chain, a large number of AUV's are stacked on top of one another to provide sampling through the water column. Each AUV acts as a link in a chain, attempting to remain directly in between the vehicles above and below it. The motion of the AUV line can be described as the motion of a chain, and the vehicle at the top or bottom can control the chain by acting as a forcing function. The shape of the chain will also give information about current profiles. If each vehicle is equipped with a pinger of a different frequency, a USBL system can be used to locate the AUV's above and below a particular vehicle. This is seen in Figure 1.2.

\subsection{USBL Measurement Basics}

A good physical understanding of how the USBL measurement is made can be arrived at by considering a single hydrophone pair oriented along the y axis, which receives an incident wave in the $\mathrm{x}-\mathrm{y}$ plane. If the baseline length $\mathrm{D}$ and the local sound speed $\mathrm{c}$ are 
known, and the difference between the time of arrival at the different hydrophones $\delta t$ is measured, the bearing $\theta$ is found using simple trigonometry:

$$
\theta=\operatorname{Arcsin}\left(\frac{\mathrm{c} \delta \mathrm{t}}{\mathrm{D}}\right)
$$

In the case of wideband USBL, which will be discussed in Appendix C, the time difference $\delta t$ is measured using spectral estimation techniques. For single-frequency USBL, however, a simpler expression can be derived by noting that a time delay between two sinewaves of the same frequency can also be described as a phase shift between the signals. Mathematically,

$$
\frac{\delta \mathrm{t}}{\mathrm{T}}=\frac{\Delta \phi}{2 \pi}
$$

where $T$ is the period of the sinewave and $\Delta \phi$ is the phase difference. The expression for $\theta$ above can be rewritten:

$$
\theta=\operatorname{Arcsin}\left(\frac{\Delta \phi \lambda}{2 \pi \mathrm{D}}\right)
$$

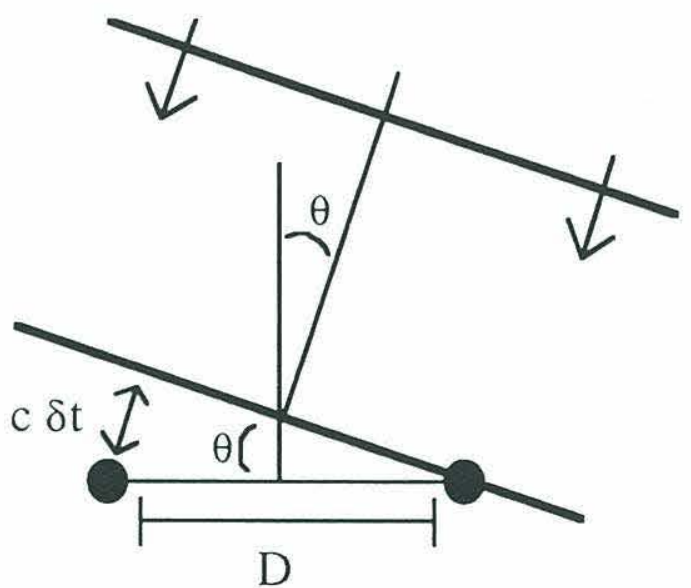

Figure 1.3: USBL Measurement for Single Baseline

In Chapter 2 the full USBL measurement technique will be explained. Typically, useful USBL systems will have at least two baselines, which can be formed using three hydrophones. Although this extension is important, all the important elements of the bearing estimation problem are encountered with a single-baseline system. This thesis 
therefore concentrates on solving the single-baseline bearing estimation problem, and suggests how additional sensors might be added to improve performance.

\subsection{Problems Faced by the USBL designer}

There are many engineering challenges which must be overcome before an accurate and reliable USBL system can be created. Some of these problems, for example accurate hydrophone mounting and reduction of electronic circuit noise, can be overcome through careful design and construction. The most interesting and challenging problems, however, require a good understanding of the acoustic environment in which the system is to operate. This understanding will affect the signal processing implementation chosen.

Any sonar system will have its performance degraded by the presence of ambient noise. Ambient noise in the ocean arises from a number of sources, including wind-water interactions, shipping noise, and biological noise. Expected background noise levels will be calculated in Chapter 3, and the effects of noise on system performance will be discussed. It will be shown there that for the prototype system, the electronics self-noise is stronger that ambient noise. Thus ambient noise has no significant effect on performance for the current implementation.

The most difficult problem with working in the ocean environment results from the multiple paths that may exist between the acoustic beacon and the vehicle. Multipaths in shallow water generally consist of a direct path arrival followed by surface- and bottomreflected arrivals. At longer ranges and in deeper water the effects of refraction become more important, and the first arrival may no longer be along the direct path. In deep water the multiple paths may all be refracted paths. Work is being done at MIT Sea Grant to model the ocean environment and extract information from multipath effects [Deffenbaugh, 1992]. This work will presumably be incorporated into the USBL system. For the purposes of this thesis, however, the assumption is made that the range is small enough that the direct path will be the first arrival. 
Multipath presents much more of a problem to the USBL designer when two or more paths are present which arrive almost simultaneously, overlapping each other for part of their duration. During the overlapped time the two arrivals will interfere with each other, destroying the phase information which is needed for the USBL measurement. It is then necessary to make some judgment as to which, if any, part of the pulse contains uncontaminated signal which can be used. One of the attractive features of the singlefrequency system built for this thesis is that it provides the possibility of making this judgment. Many commercial systems, in contrast, make assumptions about when multipath will be present and use only data from a preset section of the pulse. This approach will result in accepting bad data in some circumstances, and rejecting good data in others..

Refraction of sound can also lead to the formation of shadow zones which will not be insonified by the beacon. The only solutions to this problem are to deploy the beacon at a depth where no shadow zones will develop for the area of operation, or to incorporate position estimates from dead reckoning or some other navigation sensor in a tracking system so the vehicle can operate without USBL position inputs for some time.

\subsection{Thesis Organization}

This chapter has presented many of the issues involved in designing a USBL system. In Chapter 2 the USBL measurement is discussed in more detail, and the effects of hydrophone geometry are considered. The signal processing implementation for the prototype USBL is explained, and is compared with the more conventional hard-limiting approach. Chapter 3 presents an analysis of sources of error in the system. A model for system performance in the presence of noise is presented. Chapter 4 describes the experimental set-up used for in-water tests, and examines problems posed by the acoustic environment. Experimental results obtained from in-water tests are presented and discussed in Chapter 5. Technical data on the electronics used for implementation is 
contained in the appendices. In Appendix D, the possibility of using wideband pulses for a USBL system is explored. 


\section{Single-Frequency USBL Theory}

\subsection{Introduction}

In this chapter the USBL measurement technique is described in more detail. The brief introduction given in Chapter 1 is extended and the cases of several baselines and alternative hydrophone positionings are examined. The signal processing implementation developed in this thesis is presented, with special attention paid to pulse detection, bearing estimation, on-line signal-to-noise ratio (SNR) estimation, and data filtering. This processing implementation is contrasted with approaches commonly taken in commercial USBL systems.

\subsection{USBL Measurement Technique and Design Issues}

As noted in Chapter 1, the USBL technique provides a method for measuring the angle at which an incident sound wave strikes an array of receivers. The following discussion should make clear how the measurement is done, and introduce some of the issues which apply for any implementation of a USBL system. Some issues, such as system performance in the presence of noise and multiple arrivals, depend more on the type of algorithm used and will be discussed later where appropriate. The effects of hydrophone geometry will be discussed.

\subsubsection{Generalized USBL Measurement}

The most general USBL setup is seen below in Figure 2.4. The receiving system consists of three pairs of hydrophones. The hydrophones in each pair are separated by a distance $\mathrm{D}$, which is typically less than a wavelength. If the sound source is far away from the hydrophones, it is safe to ignore curvature in the wavefronts and assume plane 


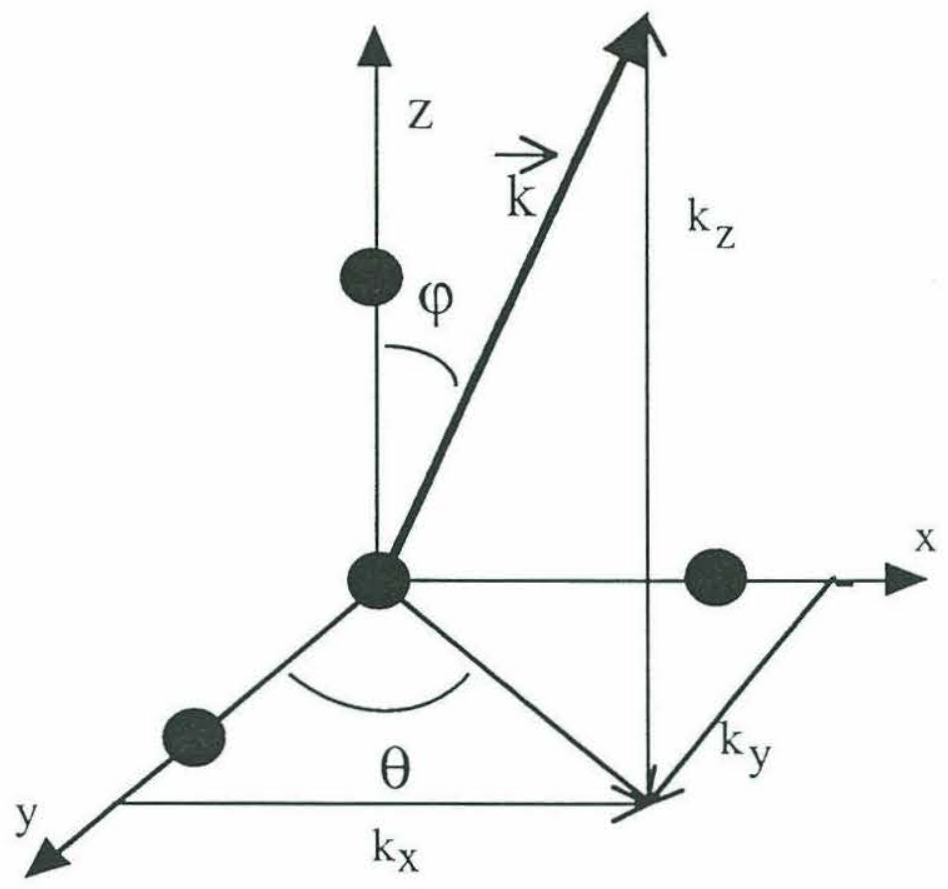

Figure 2.4: Coordinate System for Three-Dimensional USBL Array

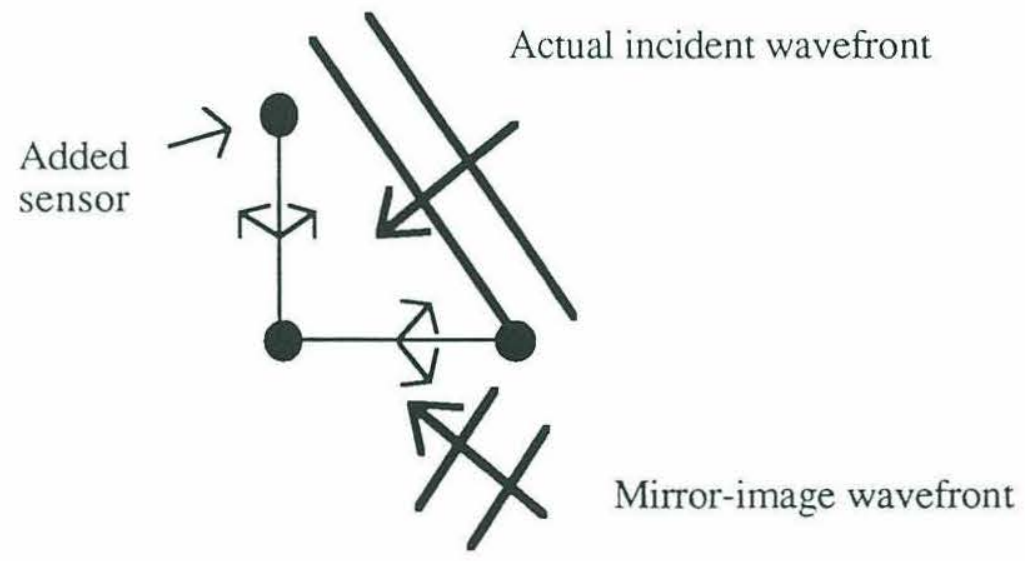

Figure 2.5: Resolving Front-Back Ambiguity 
waves are incident on the array. The propagation of plane sound waves is described by the equation

$$
\mathrm{p}(\overrightarrow{\mathrm{r}}, \mathrm{t})=\mathrm{p}_{0} \sin (\overrightarrow{\mathrm{k}} \cdot \overrightarrow{\mathrm{r}}+\omega \mathrm{t})
$$

where the direction of propagation is described by the wavenumber $\overrightarrow{\mathrm{k}}$. The sound source can transmit a single tone, described by $\mathrm{Eq}(1.1)$, or a wideband pulse containing many sinusoidal components in some frequency band (see Appendix C). In any case, the wavenumber can be decomposed into $\mathrm{x}, \mathrm{y}$, and $\mathrm{z}$ coordinates as seen in Fig. 2.4. Specifically,

$$
|\vec{k}|=\frac{\omega}{c}=\sqrt{k_{x}^{2}+k_{y}^{2}+k_{\frac{z}{2}}^{2}}
$$

where [Hildebrand,p.312]

$$
\begin{gathered}
\mathrm{k}_{\mathrm{x}}=\overrightarrow{\mathrm{k}} \sin \varphi \cos \theta \\
\mathrm{k}_{\mathrm{y}}=\overrightarrow{\mathrm{k}} \sin \varphi \sin \theta \\
\mathrm{k}_{\mathrm{z}}=\overrightarrow{\mathrm{k}} \cos \varphi
\end{gathered}
$$

When the plane wave strikes the hydrophone array, each hydrophone pair measures a phase difference which depends on the wavenumber. These phase differences are given by:

$$
\begin{aligned}
\Delta \phi_{\mathrm{x}} & =\mathrm{k}_{\mathrm{x}} \mathrm{D} \\
\Delta \phi_{\mathrm{y}} & =\mathrm{k}_{\mathrm{y}} \mathrm{D} \\
\Delta \phi_{\mathrm{z}} & =\mathrm{k}_{\mathrm{z}} \mathrm{D} .
\end{aligned}
$$

Once $\mathrm{k}_{\mathrm{X}}, \mathrm{k}_{\mathrm{y}}$, and $\mathrm{k}_{\mathrm{z}}$ are known, the angles $\varphi$ and $\theta$ can be calculated and the directional measurement is complete.

Although three orthogonal hydrophone baselines provide a full description, many commercial USBL systems use an L-shaped array. This array consists of two hydrophone pairs, located along the $\mathrm{x}$ and $\mathrm{y}$ axes of Fig. 2.4. The operation of this array is straightforward if the incident ray is traveling in the $x$-y plane. The vertical angle $\varphi$ is now $90^{\circ}$, so $\mathrm{k}_{\mathrm{z}}=0$ and the unknowns are $\mathrm{k}_{\mathrm{x}}, \mathrm{k}_{\mathrm{y}}$ and $\theta$. If a single hydrophone pair is used to measure one component of the wavenumber, for example $\mathrm{ky}_{\mathrm{y}}$, then the other component can be found from 


$$
\mathrm{k}_{\mathrm{x}}= \pm \sqrt{\mathrm{k}^{2}-\mathrm{k}_{\mathrm{y}}^{2}}
$$

The sign ambiguity, or front-back ambiguity, is resolved by using the measurement from the second hydrophone pair. This is seen in Figure 2.5. Each baseline produces an ambiguous estimate of the bearing angle $\theta$, but by combining their measurements the uncertainty is resolved. As will be shown later, many types of bearing estimation errors for a single baseline will be smaller for incident angles which are nearly perpendicular to the baseline. Thus the estimates from different baselines will typically be weighted unevenly.

The case of a plane wave incident on an L-shaped array from an angle out of the plane of the array is slightly more complicated. If only one baseline is used, there will be only one measurement, and two unknowns - the bearing angle $\theta$ and the declination angle $\varphi$. This results in a cone of constant phase difference, aligned along the baseline (see Fig 2.6). A single baseline cannot discriminate between rays arriving along any direction on the cone. If the relative depths of the source and receiver are known, the angle $\varphi$ can be calculated and it will be possible to solve for $\theta$, although the front-back ambiguity will still be present. If the geometry is not known, but another baseline measuring the other inplane wavenumber component is added, the remaining wavenumber component can be calculated from

$$
k_{z}= \pm \sqrt{k^{2}-k_{y}^{2}-k_{x}^{2}}
$$

Thus a top-bottom ambiguity exists, which is analogous to the front-back ambiguity seen before. Knowledge of the source-receiver geometry (i.e. is the array above or below the source?) can be used to reach a final answer. If the system is mounted on an AUV, the change in estimated bearing caused by a change in vehicle orientation can be used to resolve the ambiguity. For example, if the source is above and behind the vehicle, and the AUV pitches upwards, the apparent bearing should decrease. 


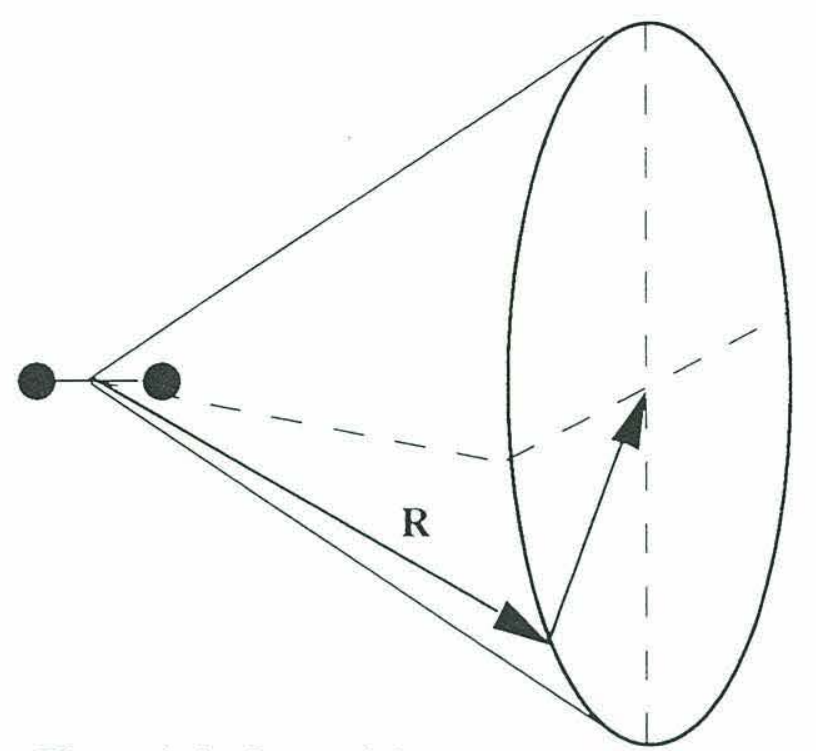

Figure 2.6: Cone of Constant Phase Difference

In Section 1.2 the USBL bearing measurement was introduced and described in terms of finding a time difference $\delta$ t or phase difference $\Delta \phi$. This resulted in the equation for bearing:

$$
\theta=\operatorname{Arcsin}\left(\frac{\Delta \phi \lambda}{2 \pi \mathrm{D}}\right)
$$

It is easy to show that this measurement is equivalent to measuring the $y$-component of the wavenumber. Remembering that $\mathrm{k}=2 \pi / \lambda$, Eq. (2.2), (2.3), and (2.4) above can be combined to give:

$$
\Delta \phi_{\mathrm{y}}=\mathrm{k}_{\mathrm{y}} \mathrm{D}=\left(\frac{2 \pi}{\lambda} \sin (\theta)\right) \mathrm{D},
$$

which can clearly be rewritten to give Eq. (2.7).

The above discussion assumes that there is no ambiguity in the phase difference $\Delta \phi$. For this to be true, the phase difference must be limited to $[-\pi, \pi]$. From Eq.(2.4) it can be seen that the largest possible value for the phase difference along any baseline is:

$$
\Delta \phi_{\max }=\mathrm{kD}=\frac{2 \pi}{\lambda} \mathrm{D}
$$

Setting $\left|\Delta \phi_{\max }\right| \leq \pi$ shows that the designer must set $\mathrm{D} \leq \lambda / 2$. If $\mathrm{D}=\lambda / 2$, equation (2.7) becomes:

$$
\theta=\operatorname{Arcsin}\left(\frac{\Delta \phi}{\pi}\right)
$$


This equation can be checked against Fig. 2.4 to develop a common-sense understanding of the measurement. If the bearing angle $\theta$ is 0 , the wavefront hits both hydrophones in a given pair at once and $\Delta \phi=\delta \mathrm{t}=0$. If the wave impinges from the right at $\theta=\pi$, the signal on the right hydrophone will lead that on the left by $\pi$ and $\delta t=T / 2$. If $\theta=-\pi$, the signal on the right lags by $\pi$ and $\delta t=-T / 2$.

\subsubsection{Hydrophone Array Geometry}

There are several important issues to consider when choosing a geometry for the hydrophone array. The first and most obvious decision is how many hydrophone pairs are needed. If the system will be used for homing in on a beacon, a single hydrophone pair mounted at the nose of the vehicle may suffice. The front-back ambiguity in the bearing angle $\mathrm{q}$ is resolved, as signals from behind will be blocked by the vehicle, and the declination angle may not be required as the vehicle may be programmed to return to a given depth. In most applications, however, an extended array is needed.

It is also possible to exploit different array geometries to improve system performance. One attractive way to improve performance is to increase the distance between sensors, giving a phase or time difference which is larger and therefore easier to measure accurately (in beam-forming terms, increasing the array length to make the array more directive). Simrad has done extensive work developing extended arrays for USBL systems [Storvik, 1983, Vestgaard et al, 1984, Jacobsen et al, 1985]. The Simrad system

uses two hydrophones spaced at less than a half-wavelength, and adds another hydrophone at a spacing of about 2 wavelengths. The larger, aliased, baseline allows a more accurate, but ambiguous, measurement of the phase difference. The small-baseline pair can be used to resolve the ambiguity in bearing. If an accurate model for the spatial correlation of ambient ocean noise is available, it may be possible to position hydrophones to reduce the correlation between channels [Cron and Sherman, 1962].

A number of examples of other hydrophone geometries can be found in the literature. Sonatech has built an target tracking system which uses a ray-trace to determine 
the optimum depth for its acoustic beacon [Watson et al, 1983]. This system has a transducer head with 8 faces, each of which has a 2 element array. The electronics scans through the faces to determine which face is receiving the strongest signal, and tracks the source from face to face. Four-element arrays have been examined, in which the extra baselines are used to improve the estimate through averaging [Farcy \& Leroy, 1979].

\subsubsection{Relationship of USBL Measurement to Conventional Beamforming}

The most common and generally useful technique for processing signals received by sonar arrays is delay-and-sum beamforming. To find the direction of arrival of a wavefront, the signals on all of the hydrophones can be delayed relative to each other to steer the array, then summed to find the magnitude of the signal. The direction giving the largest magnitude is the direction of the wavefront's arrival. For a 2 element USBL system, this is equivalent to guessing different values of $\delta t$ (see Fig. 2.8). If the correct $\delta t$ is guessed, the two waves will interfere constructively when added, giving a large magnitude; incorrect values of $\delta$ t will result in at least partially destructive interference.

Unfortunately, it is impractical to measure bearing by directly estimating time delays for a USBL system. The $26 \mathrm{kHz}$ prototype system built for this thesis can measure phase differences to an accuracy of $0.1^{0}$ in the absence of noise, which corresponds to measuring the time shift with an accuracy of $10 \mathrm{nsec}$. Using beamsteering techniques to estimate bearing with the same accuracy would thus require designing electronics with extremely fine time resolution. Also, knowledge of the transmit frequency is available for the USBL calculation which is not used in the beamsteering approach. Thus the USBL problem deals with phase estimation rather than beamforming.

Monopulse radar techniques have also been adapted for sonar direction-finding. In this technique, the beam is switched between different bearings, and ratios of the received magnitudes are used to estimate bearing. Research into the use of PVDF transducers for this technique appear promising [Henderson, 1987]. 


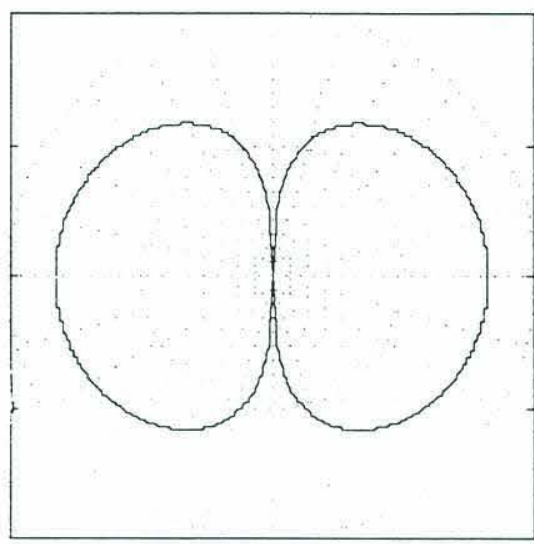

$20 \mathrm{~dB} / \mathrm{div}$

Figure 2.7: Beam Pattern for 2 element unaliased USBL array a) Magnitude of Beam Pattern for 2 element unaliased USBL array

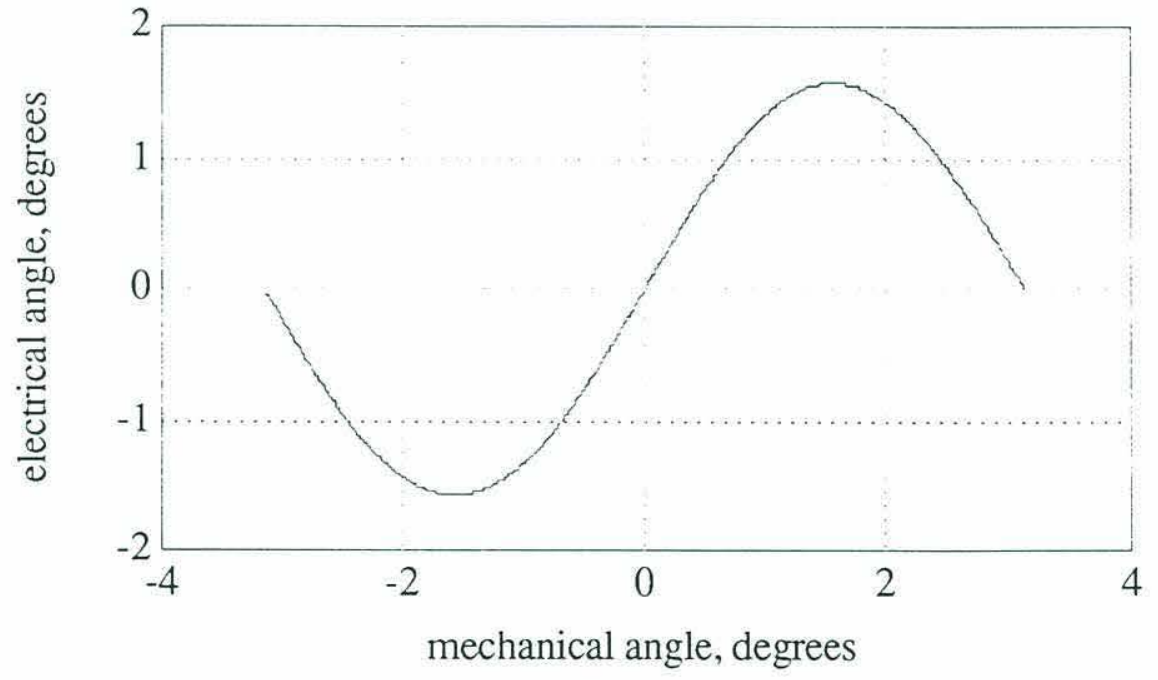

b) Phase of Beam Pattern for 2 element unaliased USBL array 
Figure 2.7a shows the beam pattern for a 2 element, half-wavelength array. The phase plot, Fig. 2.7b, shows the phase pattern for the same array. The front-back ambiguity discussed above is seen, as there are two possible bearings for each phase difference.
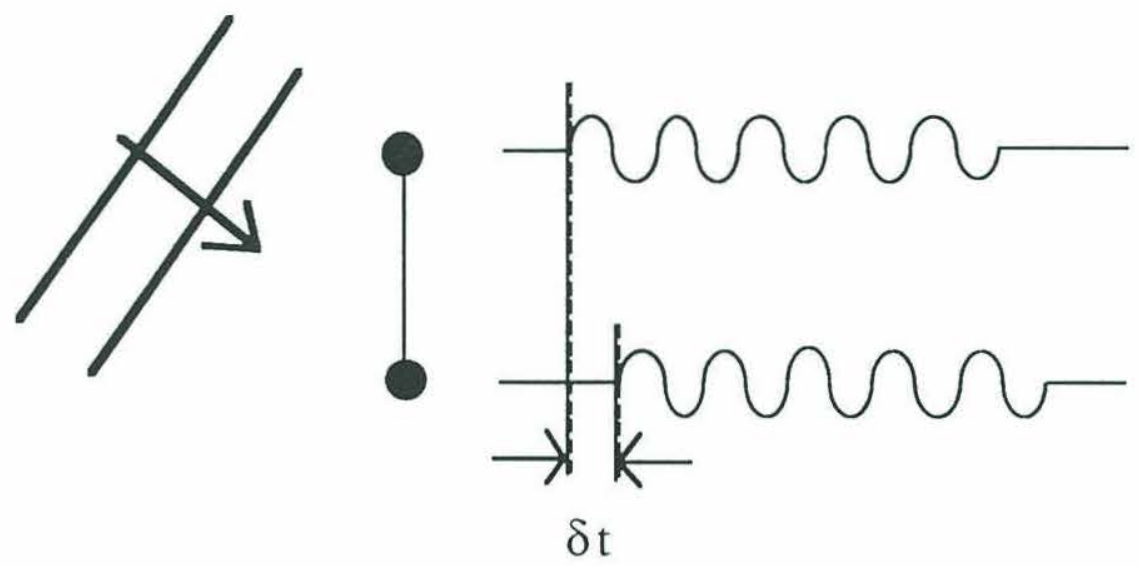

Figure 2.8: Delay-and-Sum Direction Finding for a 2 Element Array

\subsection{Signal Processing for Single-frequency USBL}

In the following sections, the signal processing implementation used for the prototype USBL system is described. Pulse detection, bearing estimation, signal-to-noise estimation, and data filtering are all discussed. The approach taken in this thesis is contrasted with implementations found in commercially available navigation systems.

\subsubsection{Pulse Detection}

In most types of pulse detection, the signals received on the hydrophones are filtered, amplified and compared to a threshold level. If the incoming signal exceeds the threshold, a pulse is detected. The signal must exceed the threshold for a pre-set amount of time in order to be judged to be a valid pulse. The validation period is set to be long 
enough so transient signals from noise spikes or reverberation will not trigger the threshold.

Clearly, the performance of the entire system will depend on the detection threshold chosen. Raising the threshold will decrease the probability of false alarms, but will also decrease the probability of detection (i.e. more valid pulses will be discarded). Standard Bayesian or Neyman-Pearsons hypothesis testing cannot be applied unless an expected signal strength can be predicted [Willsky, Chapter 2]. Since accurately predicting the signal strength is difficult without sophisticated modeling of the environment, the approach taken in testing for this thesis was to determine an appropriate threshold through experimentation.

Since SNR can be estimated on-line, it is possible to use estimated SNR rather than signal power as the detection statistic. The bearing errors at the threshold SNR can be calculated, placing an upper bound on the bearing error. Using SNR as the detection statistic would also help eliminate false triggers from large broad-band noise spikes, and would make possible a detection threshold which does not depend on front-end gain. For the tests described in Chapter 5, however, signal power was used as the detection statistic and SNR data was logged for post-processing.

Calculation of the power at the broadcast frequency for comparison to a detection threshold is straightforward. For reasons which will become clear when the bearing estimation is discussed, the real and imaginary parts of the discrete-time Fourier transform, evaluated at $\omega_{0}$, are calculated. Since the Analog Devices DSP chip is fixed-point and cannot represent any number larger than 1 , the calculation is normalized by the sequence length $\mathrm{M}$ to avoid numerical overflow. The calculated quantities are: 


$$
\begin{aligned}
& X_{\text {real }}\left(\omega_{0}\right)=\frac{1}{M} \sum_{n=\frac{M}{2}}^{\frac{M}{2}-1} x[n] \cos \left(\omega_{0} n\right) \\
& X_{\text {imag }}\left(\omega_{0}\right)=\frac{1}{M} \sum_{n=\frac{M}{2}}^{\frac{M}{2}-1} x[n] \sin \left(\omega_{0} n\right)
\end{aligned}
$$

After these components are known, the (scaled) power at $\omega_{0}$ can be found. This is denoted Ein-band and is given by:

$$
E_{\text {in-band }}=X_{\text {real }^{2}}+X_{\text {imag }^{2}}=\frac{1}{M^{2}}\left|X\left(\omega_{0}\right)\right|^{2} .
$$

This power is then compared to the preset detection threshold to determine if a pulse has arrived.

\subsubsection{Phase Difference Estimate}

As discussed in the introduction, for a single-frequency USBL system the bearing is calculated from an estimate of the phase difference between channels. Two separate approaches to calculating the phase difference are discussed below. The first is the

maximum likelihood estimator (MLE) which was implemented on an Analog Devices DSP chip as part of this thesis. The second is the hard-limited phase estimation approach, used in many commercial USBL systems.

\subsubsection{Maximum Likelihood Estimation of Phase Difference}

A DSP chip can be used to calculate the complex Fourier transform for the signals received on each input channel. The phase of the received signals can be estimated from the real and imaginary parts of the Fourier transform at the broadcast frequency. These phases can be subtracted to form a phase difference. This method can be shown to be the maximum likelihood estimator [Haykin, 1983]. 
This process is now examined in more detail. The first step in estimating the phase difference is to sample the continuous-time signal provided by the analog front-end, thereby creating a discrete sequence $\mathrm{x}[\mathrm{n}]$. The sequence can be transformed according to the discrete time Fourier transform (DTFT), which is defined [Oppenheim, sec. 2.7] by :

$$
\begin{gathered}
x[n]=\frac{1}{2 \pi} \int_{-\pi}^{\pi} X(\omega) \text { ej } \omega n d \omega \\
X(\omega)=\sum_{n=-\infty}^{\infty} x[n] \text { ej } \omega n
\end{gathered}
$$

where $\omega=2 \pi \mathrm{f} / \mathrm{f}_{\text {samp}}$, where fsamp is the sampling frequency. Using Euler's formula, the coefficients $\mathrm{X}(\omega)$ can be split into real and imaginary parts. For the USBL application only one frequency, the broadcast frequency $\omega_{0}$, is of interest. The components of the DTFT evaluated at $\omega_{0}$ are given by

$$
\begin{aligned}
& X_{\text {real }}\left(\omega_{0}\right)=\frac{1}{M} \sum_{n=-\infty}^{\infty} x[n] \cos \left(\omega_{0} n\right) \\
& X_{\text {imag }}\left(\omega_{0}\right)=\frac{1}{M} \sum_{n=-\infty}^{\infty} x[n] \sin \left(\omega_{0} n\right)
\end{aligned}
$$

Since infinitely long summations are difficult to implement, the received signal is broken up into small segments and the phase difference is estimated for each segment. If the summation is over an integer number of sinewave cycles, the orthogonality of the sinusoids summed to create the Fourier series will be maintained. Thus any window can be used, subject to the constraint that the window length $\mathrm{M}$ is an integer number of transmit sinewave cycles.

It can be shown that the rectangular window offers the best performance against noise. Smoother windows are often used in spectral estimation to reduce sidelobe levels, so that the sidelobes from a large peak in the spectrum will not obscure any structure near the peak. As a tradeoff for reducing sidelobe levels, these smoother windows (Hanning, Hamming, etc.) have slightly larger noise bandwidths [Harris, 1978]. In this application, 
however, only the broadcast frequency is of interest, so sidelobe smearing of adjacent frequencies is not a problem.

As the AD2101 does only fixed point arithmetic, the summation must be normalized to prevent overflow. If a rectangular window sequence of length $\mathrm{M}$ is used, the real and imaginary coefficients are given by

$$
\begin{aligned}
& X_{\text {real }}\left(\omega_{0}\right)=\frac{1}{M} \sum_{n=-\frac{M}{2}}^{\frac{M}{2}-1} x[n] \cos \left(\omega_{0} n\right) \\
& X_{\text {imag }}\left(\omega_{0}\right)=\frac{1}{M} \sum_{n=-\frac{M}{2}}^{\frac{M}{2}-1} x[n] \sin \left(\omega_{0} n\right)
\end{aligned}
$$

After the real and imaginary Fourier coefficients are calculated, they can be squared and summed to find the signal power $\mathrm{E}_{\mathrm{in}}$-band. If a pulse is present and $\mathrm{E}_{\mathrm{in} \text {-band }}$ exceeds the detection threshold, the phase on each channel can be calculated from

$$
\phi=\operatorname{Arctan}\left(\frac{X_{\text {imag }}}{X_{\text {real }}}\right) \text {. }
$$

The phase difference

$$
\Delta \phi=\phi_{1}-\phi_{2}
$$

can then be found; care must be taken with the sign conventions to avoid answers larger than $2 \pi$. The bearing angle $\theta$ can now be calculated, as discussed above.

The choice of window length will have a significant impact on system performance, for a number of reasons. The first is that windowing in time results in filtering in frequency. The shape of the filtering is given by the Fourier transform of the window used. For the rectangular window this is [Siebert, 1986]:

$$
W(f)=\frac{\sin \left[\pi T\left(f-f_{o}\right)\right]}{\pi T\left(f-f_{o}\right)}
$$

This leads to a reduction in noise bandwidth. Harris gives the noise bandwidth of the rectangular window as approximately

$$
\mathrm{BW}=\frac{1}{\mathrm{~T}_{\mathrm{w}}},
$$


where $T_{\mathrm{W}}$ is the window duration. This digital filtering must be taken into account when calculating the effects of ambient and front-end noise.

The window length chosen will also determine the susceptibility of the bearing calculation to errors caused by multipath interference. Generally a short window length should be used, so that at least a few windows will contain uncontaminated phase information from a non-overlapped arrival. For the tests described in Chapters $4 \& 5$ a window length of 4 cycles of the signal frequency was used. In this implementation bearing estimates taken throughout the entire pulse are recorded, and decisions are then made as to which, if any, windows contain good data. This is in contrast to commercial systems, which generally base their estimate on a preset section of the pulse and throw out the rest.

Perhaps because of the similarity in their names, it is easy to confuse the DiscreteTime Fourier Transform (DTFT) with the Discrete Fourier Transform (DFT). The DFT is derived from the DTFT be assuming that the sampled signal is periodic in time. This has the effect of discretizing the spectrum. The advantage of doing this is that the calculations can be greatly speeded up by using the FFT algorithm. However, for the USBL application it is only necessary to calculate the Fourier coefficients at one frequency, and are not the overall shape of the spectrum. Thus the DTFT approach is more appropriate for this application than the DFT-FFT approach.

\subsubsection{Phase Difference Estimation after Signal Hard-limiting}

Since most USBL systems are developed as commercial products by companies, the available literature tends to be descriptive and not extremely detailed. Still, it is possible to form a reasonable picture of the processing techniques used. It seems clear that most commercial systems use a signal hard-limiting approach for phase difference estimation similar to that described by Russell Light [Light, 1988]. In this implementation, signals from each hydrophone are amplified, run through a fairly wide bandpass filter, and then 
hardlimited. The signals then pass through a narrow bandpass filter centered at the broadcast frequency, and are compared to a threshold to determine if a pulse is present. The hardlimiting ensures that all signals have the same power before the final filter stage, so there is no need to change the detection threshold as the range increases and signal strength drops. The signals are then exclusive-OR'ed, which as shown below produces a signal whose duty cycle is proportional to the phase difference. The system assumes that if there are multiple arrivals, the first arrival will be the direct path, and other arrivals are ignored. The bearing measurement is made using only 8 cycles of the sine wave taken right after the initial transients have died away. This assumes that any interference from overlapped multipaths will occur later in the pulse.

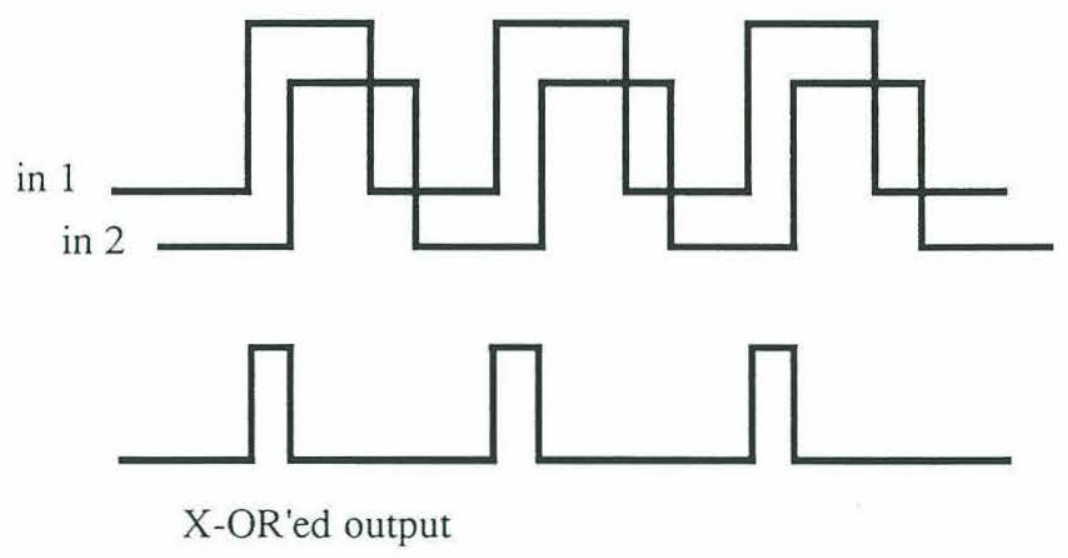

Figure 2.9: Phase Difference Estimation Using Exclusive-OR

There are a number of commercially available USBL systems which operate on this basic principle, though with some interesting additions. Ferranti O.R.E. sells a Trackpoint II system which has a large share of the market. This system seems to be similar to Russell Light's, using an exclusive-OR and $30 \mathrm{MHz}$ clock to achieve $0.3^{\circ}$ phase accuracy in the absence of noise. As in Light's system, only a small amount of the available pulse is used, in order to minimize multipath interference. Simrad offers a system which averages 
6 phase difference estimates taken near the start of the pulse and has similar performance claims. A performance model for hard-limiting systems is presented in the next chapter and compared with the MLE method.

\subsubsection{Calculation of Power and On-line Signal-to-Noise Estimate}

In section 2.2.1 the calculation of the signal power, or in-band power, was

discussed. This quantity is compared to the detection threshold to determine if a pulse is

received. At the same time the total power in the received signal, also called the wide-band power, is calculated. If a pulse is detected, the narrowband power will basically be the signal power $\mathrm{S}$, and the wide-band power will be $\mathrm{S}+\mathrm{N}$, where $\mathrm{N}$ is the noise power over the whole band. For the current implementation, the quantities $\mathrm{S}+\mathrm{N}$ and $\mathrm{S}$ were simply logged for post-processing. However, these data can be manipulated to form a SNR estimate on-line. This estimate can be used to estimate the confidence which should be placed in the bearing output.

Since the calculation of the signal power has already been described, it remains only to describe calculation of the wide-band, or total, power. The total (or "wideband") power in the digitized signal is given by [Oppenheim, sec 2.7]:

$$
E_{\text {wideband }}=\frac{1}{M} \sum_{n=0}^{M-1}|x[n]|^{2}
$$

In Appendix B, the scaling factors relating $E_{\text {in-band }}$ and $E_{\text {wideband }}$ are calculated. It is found that if a signal is present, with no noise, they are related by

$$
\mathrm{E}_{\text {wideband }}=\frac{2 \mathrm{M}}{\mathrm{M}+1} \mathrm{E}_{\text {in-band }} \text {. }
$$

Errors resulting from fixed-point arithmetic will keep the equality from being exact. The SNR can then be estimated by

$$
S N R=\frac{\frac{2 M}{M+1} E_{i n-b a n d}}{\left(E_{\text {wideband }}-\frac{2 M}{M+1} E_{\text {in-band }}\right)}
$$




\subsection{Filtering of Data}

Any useful USBL system must be capable of filtering the data it receives. At a minimum, filtering must be provided to rejection of short-lived noise spikes which may trigger the detection threshold. Provisions must be made to reject data which is contaminated by multipath interference. An on-line estimate of SNR can be used to generate an estimate of errors caused by noise, which can be used by a filtering routine. One of the main objectives in testing the prototype USBL system was to learn what types of filtering might be used to improve system performance. Suggestions for data filtering will be found in discussion of the in-water tests results.

Useful hints about data filtering techniques can be found from studying the available USBL literature. Many companies, such as Ferranti, Simrad, Sonatech, and EDO offer systems which seem to be similar. All of these systems filter the output of the USBL to reject "outliers" - results which disagree with previous positions by an unreasonable amount. Some use a Kalman filter to track the vehicle's location. This tracking allows continued estimation of the vehicle's position in situations where the system receives only bad returns for some time. Most of these systems do not use raytracing to improve performance, although the Sonatech system uses ray-tracing to determine advantageous relative positionings of the source and receiver [Watson et al, 1983]. The Simrad USBL system calibrates itself to find the average sound speed [Storvik, verbal communication, July 91].

As mentioned above, many hard-limiting USBL systems use only a small segment near the beginning of the received pulse for the bearing estimation. This is done as it is assumed that multipath interference is always present. When the hydrophone array is close to a strongly reflecting surface such as a ship hull or the water surface, as is true for shipmounted systems, this may be a reasonable assumption. In other situations, such as a USBL mounted on a submersible operating in deep water, this assumption will not be true 
and would result in the rejection of large amounts of good data. A good understanding of the acoustic environment could be combined with the computational flexibility of the DSP chip to produce a system capable of changing its data filtering rules depending on the situation. 


\section{Error Analysis}

\subsection{Introduction}

In this chapter, sources of error in the USBL implementation described above are identified and evaluated. Where possible, ways to minimize the errors are suggested. Special attention is paid to the effects of noise. A model for bearing estimation performance in the presence of noise is presented, and compared with laboratory measurements. Sources of noise in the prototype USBL system are identified, and predictions of bearing estimation errors vs. range are presented.

\subsection{Framework for Linearized Error Analysis}

Useful error predictions can be made by analyzing the basic USBL equation. As will be remembered from Section 1.2, the basic USBL equation is

$$
\theta=\operatorname{Arcsin}\left(\frac{\Delta \phi \lambda}{2 \pi \mathrm{D}}\right)
$$

This equation can be rewritten slightly so that it can be seen that the bearing angle $\mathrm{q}$ depends on the measured phase difference, sound speed, and baseline length, giving:

$$
\theta=F(\Delta \phi, D, c)=\operatorname{Arcsin}\left(\frac{\Delta \phi c}{2 \pi f_{0} D}\right) .
$$

This expression can now be expanded in a Taylor series. If the expansion is made about the measured values for $\Delta \phi, D$, and $\mathrm{c}$, the effects of errors in these measurements can be seen. It can generally be assumed that the errors will be a small fraction of the actual value, so the expansion can be linearized. This yields Eq. (3.3).

$$
\begin{gathered}
\theta=\theta_{0}+\theta_{\text {err }}=\mathrm{F}\left(\Delta \phi_{0}, \mathrm{D}_{0}, \mathrm{c}_{0}\right)+ \\
\varepsilon_{\Delta \phi} \frac{\delta}{\delta \Delta \phi} \mathrm{F}\left(\Delta \phi_{0}, \mathrm{D}_{0}, \mathrm{c}_{0}\right)+\varepsilon_{\mathrm{c}} \frac{\delta}{\delta \mathrm{c}} \mathrm{F}\left(\Delta \phi_{0}, \mathrm{D}_{0}, \mathrm{c}_{0}\right)+\varepsilon_{\mathrm{D}} \frac{\delta}{\delta \mathrm{D}} \mathrm{F}\left(\Delta \phi_{0}, \mathrm{D}_{0}, \mathrm{c}_{0}\right)
\end{gathered}
$$

The terms due to errors in phase difference, sound velocity, and baseline length are evaluated in the sections below. It is important to note that the Taylor series expansion 
does not provide a good framework for analyzing errors due to Doppler shifts. A signal frequency is assumed for calculation of the phase difference, and the errors caused by assuming an incorrect frequency cannot be easily linearized. Doppler errors are therefore treated separately in section 3.1.4.

\subsubsection{Bearing Errors caused by Uncertainty in Time/Phase Difference Estimate}

Equation (3.1) above shows that the sensitivity of the USBL system is governed by the shape of the Arcsin function. The Arcsin has its steepest slope for small values of the argument, when $\Delta \phi$ is small and signals are incident broadside on the array. For these directions a small change in $\Delta \phi$ yields a noticeable change in bearing. The calculation is much less sensitive to changes in bearing for large values of $\Delta \phi$, when the array is operating close to endfire.

This dependence of sensitivity on bearing angle is seen in the results of the Taylor series expansion. The linearized error term from Eq. (3.3) yields

$$
\theta_{\text {err, } \Delta \phi}=\frac{\varepsilon_{\Delta 0} c}{\left(2 \pi \mathrm{f}_{0} \mathrm{D}\right) \sqrt{1-\left(\frac{\Delta \phi c}{2 \pi \mathrm{f}_{0} \mathrm{D}}\right)^{2}}}
$$

Figure 3.10 shows the expected errors in bearing over the full range of bearings assuming per $1^{0}$ error in $\Delta \phi$. It can be immediately seen that for an L-shaped array or other multiplebaseline array, it is advantageous to weight estimates from baselines which report a small bearing error more heavily.

\subsubsection{Bearing Errors caused by Errors in Assumed Sound Speed}

During operation the vehicle will have temperature, salinity, and depth sensors which allow it to estimate the sound speed. An analysis of the errors introduced by assuming a false value for the speed of sound will help the designer decide how sensitive the vehicle's sensors need to be to support acoustic navigation. It should be noted that for the direct USBL measurement only the sound speed at the hydrophones must be known, 


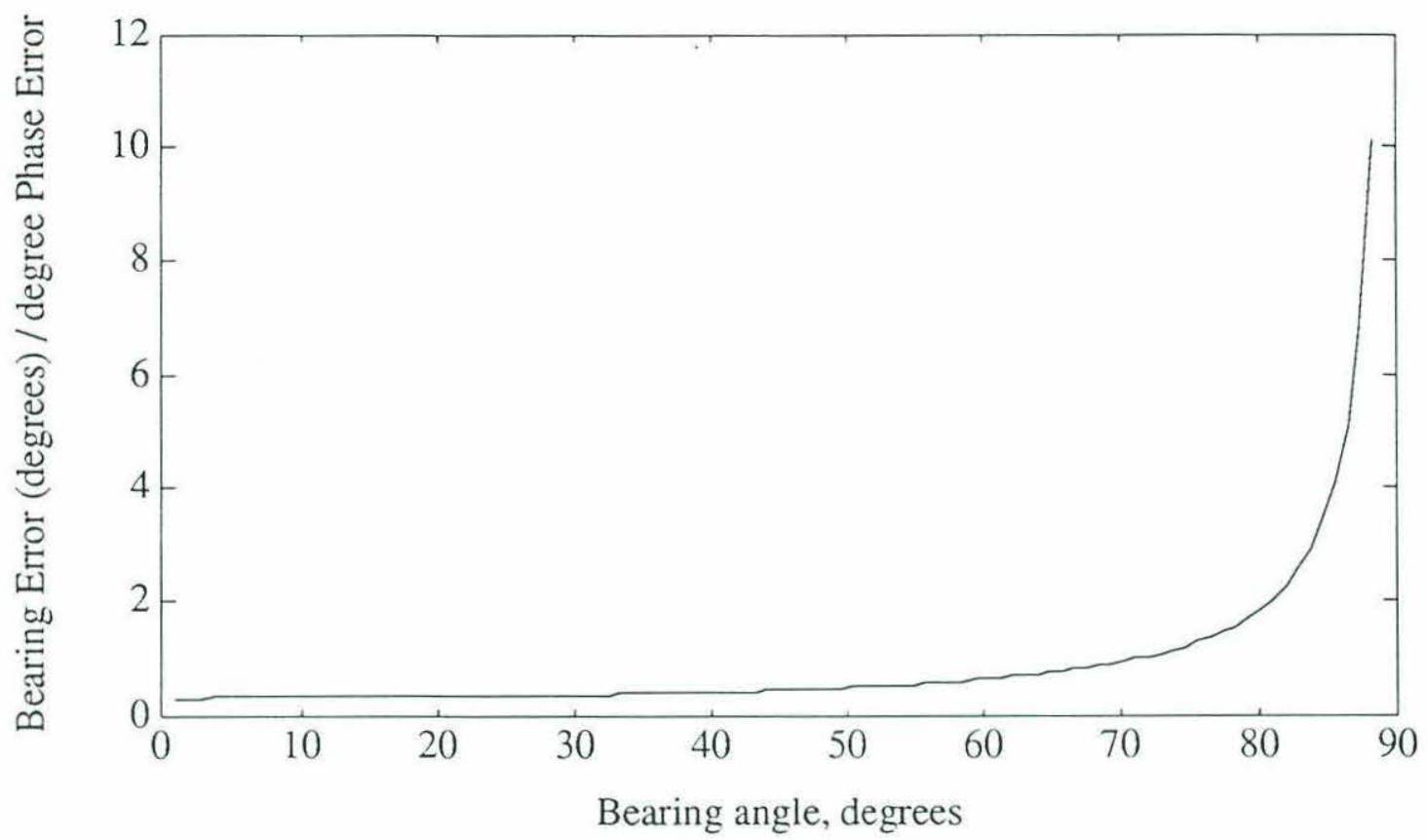

Figure 3.10: Bearing Error Resulting from Error in Phase Difference

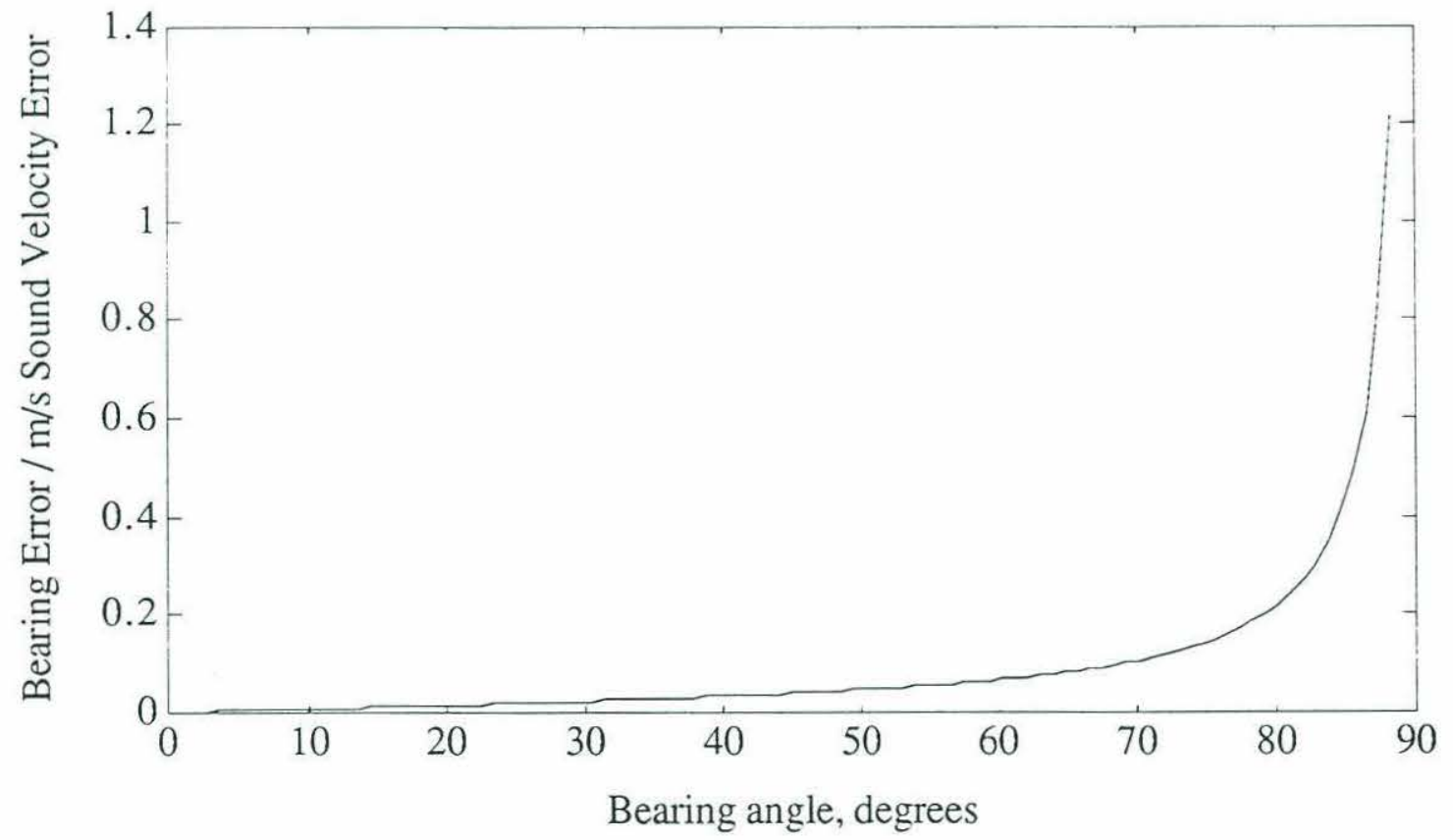

Figure 3.11: Bearing Errors Resulting from Errors in Sound Speed Estimate 
although a sound speed profile vs. depth is needed if the system will correct for refraction and multipath effects.

The Taylor series expansion term above corresponding to errors in assumed sound speed can be shown to be equal to

$$
\theta_{\text {err, } c}=\frac{\varepsilon_{\mathrm{c}} \Delta \phi}{\left(2 \pi \mathrm{f}_{0} \mathrm{D}\right) \sqrt{1-\left(\frac{\Delta \phi c}{2 \pi \mathrm{f}_{0} \mathrm{D}}\right)^{2}}} .
$$

Figure 3.11 shows the error introduced per $1 \mathrm{~m} / \mathrm{s}$ error in estimated speed sound. For small bearing angles the error is fairly small, but it increases with bearing angle. This plot has implications for USBL system design. If an multi-baseline array is used and the sound speed is not known with sufficient confidence, the weighting of bearing measurements from the different baselines should take into account the possible errors from a bad sound speed estimate. In other words, estimates with small bearing angles might be weighted even more heavily than suggested in section 3.1.

\subsubsection{Bearing Errors caused by Uncertainty in Hydrophone Position}

Misalignment of the hydrophone pair.can be a serious source of errors in the bearing estimation. Even in manufacturing operations it can be difficult to place the sensors accurately, so a calibration of the array is often required. Ferranti O.R.E., maker of the Trackpoint II system, has developed a hydrophone array calibration system which gives significant increases in performance - from a 2-30 bearing error to $0.3^{\circ}$ error, in tests without noise [Byron Sellman, verbal communication, July 91].

A framework for analysis of positioning errors is seen in Fig. 3.14. The position of one of the hydrophones is assumed to be known exactly, while the position of the second can vary from the intended position by an $\mathrm{x}$ error of $\varepsilon_{\mathrm{x}}$ and a $\mathrm{y}$ error of $\varepsilon_{\mathrm{y}}$. As seen below, an error in the $\mathrm{x}$ direction introduces an error in the baseline length $\mathrm{D}$. This error can be calculated by evaluating the Taylor series error term from Eq. (3.3), yielding 


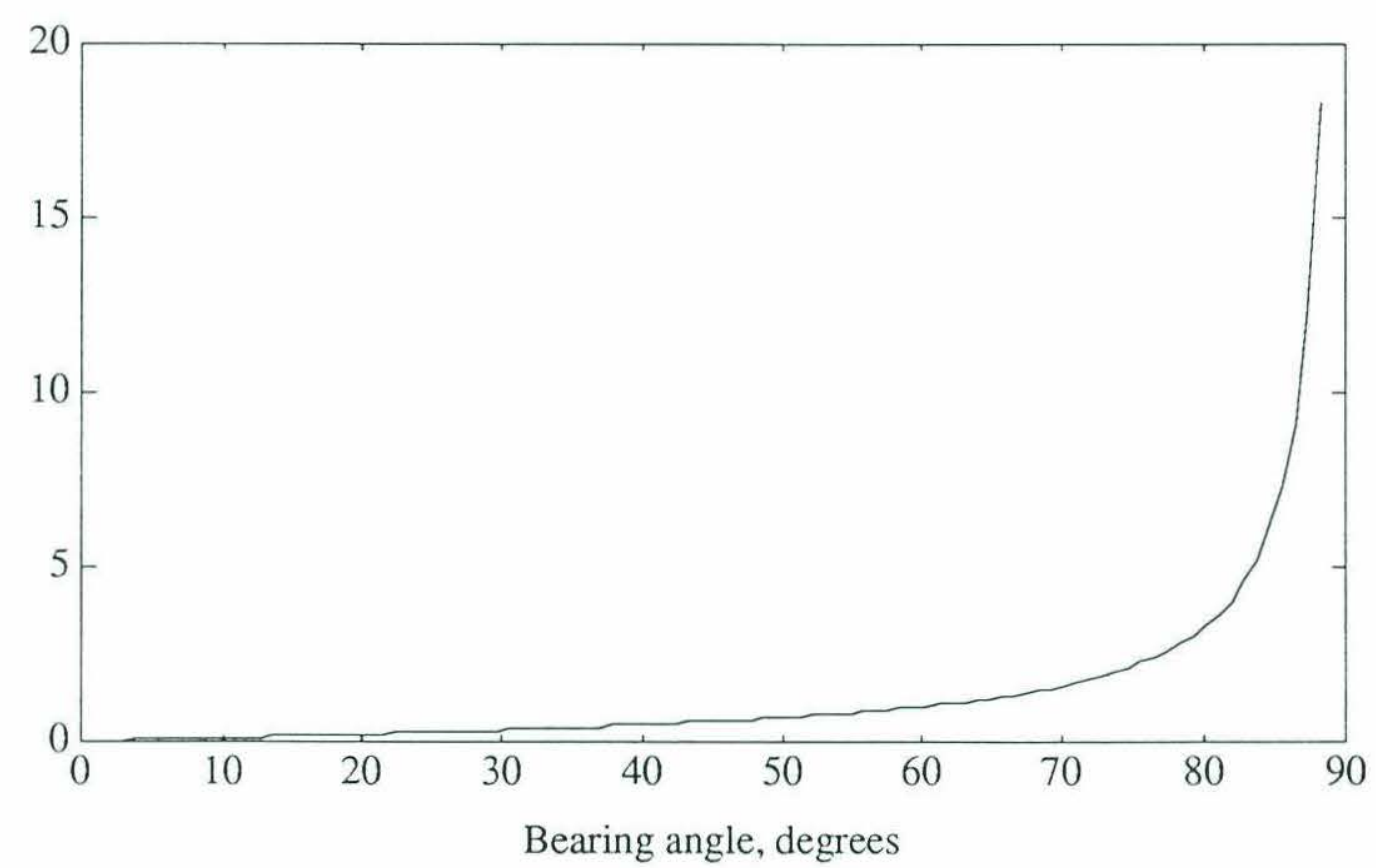

Figure 3.12: Bearing Errors Resulting from Errors in Baseline Length (X-direction)

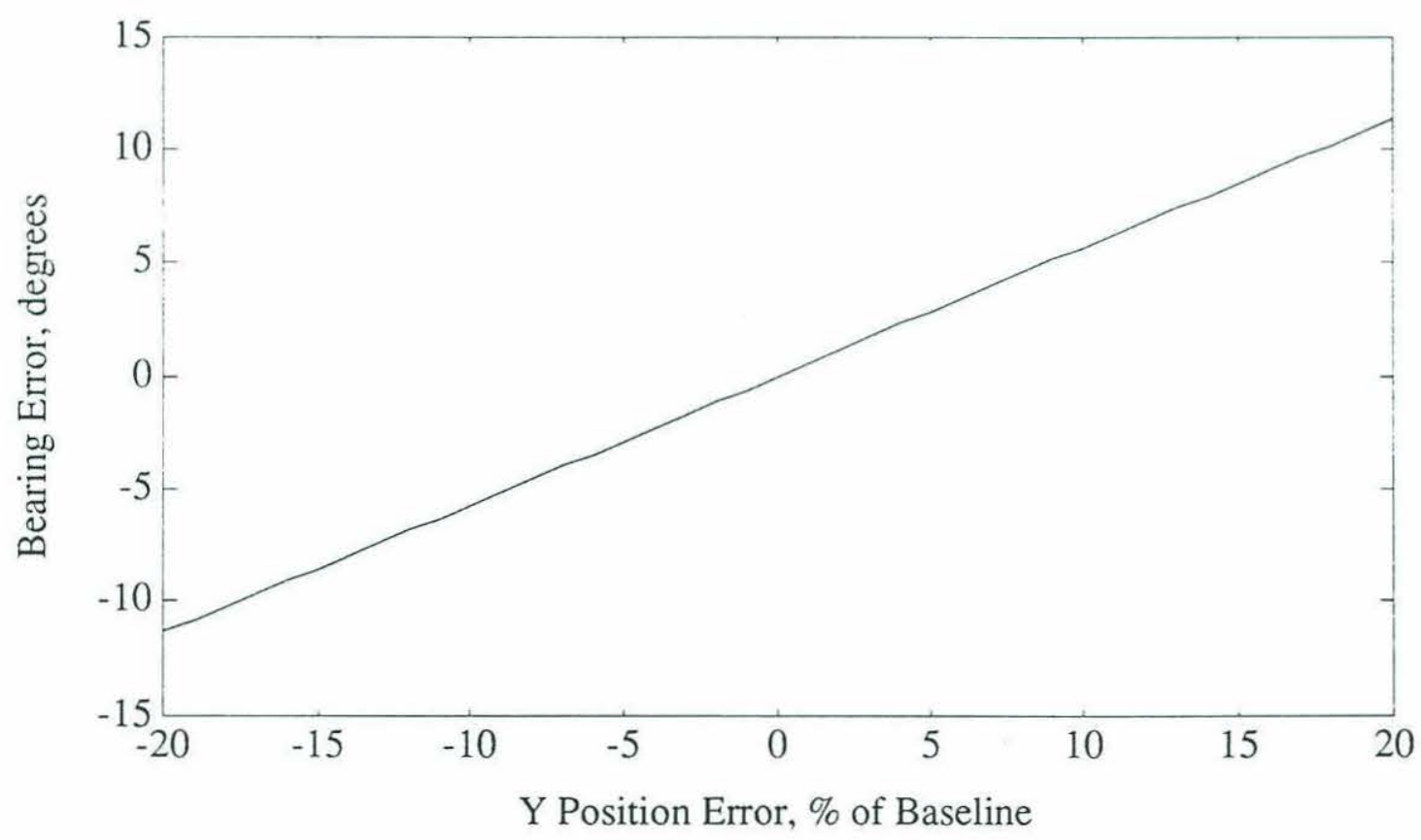

Figure 3.13: Bearing Error Resulting from Hydrophone Offset in $Y$ 


$$
\theta_{\text {err. } D}=\frac{-\varepsilon_{D} \Delta \phi c}{\left(2 \pi f_{0} D^{2}\right) \sqrt{1-\left(\frac{\Delta \varphi c}{2 \pi f_{0} D}\right)^{2}}}
$$

An error in the y direction will introduce an offset in angle directly:

$$
\theta_{\text {offset }}=\arctan \left(\frac{\varepsilon_{\mathrm{y}}}{D_{\mathrm{x}}}\right)
$$

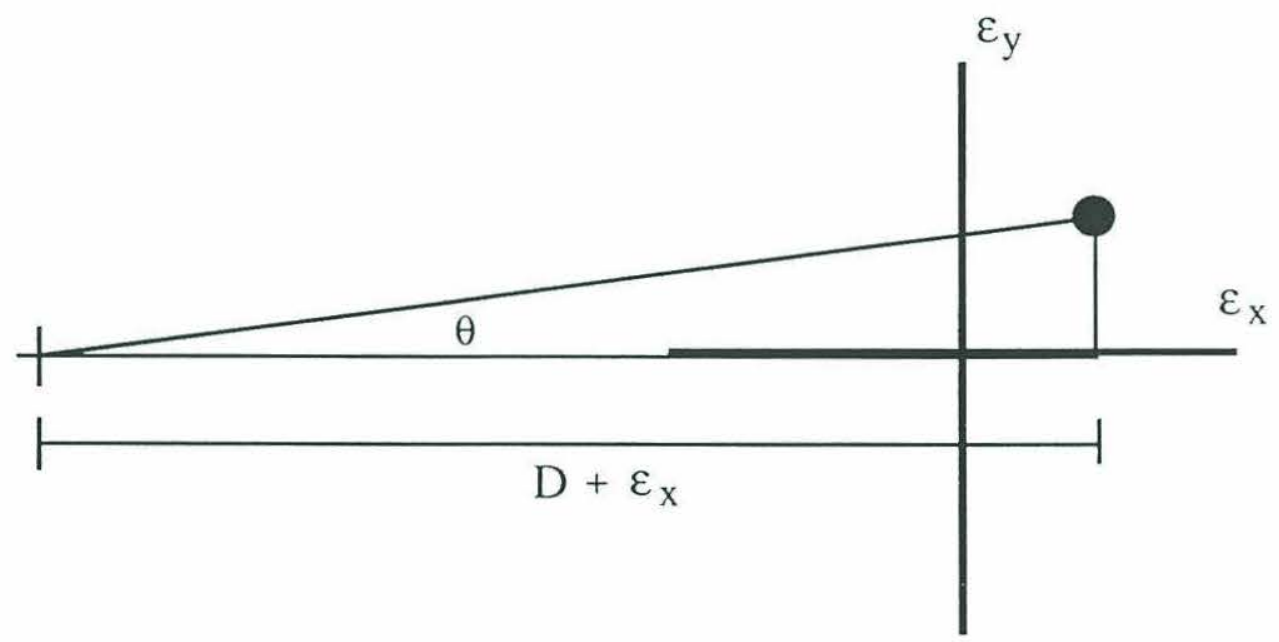

Figure 3.14: Hydrophone Position Coordinate System

Errors due to displacement Figures 3.12 and 3.13 show the errors resulting from displacement in $\mathrm{x}$ and $\mathrm{y}$. The results shown are for a $26 \mathrm{kHz}$ system. It is seen that quite large errors can result. Eventually, as the hydrophones are moved farther apart, the phase difference between the received signals will exceed $\pi$ and aliasing will be seen.

\subsubsection{Doppler/Detuning Errors}

Bearing estimate errors will result when the frequency received by the USBL system differs from the nominal broadcast frequency. This situation arises when a Doppler shift is present due to vehicle motion, or when the pinger used is not tuned accurately to its nominal frequency. Doppler shifts due to vehicle motion can be easily estimated; for 
example, a vehicle moving will perceive a $26 \mathrm{~Hz}$ shift in a $26 \mathrm{kHz}$ tone when moving away from a source at 3 knots, and a $53 \mathrm{~Hz}$ shift when moving at 6 knots. Frequency errors due to detuning will often be larger in magnitude. In the dockside testing described above the frequency of the pinger was measured to be $25.7 \mathrm{kHz}$, instead of the $26 \mathrm{kHz}$ expected. Problems with pinger detuning can be overcome either by changing the frequency of the stored sine and cosine segments in the DSP program (as was done for the dockside tests), or by retuning the pinger. In either case, it is helpful to have some idea of the errors introduced by frequency shift.

Two approaches were taken to evaluated the magnitude of Doppler errors. An attempt was made to find an simple closed-form expression for phase difference errors due to Doppler shifts. This proved difficult, and instead the calculations involved were done numerically. First the phase on each channel was calculated, as a function of the Doppler error $\delta \omega$ and phase shift $\phi$. In the actual system the phase calculation is of course done by summation, but to make this calculation easier the summations were replaced by integrations. The calculated quantities were:

$$
\begin{gathered}
\phi_{1}=\operatorname{Arctan}\left(\frac{\int_{0}^{\tau} \sin \left(\left(\omega_{0}+\delta \omega\right) t\right) \sin \left(\omega_{0} t\right) d t}{\int_{0}^{\tau} \sin \left(\left(\omega_{0}+\delta \omega\right) t\right) \cos \left(\omega_{0} t\right) d t}\right) \\
\phi_{2}=\operatorname{Arctan}\left(\frac{\int_{0}^{\tau} \sin \left(\left(\omega_{0}+\delta \omega\right) t+\phi\right) \sin \left(\omega_{0} t\right) d t}{\int_{0}^{\tau} \sin \left(\left(\omega_{0}+\delta \omega\right) t+\phi\right) \cos \left(\omega_{0} t\right) d t}\right) \\
\sigma(\delta \omega)=\frac{1}{2 \pi} \int_{0}^{2 \pi}\left[\left(\phi_{1}(\delta \omega)-\phi_{2}(\delta \omega)\right)-\left(\phi_{1}(\delta \omega=0)-\phi_{2}(\delta \omega=0)\right)\right] d \omega
\end{gathered}
$$

The phase difference was then calculated, and results were averaged over $\phi$ for each fixed value of $\delta \omega$. 


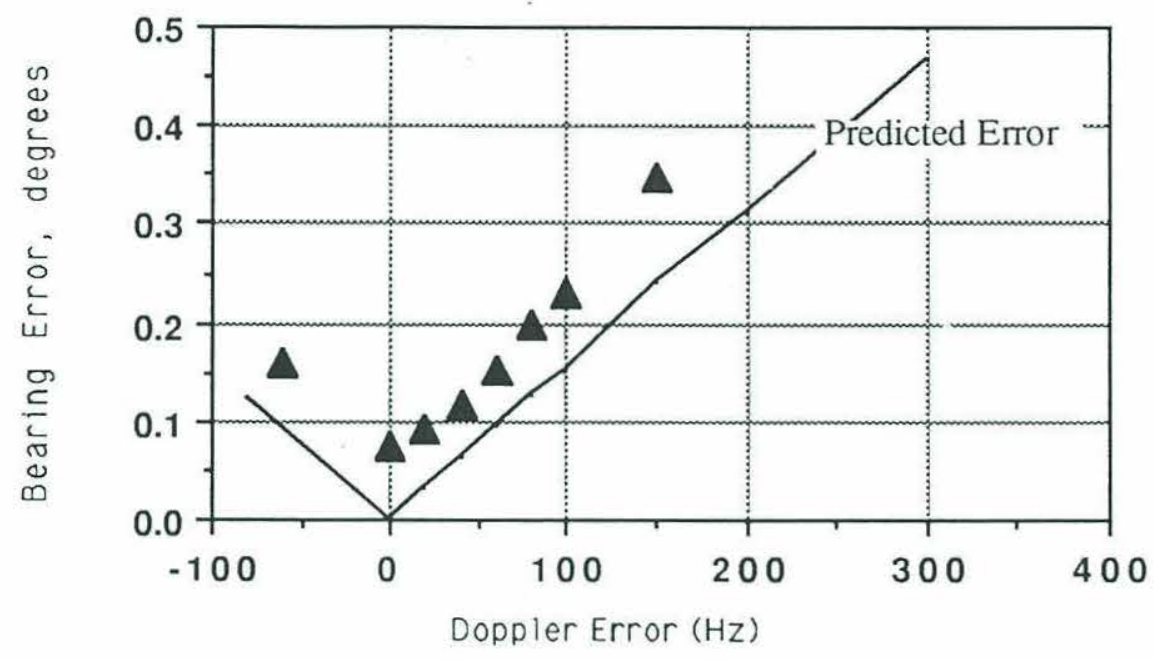

Figure 3.15: Measured vs. Calculated Doppler Error diamonds $=$ measured errors $\mathrm{w} / 13$ cycle integration time 4 and 13 cycle integration period results identical, shown as line

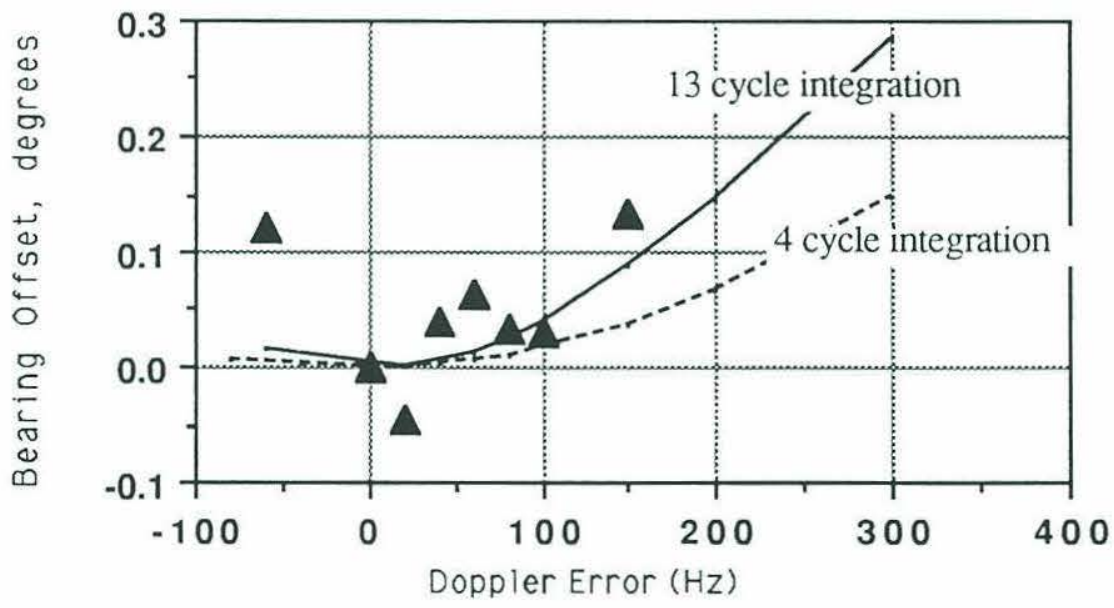

Figure 3.16: Calculated and Measured Offsets due to Doppler diamonds $=$ measured offsets $\mathrm{w} / 13$ cycle integration time 4 and 13 cycle integration period results shown 
These numerical results were checked against measurements done in lab with a carefully detuned signal generator. These measurements were done with a DSP processing window length of $0.5 \mathrm{msec}$, or 13 cycles of a $26 \mathrm{kHz}$ sinewave. Figure 3.15 compares the experimental measurements with results of the calculation. As can be seen, there is a nearly constant offset between the measured and calculated values, representing an error which is presumably due to fixed-point calculation roundoff and A/D quantization noise. This plot strongly suggests that the numerical calculation model Doppler errors accurately. Results of calculations for the $25.7 \mathrm{kHz}$ frequency, 4 cycle window length implementation used in the dockside tests are shown, and it is seen that the results for estimated error of the phase difference are almost identical. The calculated results predict a small DC offset which depends on the amount of Doppler shift. In Figure 3.16 the phase difference offset errors due to Doppler for the three situations are shown. The results here are more ambiguous, although it is clear that for small Doppler shifts the DC offset of the measured values is lost in noise.

\subsection{Model of Performance in the Presence of Noise}

The sources of error discussed above do not depend on the type of signal processing used to implement the USBL system. In order to compare different processing schemes, it is important to be able to characterize the performance of the processing in the presence of real-world problems such as noise and multipath.

The expected performance for the prototype USBL system in the presence of noise can be calculated using a simple model. This model makes a number of simplifying assumptions, which will be stated below. The most important assumption is that the noise present on different sensors will be uncorrelated, additive white Gaussian noise. Ambient ocean noise will of course be generally correlated spatially and temporally [Kuperman, 1980], but the assumption holds more true for electronics noise [Horowitz and Hill, 1980], which is a larger contributor to the overall noise in the system. 
The model is a simple geometric one, which represents the received signals as vectors in the complex plane. In Fig. 3.17 the axes plotted are the real and imaginary parts of the Fourier transform. The length of each arrow displays the magnitude of the signal, and its angle gives its phase. The true phase difference is the angle between the two vectors. A noise voltage is added to the signal, denoted by the smaller arrow added to the tip of the signal vector. The resultant vector is that recorded by the sensors, and the angle between two such resultant vectors will be the phase difference estimate calculated by the USBL system.

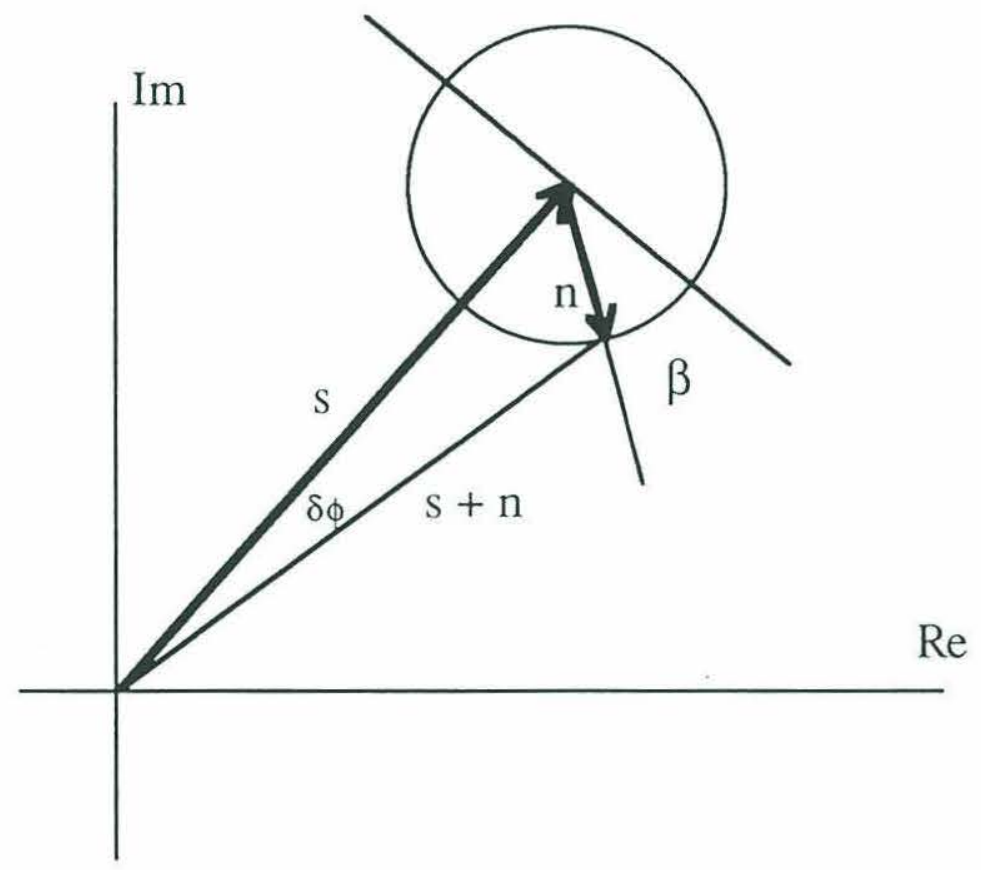

Figure 3.17: Geometric Model for Effects of Noise on One Channel 
Simple geometry can now be used to estimate the errors caused by the noise. The error for a given noise vector is given by

$$
\delta \Phi=\operatorname{Arctan}\left(\frac{\mathrm{n} \cos (\beta)}{\mathrm{s}-\mathrm{n} \sin (\beta)}\right),
$$

where $\mathrm{n}$ is the magnitude of the noise vector and $\mathrm{s}$ is the magnitude of the signal vector. If the signal-to-noise ratio is high enough (10 dB or more), then the value of the Arctan is well approximated by its argument and

$$
\delta \Phi=\frac{\mathrm{n} \cos (\beta)}{\mathrm{s}}
$$

The standard deviation for one channel can be found from the root-mean-square of the error:

$$
\sigma_{1 \text { channel }}=\sqrt{\left\langle\left(\frac{\mathrm{n} \cos (\beta)}{\mathrm{s}}\right)^{2}\right\rangle}=\frac{\mathrm{n}}{\sqrt{2} \mathrm{~s}} .
$$

where the averaging is carried out over $\beta=[0,2 \pi]$. This result gives the error for one channel only. Assuming that the errors on different channels are independent (i.e. the noise is not spatially correlated) means that variances will be added when the results from the two channels are subtracted to find a phase difference. Thus the standard deviation for the phase difference estimate is

$$
\sigma_{\Delta \Phi}=\sqrt{2} \sigma_{1 \text { channel }}=\frac{\mathrm{n}}{\mathrm{s}} .
$$

The quantities $s$ and $\mathrm{n}$ above represent the RMS values of the signal and noise voltages. If the error is rewritten in term of the SNR (power), it becomes

$$
\sigma_{\Delta \Phi}=\frac{1}{\sqrt{\mathrm{SNR}}}
$$

This model is clearly an oversimplification, but it can be useful.

Benchtop tests of the USBL system were carried out to test the accuracy of the phase difference calculation. Details of the electronics used are available in Appendix A. A precisely tuned sinewave was generated and split into two buffered channels, one of which was phase-shifted relative to the other. These signals were used as input to the DSP system, and phase differences could be measured with an standard deviation of $0.1^{0}$. Two independent noise generators were constructed [Graf, 1983] and the noise amplitudes were tuned to be equal. The signal and noise voltages were added with summing amplifiers, 
which could be tuned to yield different signal-to-noise ratios. The signals were then input to the DSP system, and the phase difference calculation was carried out using a window length of 13 cycles.

The results of these tests are displayed in Fig 3.18, along with the predictions of the model. The measured errors are agree well with predictions at high SNR, but agree less well at lower SNR. The reasons for this are not entirely clear. One possible explanation is that the noise source used probably does not produce white Gaussian noise, as was assumed in the model. A more likely source of error is the difficulty, especially at lower SNR, of accurately reading peak-to-peak noise voltages from the oscilloscope. An estimate of the experimenter's ability to read noise levels is included on the plot. Consistently conservative estimates of the noise voltage at lower SNR could lead to the discrepancies observed, although the size of the offset was surprising.

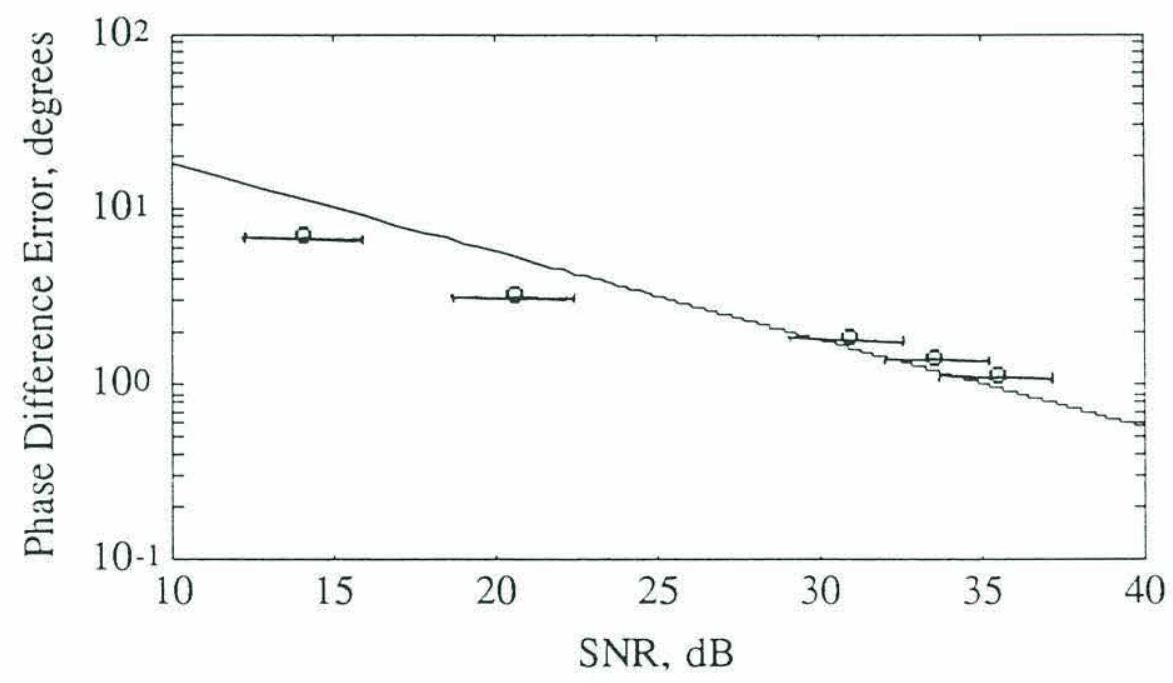

Figure 3.18: Experiment vs. Model Predictions for Noise Performance

\subsection{Comparison with Hard-limited USBL Systems}

The Sea Grant AUV Lab is developing a hard-limited USBL system based on Russell Light's work. David Chen, an undergraduate working at Sea Grant, built a hardlimited system and evaluated its performance [Chen, 1992]. Part of his work included modeling the effects of noise. The hard-limited system measures zero-crossings of the 
received waves, which contain the same phase information which is used for the bearing estimation algorithm described above. When adjustments for units are made, it can be shown that the model developed for the hard-limited system makes error predictions identical to those shown for the MLE system. This comparison has several implications. First, it is a useful check for both models, since different mathematical approaches were used for each. Second, it indicates that calculating the phase difference using the DSP algorithm described will not lead to any large performance gains. Advantages over more conventional hard-limited systems include ease of reconfiguring the system (for example changing pinger frequency), the availability of an on-line SNR ratio, and the ability to implement more flexible filtering to identify the onset of multipath interference.

\subsection{Sources of Noise}

The main identifiable sources of noise, listed in order of strength, are electronics noise, ambient ocean noise, and quantization noise. Since these sources are independent, it is safe to assume that the noise signals are uncorrelated. Their powers may be added to find the total noise power:

$$
\sigma^{2} \text { noise }=\sigma^{2} \text { frontend }+\sigma^{2} \text { ambient }+\sigma^{2} \text { quantization }
$$

The three sections which follow examine each of these noise sources and arrive at estimates of their contributions.

\subsubsection{Analog Circuitry Noise}

In designing the analog front end for the USBL system, filters and preamplifiers with low noise levels were chosen in order to maximize signal-to-noise level. The electronics specifications are discussed in Chapter 4, and in detail in Appendix A.

The front-end electronics consist of preamplifiers, filters, and amplifiers. In Table 3.1 the manufacturer's noise specifications are presented, scaled for a $20 \mathrm{kHz}$ bandpass filter bandwidth. In-lab measurements of the noise levels at each point in the circuit are 
also presented. To take these measurements the inputs of the preamplifiers were shorted together, and the noise signals from various points in the system were measured on an oscilloscope after being amplified by an Ithaco 1201 low noise amplifier. A $3 \mathrm{kHz}-100$ $\mathrm{kHz}$ bandwidth was used for the measurement. As the noise levels for the two channels were comparable, the results for only one are presented As can be seen, the measured noise levels are somewhat larger than expected, but within an order of magnitude of specification. The effects of filtering in reducing the wideband noise can be seen by comparing the noise levels before and after the bandpass filter. Since the noise added by the amplifier is small compared to the total output noise, the noise output is basically the noise level at the filter output, multiplied by the amplifier gain. This yields

$$
\sigma^{2} \text { frontend }=\left(0.54 \times 10^{-3} G\right)^{2} \text { watts }
$$

where $\mathrm{G}$ is the voltage amplification gain and the $1 \mathrm{ohm}$ convention is used.

Table 3.1: Noise Levels for Analog Front-End Circuitry

\begin{tabular}{|l|l|l|l|}
\hline Component & $\begin{array}{l}\text { Expected Noise for } \\
\text { Component } \\
(\mu \text { Vrms })\end{array}$ & $\begin{array}{l}\text { Cumulative Noise } \\
\text { Level } \\
(\mu \text { Vrms })\end{array}$ & $\begin{array}{l}\text { Measured Noise } \\
\text { Level } \\
(\mu \text { Vrms })\end{array}$ \\
\hline $\begin{array}{l}\text { preamplifier } \\
100 \text { x gain }\end{array}$ & 0.76 & 0.76 & - \\
bandpass filter & 64 & 76 & 680 \\
amplifier & 9 & 140 & 540 \\
$10 x$ gain & - & 149 & - \\
$\begin{array}{l}\text { equivalent input } \\
\text { noise }\end{array}$ & - & 1490 & 5370 \\
\hline
\end{tabular}




\subsubsection{Expected Ambient Noise}

There are many types of ambient noise in the ocean, such as shipping noise, windwater interaction noise, and biological noise. In the Boston Harbor shipping noise will of course be the largest contributor. Luckily, this noise is concentrated at frequencies far below those at which the USBL system operates, and so it only remains to design a system which can filter the out-of-band noise adequately to avoid amplifier saturation at the expected gain levels. Urick [1983] reports accumulated measurements of ambient noise for a shallow-water, heavy traffic environment, which are seen in Fig 3.19. This noise is filtered in the prototype USBL system by a single-pole highpass with a $5 \mathrm{kHz}$ cutoff designed to prevent saturation in the preamplifier stage, combined with a 8-pole bandpass filter which provides $48 \mathrm{~dB} /$ octave rejection outside its passband region of $14-34 \mathrm{kHz}$. A simple calculation assuming a maximum gain of $100 \mathrm{~dB}$ and accounting for the sensitivity of the hydrophones used shows that the maximum output noise level from out-of-band noise should be approximately $3.2 \mu \mathrm{V}_{\mathrm{rms}}$ for the noise levels reported by Urick. Thus the filtering provided is clearly more than adequate, and should serve well in noisy environments.

The ambient noise in-band is much more important from a signal processing point of view. The expected ambient noise level for the shallow-water heavy traffic environment at $26 \mathrm{kHz}$ is approximately $55 \mathrm{~dB}$ re $1 \mu \mathrm{Pa}$ for $1 \mathrm{~Hz}$, or $98 \mathrm{~dB}$ re $1 \mathrm{mPa}$ for a $20 \mathrm{kHz}$ bandwidth. Factoring in the $-201 \mathrm{~dB}$ re $1 \mu \mathrm{Pa} / \mathrm{V}$ sensitivity of the Benthos AQ-4 hydrophones yields an expected noise level after the preamplifier stage of $5 \times 10^{-11} \mathrm{~V}_{\mathrm{rms}}$. The expected noise power is then given by

$$
\sigma^{2} \text { ambient }=\left(5 \times 10^{-11} \mathrm{G}\right)^{2} \text { watts }
$$

where $\mathrm{G}$ is the amplifier voltage gain and the $1 \mathrm{ohm}$ convention is again used to calculate power. 


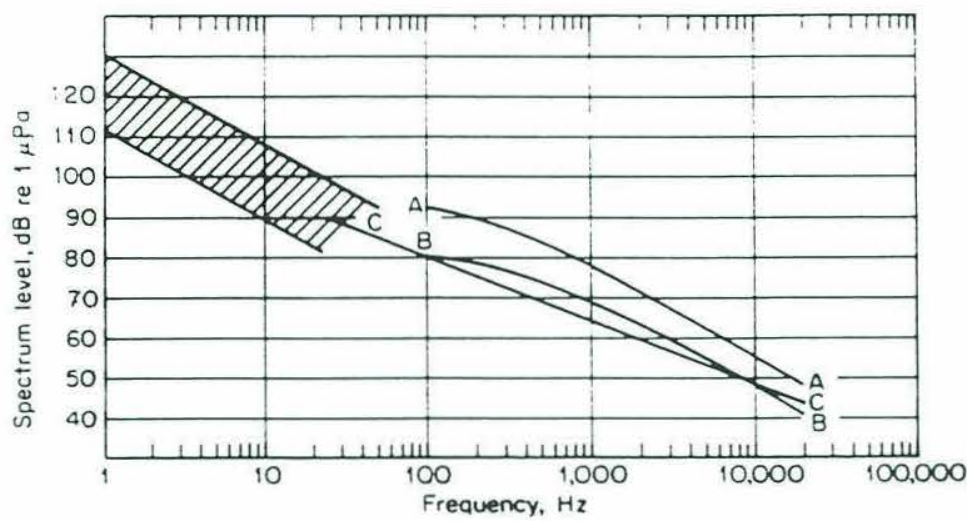

Figure 3.19: Ambient Noise Power Spectra for Shallow Water [after Urick, 1983] Line AA represents data for heavy-traffic location; used for Boston Harbor calculations

\subsubsection{A/D Quantization Noise}

In the prototype USBL system 14-bit Analog Devices AD679 A/D converters are used for sampling the received signal. A simple analysis of the quantization noise introduced can be made based on a few simple assumptions.

The quantization error is defined as

$$
\mathrm{e}[\mathrm{n}]=\mathrm{x}[\mathrm{n}]-\mathrm{x}[\mathrm{n}]
$$

where $x[n]$ is the output of the $A / D$ converter and $x[n]$ is the true value of the signal. The AD679 is a linear converter, so the quantization error is bounded by

$$
-\Delta / 2<\mathrm{e}[\mathrm{n}]<\Delta / 2
$$

where $\Delta$ is the voltage step between quantization levels. If $\Delta$ is small compared to the signal amplitude then e[n] is accurately modeled as a random variable uniformly distributed over $[-\Delta / 2, \Delta / 2]$. At this point it is convenient to assume [Oppenheim, 1989] that $\mathrm{e}[\mathrm{n}]$ is:

1) a sample sequence of a stationary random process

2) uncorrelated with the sequence $x[n]$ 
3) uncorrelated from sample to sample (i.e. white).

It is clear that if the input signal is a pure-tone sinewave with no noise, assumption 2 will be violated. This analysis will therefore be less accurate in cases of extremely high signalto-noise ratio. In practice, however, the assumptions are realistic if the signal is somewhat complicated and if the signal amplitude will cross many quantization levels from sample to sample [Oppenheim, 1989]. Since the step level $\Delta=0.6 \mathrm{mV}$ for the AD679 A/D converters, and even at the lowest gain levels the noise from the front-end electronics will be an order of magnitude larger than this, the assumptions should be reasonably accurate for the prototype system.

The noise power of the quantization error is now given by the variance of e[n], which is found to be [Oppenheim, 1989]

$$
\sigma_{\text {quantization }}^{2}=\frac{\Delta^{2}}{12}=\frac{\left(2^{-\mathrm{B}} \mathrm{X}_{\max }\right)^{2}}{12}
$$

where $\mathrm{B}$ is the number of conversion bits and $\mathrm{X}_{\max }$ represents the full-scale amplitude of the converter. For the AD679 it follows that

$$
\sigma^{2} \text { quantization }=3.1 \times 10^{-8} \text { watts. }
$$

\subsection{Predictions of Bearing Estimation Performance vs. Range}

The noise level predictions of the preceding sections can be combined with the USBL bearing estimation performance model to give some idea of errors at different source-receiver ranges. In section 3.6 it was seen that for the prototype USBL system designed for this thesis, the largest noise contribution came from the front-end noise. This immediately suggests that further development of this system could benefit from construction of lower-noise front-end circuitry. However, predictions can be made for the performance of the existing system.

The intensity of sound transmitted by a pinger decreases rapidly with range as a result of geometric spreading and absorption. The sonar equation can be used to predict the signal-to-noise ratio at a range $\mathrm{R}$ [Dyer p.25-26]: 


$$
\mathrm{SNR}=\left(\mathrm{L}_{\mathrm{S}}-10 \log _{10}(\mathrm{R})-\alpha \mathrm{R}\right)-\mathrm{N}, \mathrm{dB}
$$

where $\mathrm{L}_{\mathrm{S}}$ is the source level in $\mathrm{dB}$ re $1 \mu \mathrm{Pa}, \alpha$ is the absorption coefficient for the broadcast frequency, and $\mathrm{N}$ is the noise power in $\mathrm{dB}$. For this calculation cylindrical spreading is assumed instead of geometric spreading. This is done to allow comparison with the 125 meter range shallow water test results in Chapter 5, for which the propagation range was approximately ten times the water depth. The predicted SNR for the prototype system is seen in Fig. 3.20 below. In Figure 3.21 the predicted bearing errors as a function of range are shown. In a shallow water environment system performance is limited by multipath interference, and sound propagation is much more complex than suggested by the sonar equation. Still, these plots provide an starting point for predicting navigation accuracies.

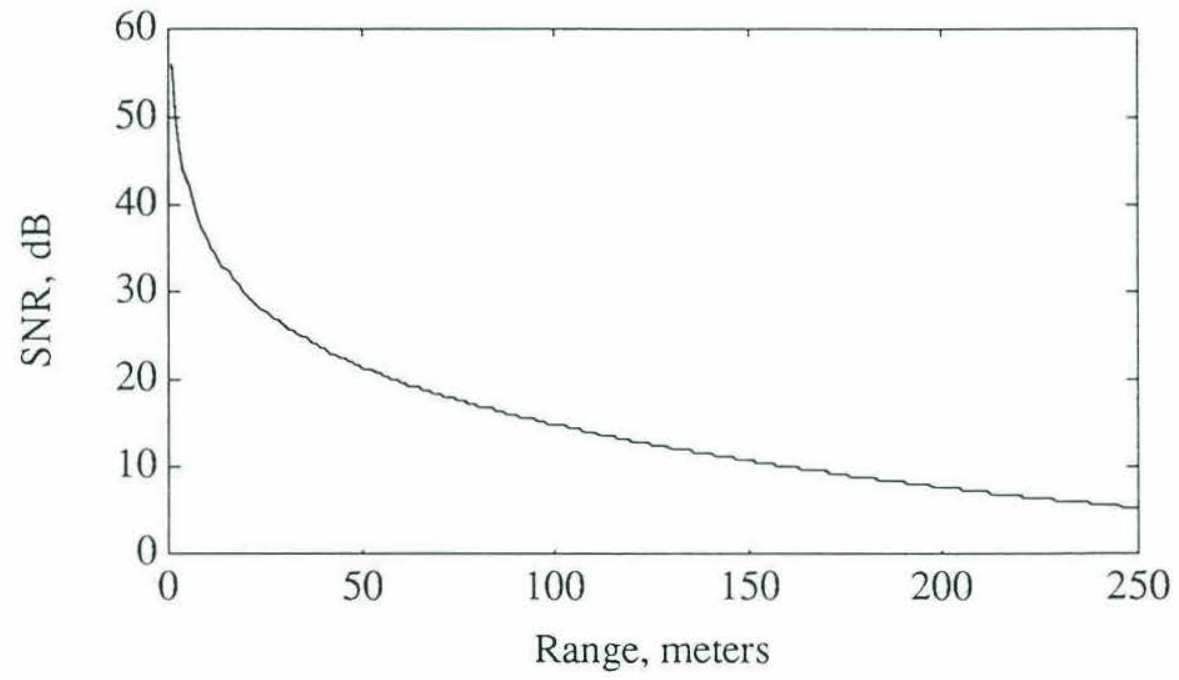

Figure 3.20: Signal-to-Noise Ratio Vs. Range 


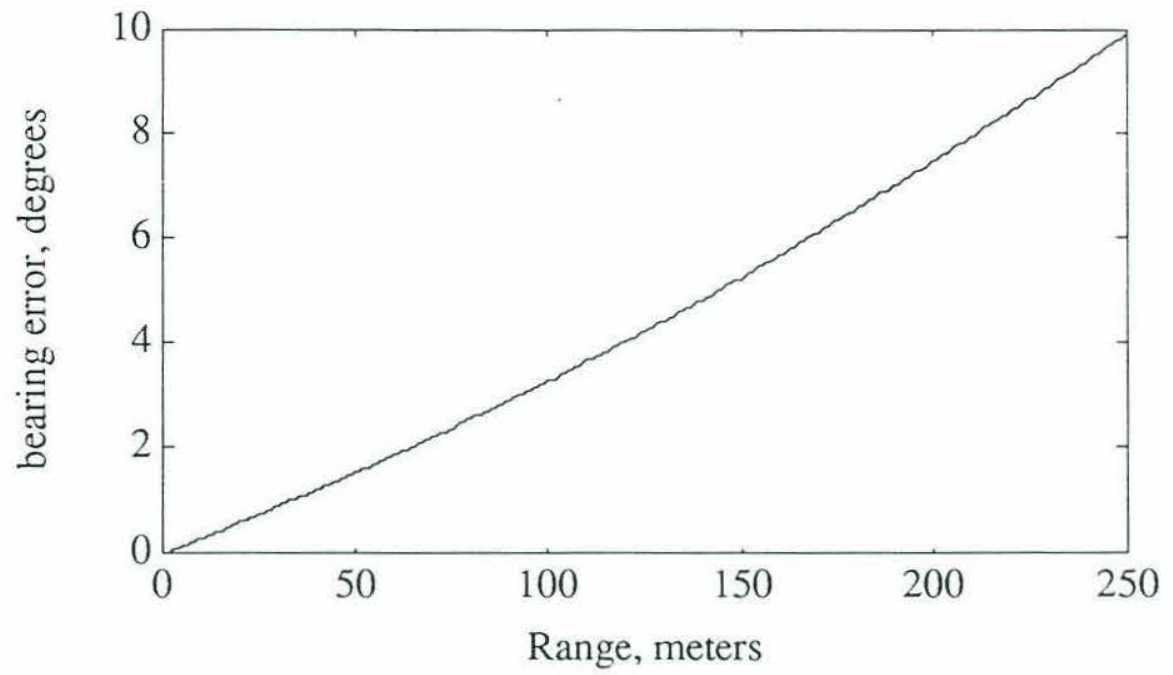

Figure 3.21: Bearing Error Vs Range for Small Bearing Angles 


\section{Prototype USBL Configuration}

\subsection{Introduction}

During May and June 1992, a series of in-water tests was carried out to determine the performance of the prototype single-frequency USBL system. A description of the test apparatus and of the acoustic environment is given below, and solutions to problems encountered in obtaining data are discussed.

\subsection{Hardware Arrangement}

A schematic view of the pinger and hydrophone deployment is shown in the side view, Fig. 4.22. The pinger used was a Datasonics Acoustic Location Pinger ALP-363, which was tuned to emit a $26 \mathrm{kHz}$ pulse of $9.6 \mathrm{msec}$. duration every 5 seconds. The pinger's power is 20 Watts, or $180 \mathrm{~dB}$ re $1 \mu \mathrm{Pa}$. For early short range tests the pinger was suspended in the water by a pair of ropes. This proved unsatisfactory, as the motion of the pinger due to wave action gave rise to noticeable bearing variations over the short travel distance. For later tests, the pinger was deployed from a 20' length of 2" diameter PVC pipe. This damped out wave action and allowed more accurate results.

The hydrophone pair used was also attached to a length of PVC pipe. This pipe was held fast on the barge deck by a PVC crosspiece, which had a protractor mounted on it to measure rotations. The bearing from the array to the pinger was varied by rotating the pipe. The bottom of the pipe terminated in a small T-piece, to which the hydrophone assembly was attached. The hydrophone assembly will be discussed more below.

Shielded twisted-pair cables were soldered to the hydrophone leads, which were insulated with marine silicon rubber sealant. The cables were run up the PVC pipe to the front end electronics.

The signals received by the hydrophones were transmitted up the shielded twistedpair cables and then amplified, filtered, and digitized. The electronics setup is 


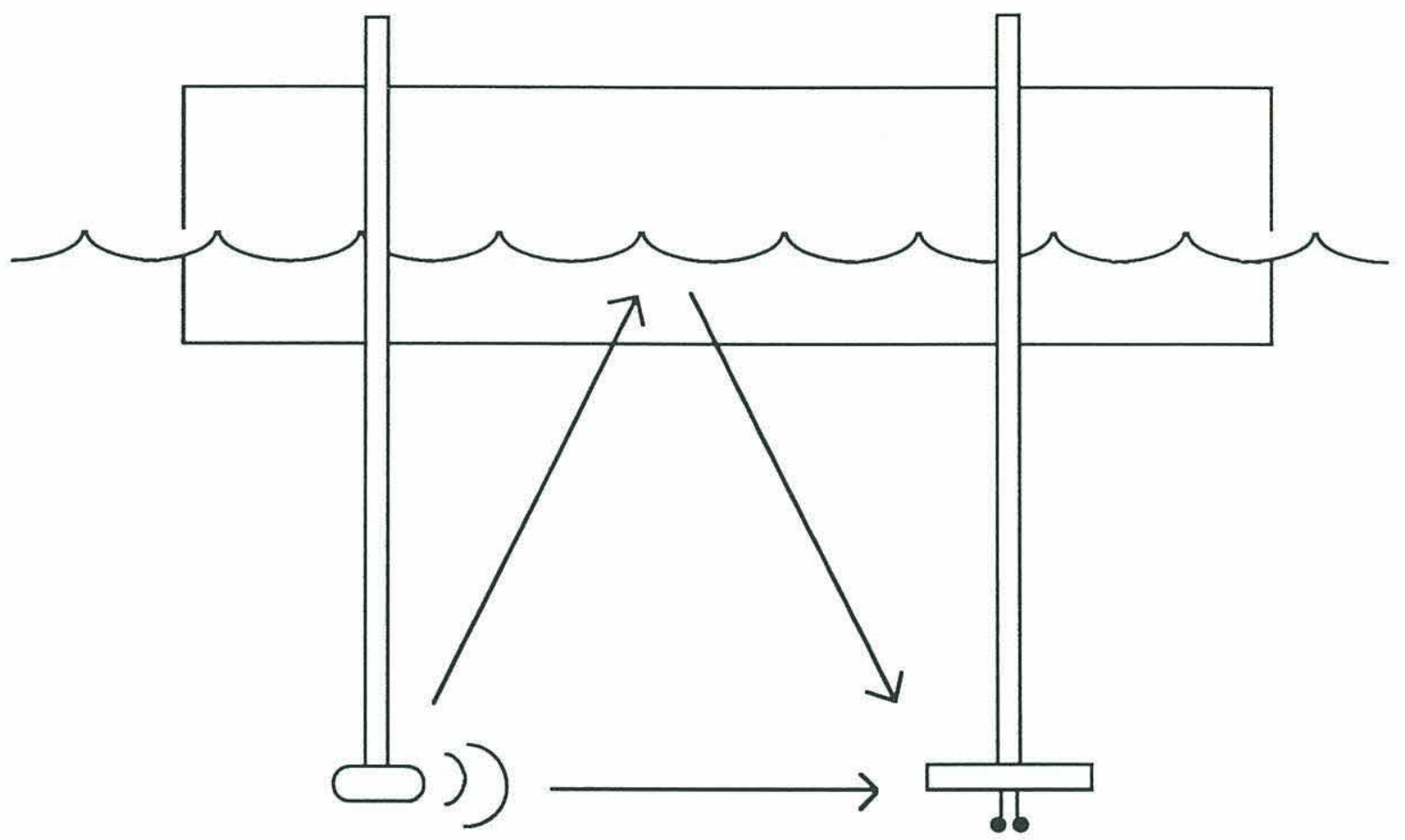

Figure 4.22: Pinger and Hydrophone Deployment 


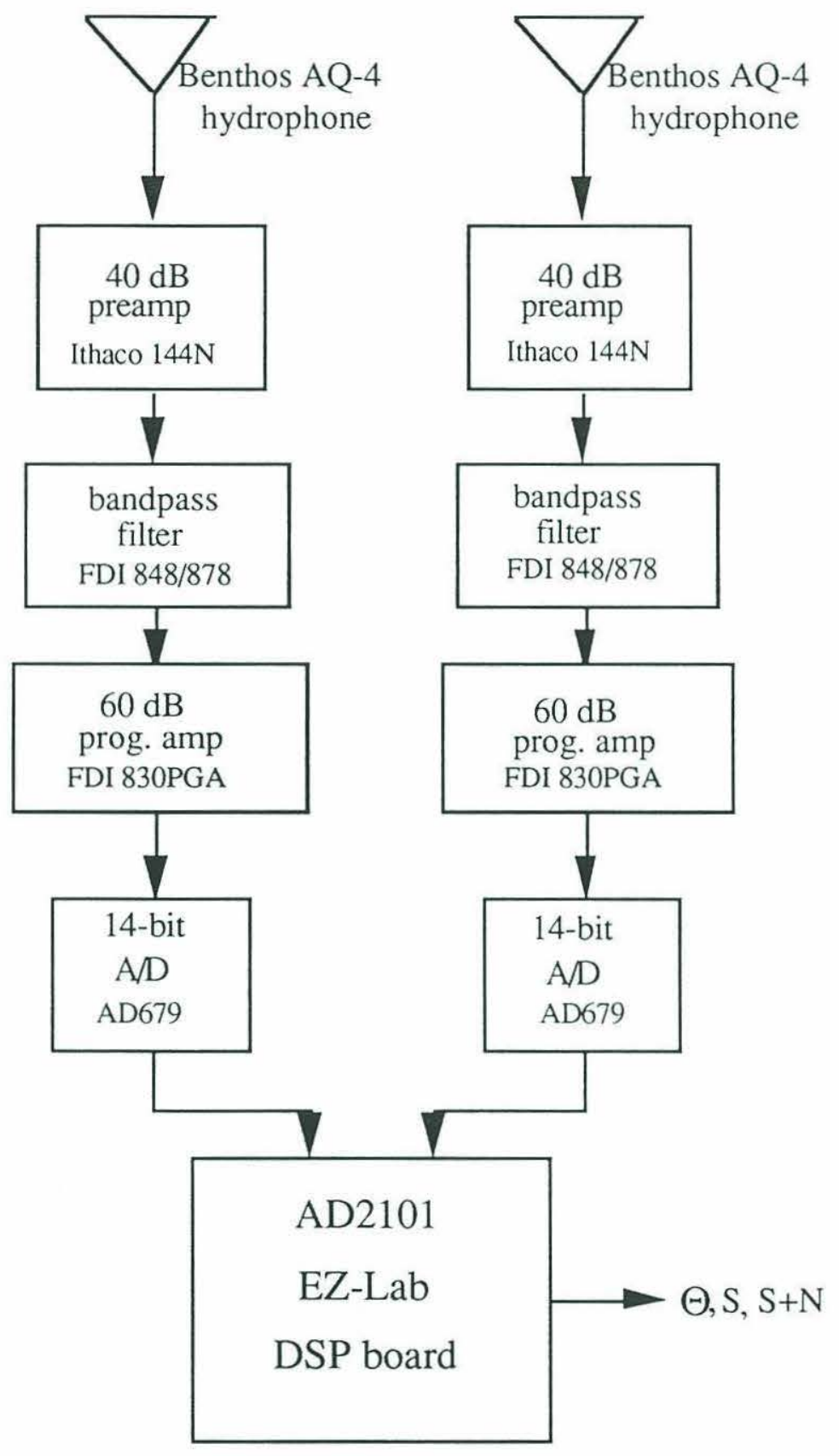

Figure 4.23: Electronics for Prototype System 
shown in Fig. 4.23, and details of the design are available in Appendix A. The system provides a maximum of $100 \mathrm{~dB}$ of gain, and the bandpass filters provide attenuation of 48 dB/octave.

After the test apparatus was assembled, the source-receiver geometry was measured and the pinger activated. The output of the front end was observed on a field oscilloscope, and amplifier gains were adjusted as required. The time delays between different arrivals as seen with the oscilloscope were recorded, as well as their amplitudes. After it was verified that the front-end was working as desired, the signals were connected to the digital circuitry

The digital circuitry consisted of a data acquisition board and the DSP system. The data acquisition board was built to interface with the Analog Devices AD2101 EZ-Lab standalone DSP board, and included two 14-bit AD679 A/D converters. The DSP board was run in emulator mode using a 386 laptop computer as a terminal. Software was compiled on the computer and down-loaded to the DSP. The USBL program then read in data values from the data acquisition board and processed the data. The output of this program consisted of packets, each of which held data obtained by integrating over 4 cycles of the $26 \mathrm{kHz}$ signal. Each time the signal power crossed the detection threshold a data packet consisting of a time stamp, the wideband power, the signal power, and the calculated phase difference was recorded. This data was up-loaded to the 386 using Procomm and logged on the hard drive for later analysis.

\subsection{Hydrophone Mounting}

Two Benthos AQ-4 hydrophones, of dimensions $1.3 \times 3.3 \mathrm{~cm}$., were mounted in holes drilled through a small PVC plate. This plate was gripped by a small metal holder which could be tightened The hydrophones were separated by $2.88 \mathrm{~cm}$., or half a wavelength for a $26 \mathrm{kHz}$ wave traveling at $1500 \mathrm{~m}$./s. The mounting is shown below. 


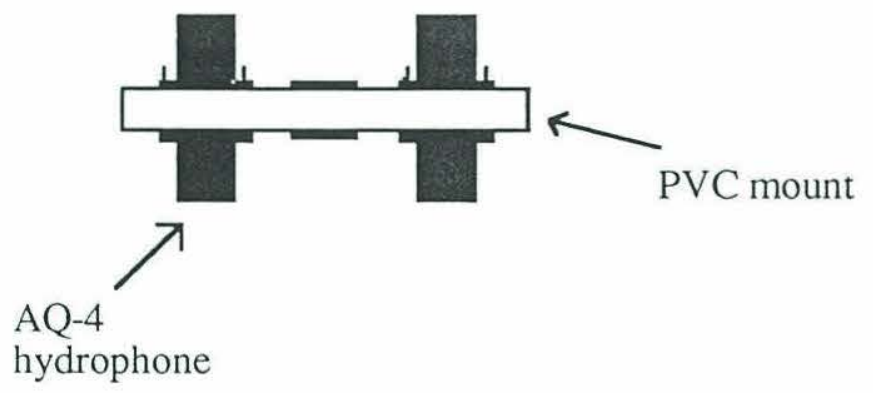

Figure 4.24: Hydrophone Mount

a) front view

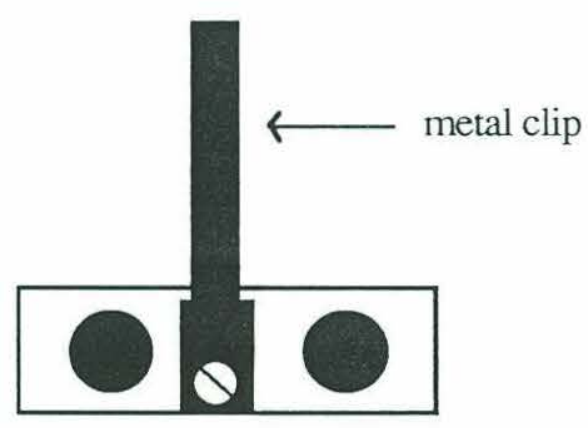

b) top view

One major concern during the design stage was that the size of the hydrophones and mounting was sufficiently large compared to the $5.77 \mathrm{~cm}$. wavelength to disturb the sound field and interfere with the phase measurement. This interference in fact occurred during the short-range tests conducted early in the testing program. The hydrophone mounting used was asymmetrical, and seems to have interfered with sound waves incident from one side of the hydrophone mount. The mount was reduced in size for later tests, and the problem disappeared. As a diagnostic, the received signals on the two channels were observed on an oscilloscope to verify that the phase difference changed as expected as the hydrophone mount was rotated.

The hydrophones used were found to somewhat directive at $26 \mathrm{kHz}$, which is to be expected given their size. For the longer-range tests done for this thesis this directivity has some advantages, as it will tend to attenuate noise generated at the surface. However, it could be a drawback in situations in which it is desirable to receive signals from large 
out-of-plane angles. One such situation could arise if a submersible were to travel over an acoustic beacon moored close to the bottom.

Problems were experienced during testing with the method used for attaching the cable leads to the hydrophones. The cables were simply soldered to the hydrophone contacts, and the connection was insulated with silicone rubber sealant. Over time, the salt water ate away at the silicon rubber, eventually exposing the leads and causing the hydrophones to short through the water. This annoying problem can be avoided in the future by embedding the hydrophones and connectors in a block of epoxy.

\subsection{Description of Acoustic Environment}

All tests were carried out at the Sea Grant shack facility in the Charlestown Navy Yard in Boston. The Sea Grant shack is located on the end of the pier at which the U.S.S. Constitution and U.S.S. Cassin Young are moored. A barge at the end of the pier was used as the test site for the experiments. The tests were carried out in very shallow water, meaning the returns were strongly affected by surface and bottom reflections. The deck of the barge floats approximately $1.72 \mathrm{~m}$. above the water, which at this location varies in depth between roughly $10 \mathrm{~m}$. at low tide and $13 \mathrm{~m}$. at high tide. The harbor bottom in this region is fairly muddy and can be expected to be strongly absorbent of sound. An effort was made during testing to locate both the source and the receiver near the midpoint of the water column, in order to introduce as long a delay as possible between direct and surfacebounce acoustic arrivals.

The physical setup of the test site can be seen in a top view in Fig. 4.25. Short, medium, and long-range tests were carried out, and the approximate source and receiver locations are shown.

Short-range tests were designed to prove that the system works when direct and reflected ray paths can be easily identified and the SNR is high. The source-receiver separations for these tests were typically on the order of 3-4 meters. Well-defined 


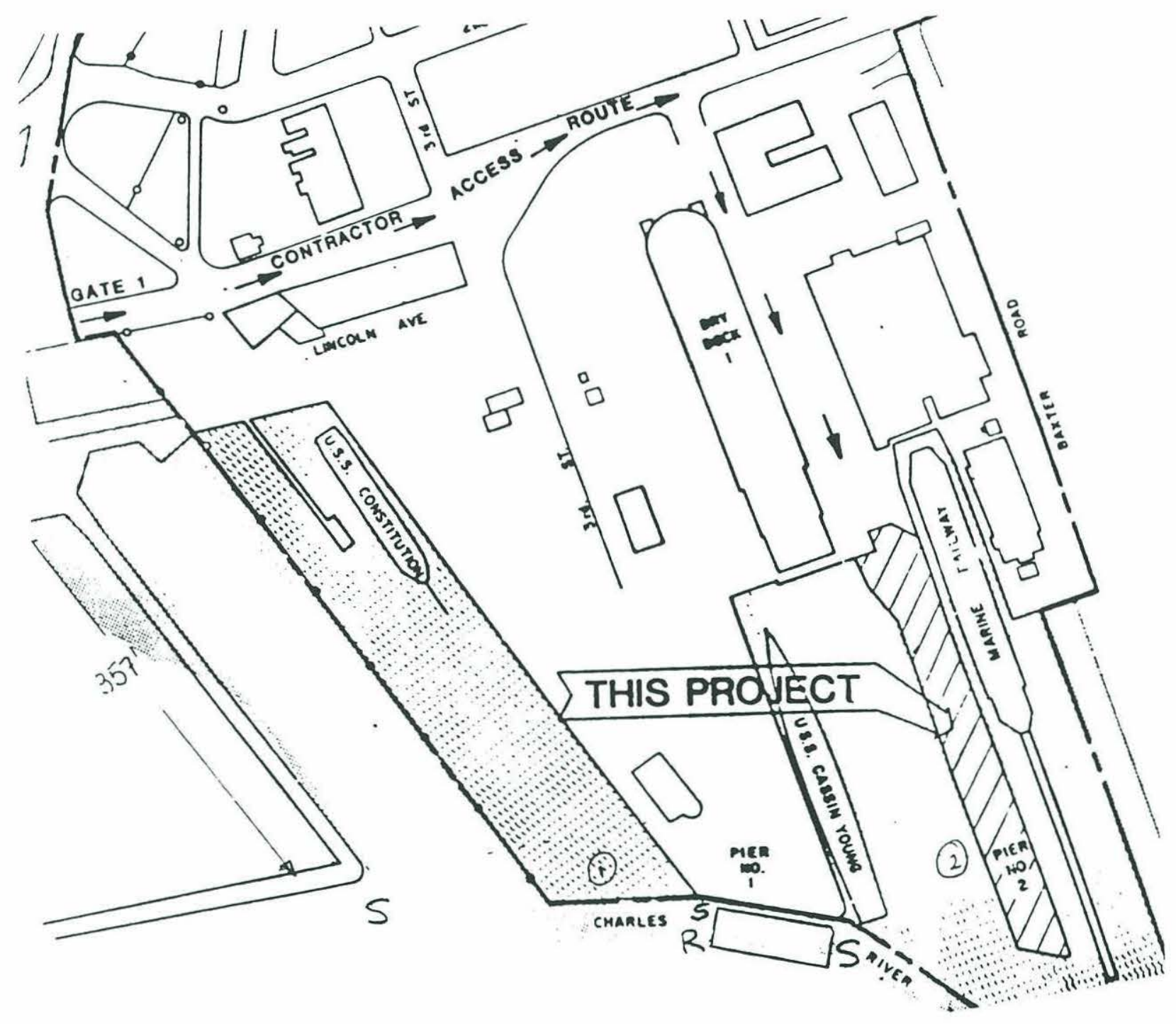

Figure 4.25: Experimental Setup at Charlestown Navy Yard Source locations marked by "S", Receiver by "R" (at end of Pier 1) Map Courtesy of U.S. Park Service 
arrivals could be seen from the direct path and surface-bounce path, and the impact of the bottom-bounce arrival can be seen in the bearing estimates. These experiments provided a test of the system's accuracy in bearing resolution, and gave information about phase stability within the non-overlapped sections of the pulse.

Medium-range tests were carried out to study system performance under more typical operational conditions, when multipath effects could be easily calculated. Some of these tests were carried out along the length of the barge, a distance of approximately 30 meters. There were several drawbacks to operating the USBL system along the length of the barge. The first is that the draft and shape of the barge hull are unknown, although markings on the barge seem to indicate the draft is close to 1 meter. Experimenters from Draper Laboratory have reported receiving echos from some object underneath the barge, which seems to change its position [John Irza, personal communication, July 92]. The Draper researchers have speculated that the object may be Jimmy Hoffa's body. Another problem is that there is a relatively small time delay between the direct and surface reflected paths. Observations of the pulses on an oscilloscope showed that approximately 4-5 cycles of the direct path transmission were received before other paths began interfering. This time delay corresponds reasonably well to the 6 cycle delay expected between direct and surface-reflected paths if a barge draft of $1 \mathrm{~m}$. is assumed (see Table 4.1). Thus only the bearing estimate from the first point or so of each received pulse represents an estimate made from uncontaminated data. Unfortunately this section of the pulse will also contain some transients from pinger ringing, which as seen in the short range data may degrade the data somewhat. Still, useful results can be obtained.

Tests were also conducted with the pinger deployed from a dock in the Charlestown Marina, approximately 125 meters distant. These experiments gave results in a situation in which multipath was significant and could be estimated from the sound speed profile. 


\subsection{Sound Speed Measurement and Ray Trace Results}

The speed of sound was measured during each set of experiments. An accurate knowledge of the sound speed profile is important for two reasons. It is important to know the sound speed at the receiver depth, as this is an input to the bearing calculation. For the long-range experiments it is useful know the sound speed profile so that a ray-trace model can be used to predict arrival times of rays which reach the receiver. While refraction was generally not an important effect for these tests, multipath interference was quite noticeable.

The sound speed profile was determined using Sea Grant's Hydrolab H20 Water Quality Data sampler. The H20 was slowly lowered down through the water column and data values profiling depth, salinity, and temperature were logged. Depth was converted to pressure using a value for salt water density of $1032 \mathrm{~kg} / \mathrm{m}^{3}$. These values were used to calculate a sound speed profile. The NRL II formula [Del Grasso, JASA 1974] was used to calculate sound speed, although all terms of magnitude $10^{-3} \mathrm{~m} / \mathrm{s}$ or less were neglected. The profile was then averaged to produce values at $1 \mathrm{~m}$. increments, which were used as input to a ray-tracing model developed by Max Deffenbaugh.

Typical plots for the temperature and salinity in the water column are shown in Fig. 4.26. These data were logged on July 6, the day on which the long-range tests described in the next chapter were conducted. The plots show the existence of a warmer, less saline layer near the water surface, and are typical for water column data taken on warm days. The NRL II formula was used to generate a sound speed velocity profile from this data, seen in Fig. 4.27a. The sound speed profile varied somewhat from day to day. Figure $4.27 \mathrm{~b}$ from June 19 shows another sound speed profile. The profiles are in general downward refracting, meaning that sound propagation over longer ranges will be limited by bottom absorption.

Calculations of the expected multipath arrivals for the three test geometries are shown in Table 4.1. For the short and medium range tests the ray paths were calculated 


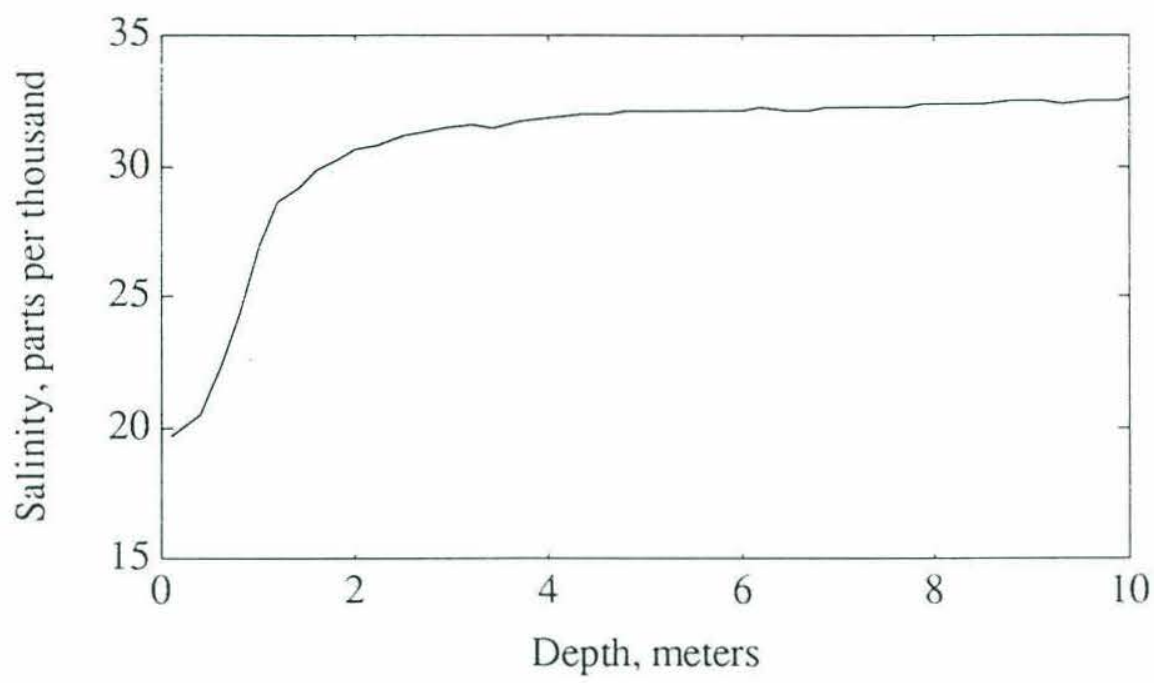

Figure 4.26: Temperature and Salinity Profiles for July 6 a) Salinity Profile

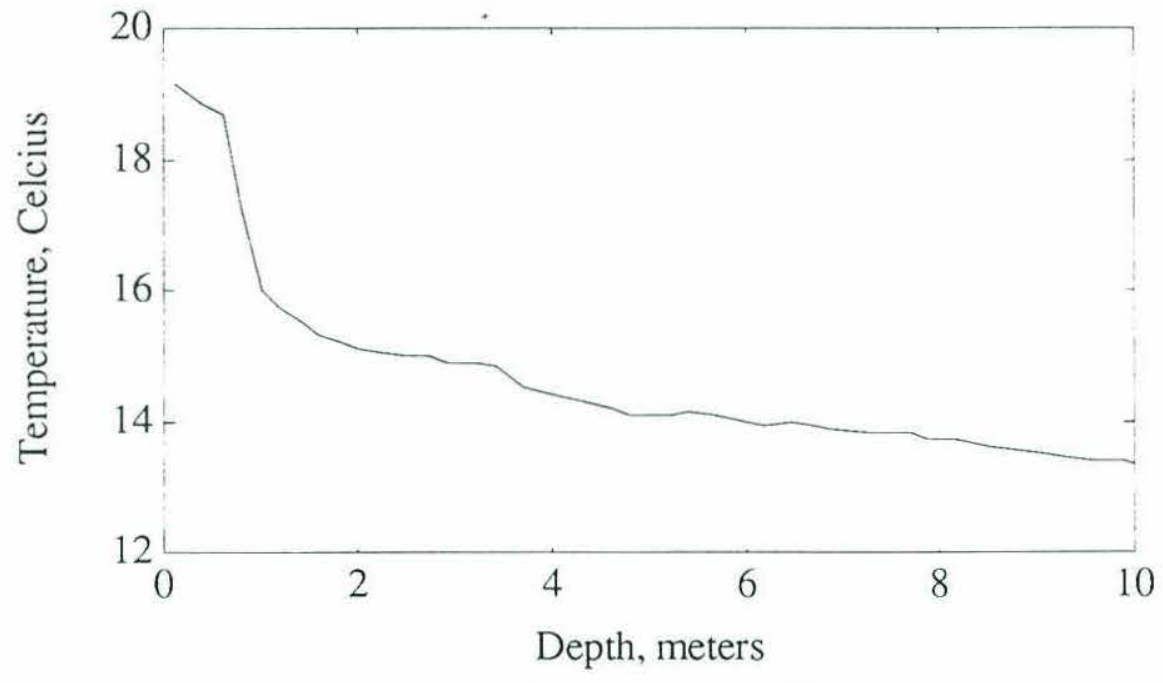

b) Temperature Profile 


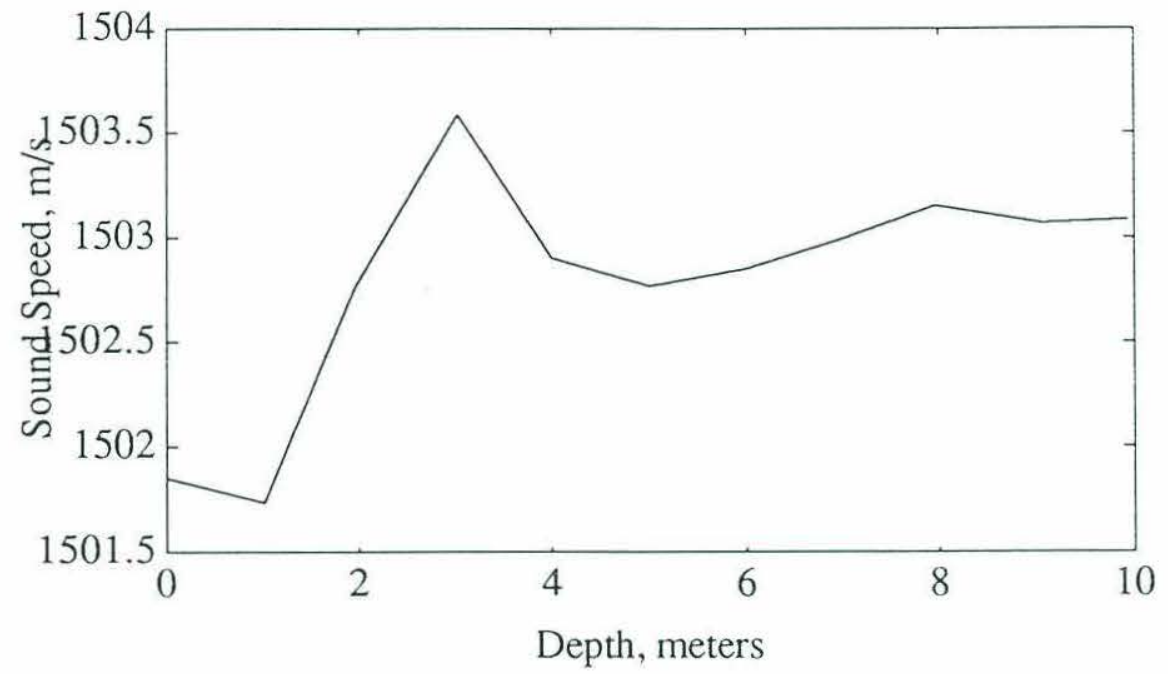

Figure 4: Typical Sound Speed Profiles

a) July 6 data

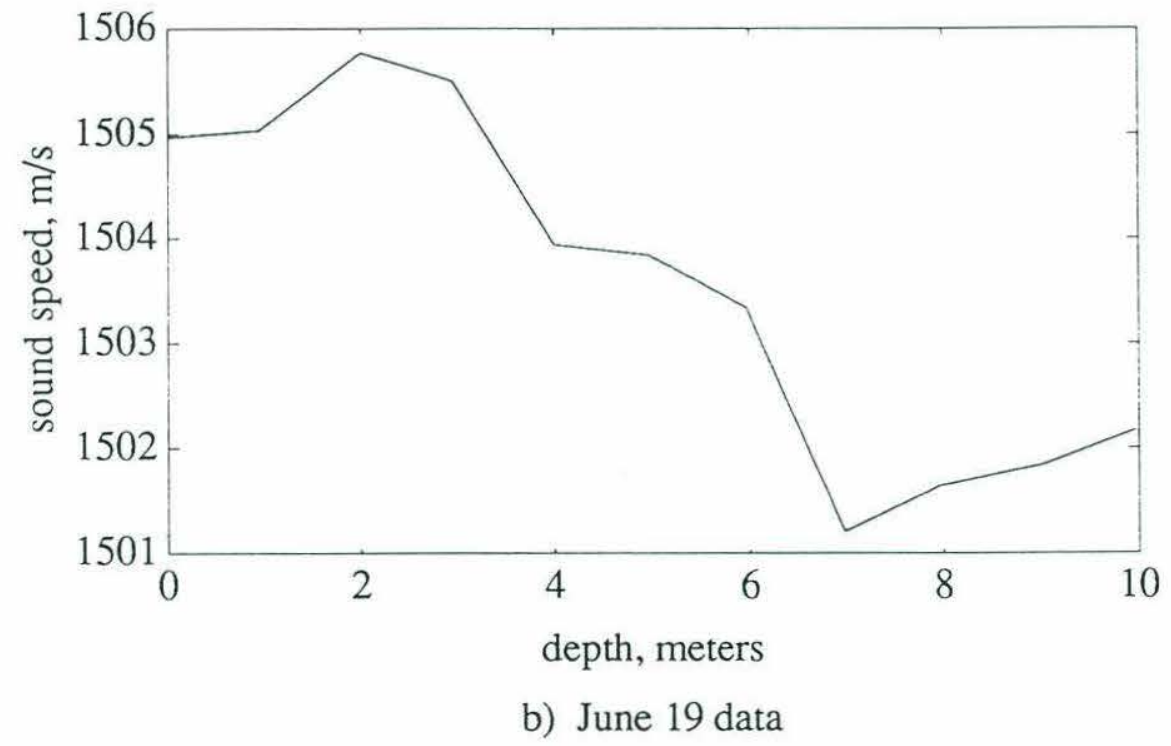


using simple geometry, since the ranges were short enough so refraction was not significant. The long-range predictions were made using a ray-trace program using the July 6 sound speed profile. The ray paths traced are shown in Figure 5 below. The signal strengths shown are rough estimates, based on a bottom reflection loss of $16 \mathrm{~dB}$ [Urick, 1983]. The effects of the multipath arrivals are seen clearly in the data presented in the next chapter.

Table 4.1: Expected Ray Paths for Test Geometries

\begin{tabular}{|c|c|c|c|c|}
\hline Range & $\begin{array}{c}\text { Time Delay after } \\
\text { 1st arrival }\end{array}$ & $\begin{array}{l}\text { Vertical Arrival } \\
\text { Angle } \\
\text { (degrees) }\end{array}$ & Ray Description & $\begin{array}{c}\text { Estimated } \\
\text { Strength } \\
\text { Relative to } \\
\text { Direct Path }(\mathrm{dB})\end{array}$ \\
\hline 3.5 & 0 & 0 & \multirow{3}{*}{$\begin{array}{c}\text { direct path } \\
\text { surface bounce } \\
\text { bottom bounce }\end{array}$} & 0 \\
\hline 3.5 & 2.6 & 58 & & 0 \\
\hline 3.5 & 6.2 & -71 & & -16 \\
\hline 35 & 0 & 0 & \multirow{4}{*}{$\begin{array}{l}\text { direct path } \\
\text { off barge } \\
\text { bottom } \\
\text { bottom bounce } \\
\text { wall bounce }\end{array}$} & 0 \\
\hline 35 & 0.2 & 8 & & -3 \\
\hline 35 & 1.2 & -18 & & -16 \\
\hline 35 & 2.9 & 27 (horizontal) & & $? ?$ \\
\hline 125 & 0 & 0.5 & \multirow{2}{*}{$\begin{array}{l}\text { direct path } \\
1 \text { surface } \\
\text { bounce }\end{array}$} & 0 \\
\hline 125 & 0.2 & 3.4 & & 0 \\
\hline 125 & 0.4 & -5.5 & $\begin{array}{l}1 \text { bottom } \\
\text { bounce }\end{array}$ & -16 \\
\hline 125 & 1.2 & -9.5 & $\begin{array}{l}\text { surface, then } \\
\text { bottom bounce }\end{array}$ & -16 \\
\hline 125 & 1.0 & 8.5 & $\begin{array}{l}\text { bottom, then } \\
\text { surface bounce }\end{array}$ & -16 \\
\hline 125 & 2.1 & 12.5 & $\begin{array}{l}\text { top, bottom, } \\
\text { then top } \\
\text { bounces }\end{array}$ & -16 \\
\hline
\end{tabular}




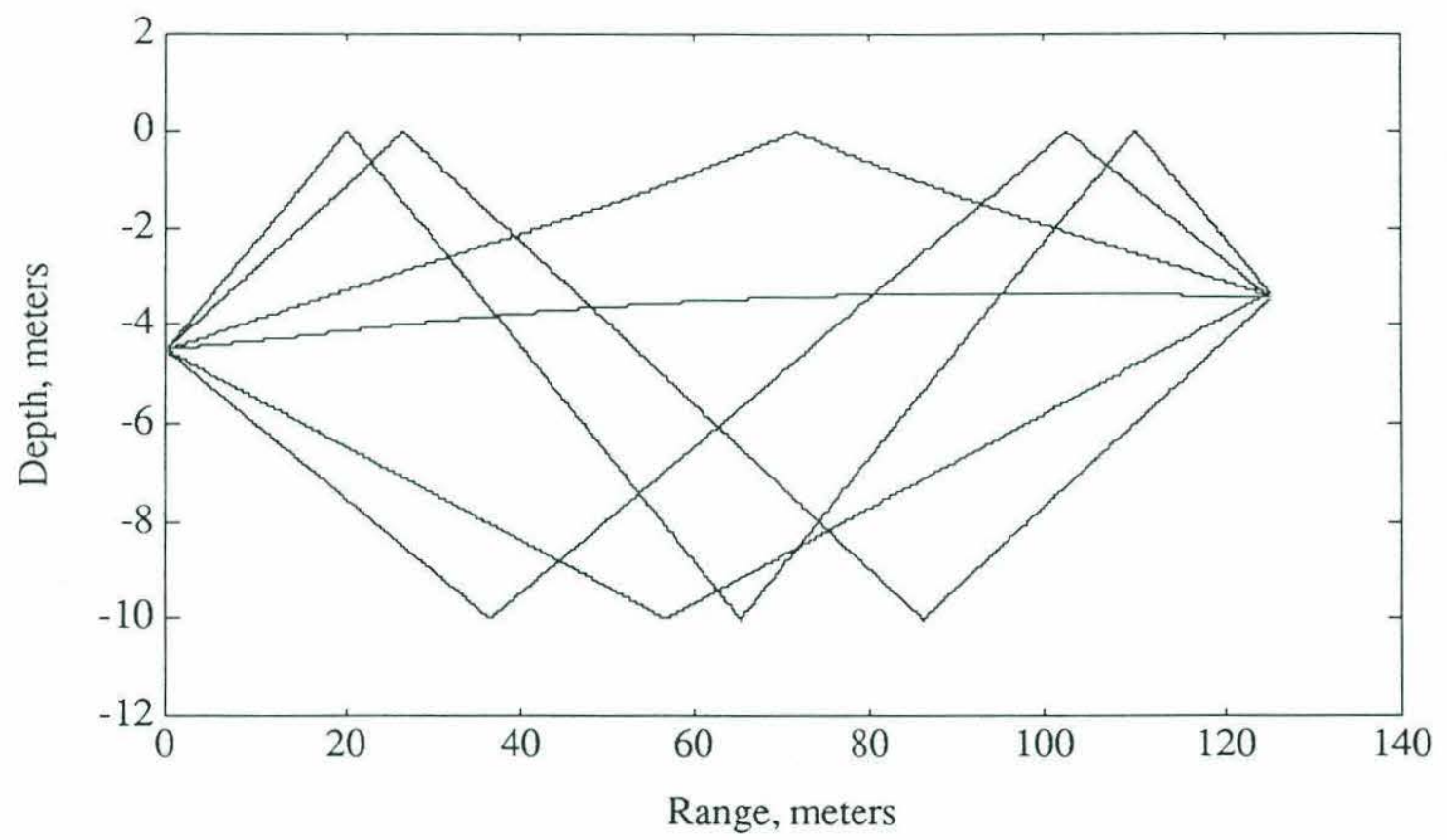

Figure 4.28: Ray-Trace Results for July 6 


\section{Test Results}

\section{$\underline{5.0 \text { Introduction }}$}

Test results for short-, medium-, and long-range operation of the prototype USBL system are presented and discussed here. The effects of multipath interference are seen clearly in the data, and proved to be the largest impediment to reliable bearing estimation. The effects of the multipath structure are explored in the experiments, and the significance of an observed correlation between fluctuations in signal power and bearing estimation due to multipath interference is examined.

\subsection{Overview of Experimental Program}

To briefly summarize the last chapter, a series of tests of the prototype USBL system were carried out in May, June and July 1992. Three main sets of experiments were conducted, with source-receiver separations of 3-4 meters, 35 meters, and 125 meters respectively. A $26 \mathrm{kHz}$ commercial pinger was used as the sound source. After being activated it transmitted a $9.5 \mathrm{msec}$ duration pulse at approximately 5 second intervals. The signals were received by two hydrophones, separated by a distance of half a wavelength. The signals were processed by the DSP setup with an integration time of 4 cycles. For each integration period that exceeded the detection threshold a data packet was recorded which contained timing and power information as well as the phase difference estimate. These data were post-processed in the lab to produce bearing estimates, using the measured sound velocity on the test date as an input.

Short-range experiments were carried out in May 1992. The first few experiments were spent refining the experimental setup, but experiments on May 15 and May 21 yielded interesting data. These tests illustrated that the USBL system was capable of generating correct bearing estimates. These tests were also the only experiments conducted in which an appreciable amount of time was present between the arrival of the direct and surfacereflected paths. Thus they served to give information about the stability of the phase 
estimate within a pulse. Also, the May 15 data is used to illustrate the correction made for ray arrivals which were not in the horizontal plane of the array.

Medium-range tests were carried out along the length of the Navy Yard barge on June 26 , July 6 , and July 8 . These tests were designed to show that the system could operate at more realistic ranges. An effort was made to gauge the stability of the multipath structure.

Long-range tests were conducted on July 6. More data should be collected at this 125 meter range in order to better quantify the system's performance, but these initial results indicate that the bearing measurements over this range are fairly stable, and that ray paths reflected off the surface seem to retain enough coherence to give reasonable bearing estimates.

\subsection{Performance Issues}

Although the test geometries were quite different, a number of performance issues are common to all of them. For all the test geometries, fluctuations in the estimated bearing during periods of multipath interference were observed. In Appendix $\mathrm{C}$ the expected effects on the phase difference estimate from interference between 2 interfering arrivals are calculated. An attempt was made to apply these calculations to data from short and longrange tests to gain a better understanding of the interference process. Also, an apparent correlation between fluctuation in signal strength and in bearing estimates during periods of overlapping interfering multipaths was investigated. The significance of this correlation is examined at the end of the chapter.

Another performance issue common to all three tests is the effects of noise on estimates of phase difference and bearing. Table 5.1 below summarizes the results for the three geometries, and compares the measurements to the predictions of the noise model introduced in Chapter 3. For the short-range tests, unfortunately, repeated measurements at the same bearing were not made. Thus noise performance is reported in terms of 
deviation in estimated phase difference rather than bearing. This removes the dependence of the error on bearing seen in section 3.1 and allows all the available data to be used. For long and medium ranges only the results from the first 4 cycles were used, as multipath interference was present during the remainder of the pulse.

Table 5.1 shows that the agreement between measured and predicted errors seems to increase as the SNR decreases. This indicates that at high SNR the errors mostly stem from sources other than noise, such as detuning errors. As SNR increases the errors resulting from noise begin to dominate other error sources and the agreement between model and experiment improves.

Table 5.1: Measured and Predicted Estimation Errors

\begin{tabular}{|c|c|c|c|c|c|}
\hline $\begin{array}{l}\text { Range } \\
\text { (meters) }\end{array}$ & $\begin{array}{l}\text { Test } \\
\text { Statistic }\end{array}$ & $\begin{array}{l}\text { Standard } \\
\text { Deviation of } \\
\text { Statistic }\end{array}$ & $\begin{array}{l}\text { Number of } \\
\text { Samples } \\
\text { used }\end{array}$ & $\begin{array}{l}\text { Measured } \\
\text { SNR (dB) }\end{array}$ & $\begin{array}{l}\text { Predicted } \\
\text { Deviation in } \\
\text { Statistic }\end{array}$ \\
\hline 3.5 & $\begin{array}{l}\text { Phase } \\
\text { Difference } \\
\text { w/in Pulse }\end{array}$ & $1.8^{\circ}$ & $\begin{array}{l}85 \\
\text { (6 pulses) }\end{array}$ & 48 & $0.23^{\circ}$ \\
\hline 35 & $\begin{array}{l}\text { Bearing } \\
\text { Estimate } \\
\text { between } \\
\text { Pulses }\end{array}$ & $0.7^{\circ}$ & $\begin{array}{l}18 \\
\text { (18 pulses) }\end{array}$ & 38 & $0.22^{\circ}$ \\
\hline 125 & $\begin{array}{l}\text { Bearing } \\
\text { Estimate } \\
\text { Stability } \\
\text { Pulses }\end{array}$ & $1.3^{\circ}$ & $\begin{array}{l}7 \\
\text { (7 pulses) }\end{array}$ & 27 & $0.7^{\circ}$ \\
\hline
\end{tabular}

Although the data generally showed good correspondence to expected results, there seemed to be a slight systematic error. For all the tests, the slope of the line of measured bearing vs expected bearing was slightly greater than one - on average, it was 1.06 . The reason for this slope error is not clear, but there are a number of possible sources of error. The calibration used for the Hydrolab may have been inaccurate, leading to consistent miscalculation of the sound velocity. Also, the frequency of the pinger was measured in the field, not in lab, and the measured frequency may have been in error. This would lead to bearing offset errors of the type seen in Chapter 3 . 


\section{$\underline{5.3 \text { Short-Range Test Results }}$}

The short-range tests, carried out at a range of 3-4 meters, were designed primarily to prove that the USBL prototype would operate well in clearly understood acoustic conditions with high signal-to-noise ratios. Well-defined arrivals could be seen from the direct path and surface-bounce path, and the impact of the bottom-bounce arrival can be seen in the bearing estimates.

After the tests were carried out it was discovered that the hydrophone mount was interfering with the sound field. In general, bearing estimates from orientations in which the hydrophone array was pointing back under the barge (negative bearings, in the coordinate system used) were unreliable. For positive orientations the data are much better.

Test results for short-range tests on May 15 and May 21 are presented below. Only data from positive orientations are presented. These results were taken from the beginning section of each pulse, during which no multipath interference occurred. In the May 15 experiments the PVC pipe on which the hydrophone array was mounted was rotated by approximately $20^{\circ}$ between each measurement. On May 21 readings were taken at $10^{\circ}$ intervals. Due to the difficulty of precisely reading bearings from the hydrophone mounting used, bearing offset errors of several degrees can be expected. On May 14 the pinger was hanging unsupported in the water, and was moved back and forth by wave action. On May 21 the pinger was mounted on a PVC pipe to solve this problem, and the consistency of the results improved noticeably.

The May 21 results are shown in graph form in Fig. 5.29. The data show an very good fit to a line having a small y-intercept and slope of nearly one, indicating that no strong bias or unexpected effects are present in the data. The $1.2^{\circ}$ offset probably represents errors in accurately measuring the source-receiver geometry. As mentioned above, the phase measurements within each pulse were used to compute a standard deviation of $1.78^{\circ}$, measured over 6 pulses and 85 total acceptable measurements. 


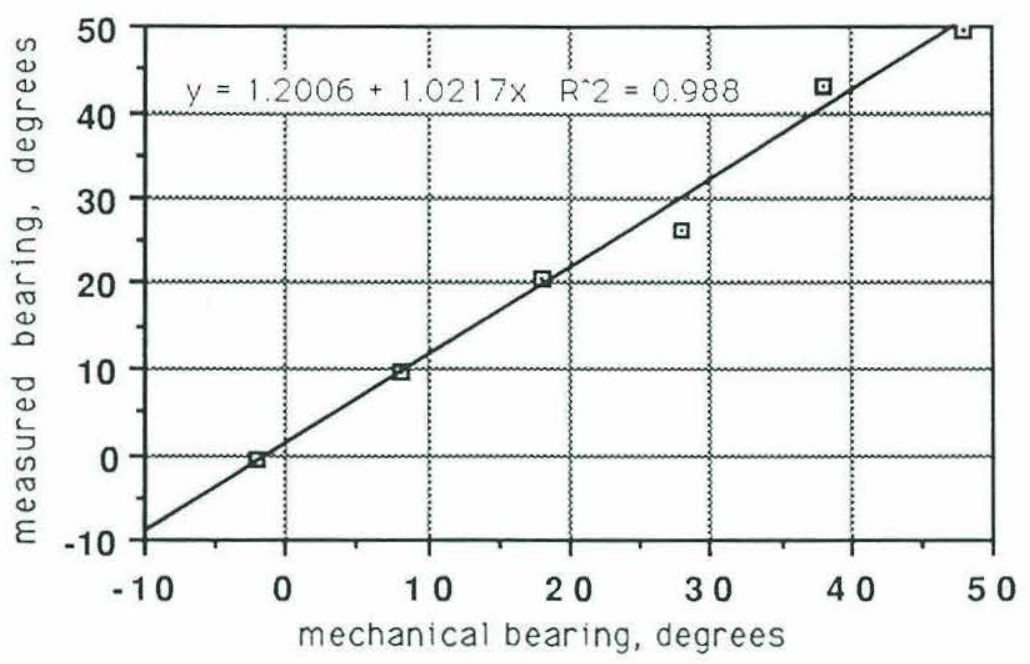

Figure 5.29: Bearing Estimation Results for May 21 Short-Range Tests

During the May 15 experiments the source and receiver were deployed at different depths. Thus bearing estimates made on the assumption they were coplanar were in error. These data were corrected for the out-of-plane arrivals by estimating the angle from the horizontal $\varphi$ from the measured source-receiver and depth difference. This angle was substituted into the generalized USBL measurement formulas, Eqns. (2.3) and.(2.4) from Chapter 2, and the correct bearing angle $\theta$ was calculated. Figure 5.30 below shows the effect of this correction. The initial bearing estimates are indicated by circles, and the corrected estimates are shown by diamonds. As can be seen, the correction moves the results closer to the expected answer, shown by the line below. 


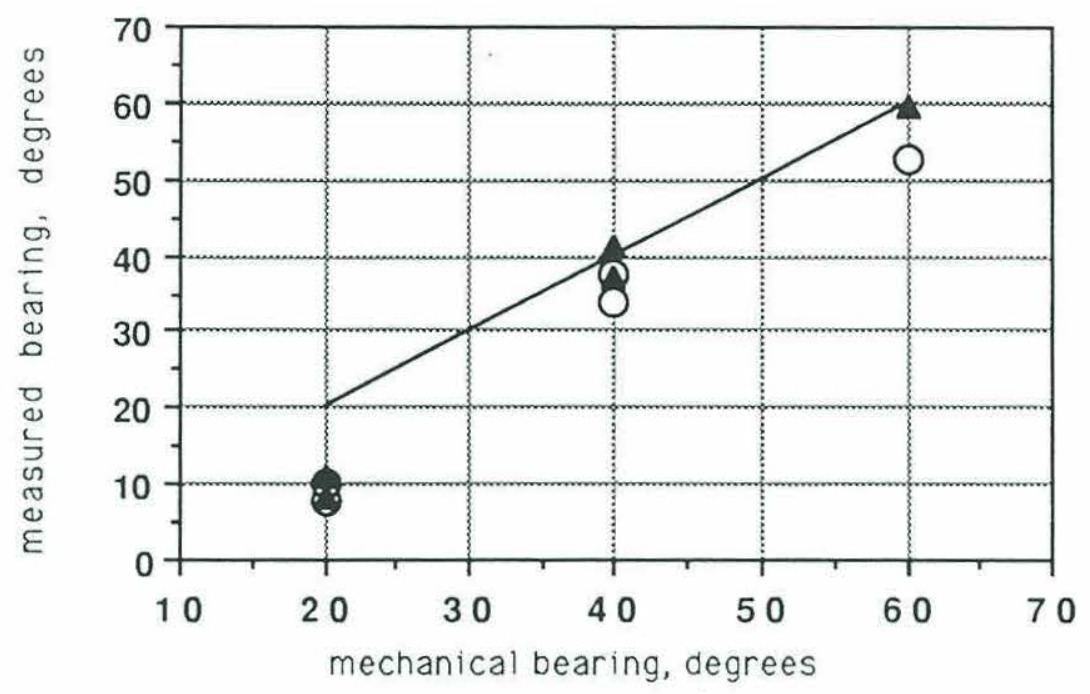

Figure 5.30 : Effects of Out-of-Plane Correction on May 15 Results line $=$ expected bearing, circle $=$ raw bearing measurement, diamond $=$ corrected measurement

Although the figures above provide a useful means of quantifying system performance, much more can be learned by looking at the actual field results. Figure 5.36 at the end of the chapter shows some interesting results from May 21. Each of these plots shows the calculated signal power (circles) and estimated bearing (plus signs) at many times within a single pulse.

The effects of multipath arrivals can be seen clearly in these plots. The signal power generally takes a jump approximately $2.5-3 \mathrm{msec}$. after the beginning of the pulse. This time delay agrees well with the predicted delay, shown in Table 4.1. It can be observed that the size of the power jump changes from pulse to pulse. As the surface of the water moves up and down, the path differences between the direct and surface-reflected paths change by a few centimeters. This leads to varying amounts of constructive or destructive interference. This is an example of the Lloyd's mirror effect, in which a sound source near the air-water interface will create an image source above the water which is 
equal in power but opposite in sign. As the surface of the water moves, the lobes and nulls of the resulting beam pattern move across the receiving hydrophone, leading to the power fluctuations seen.

A third arrival can be seen affecting the bearing estimate at about $7.5 \mathrm{msec}$ after the beginning of the pulse, although no appreciable rise in the power level is seen. This is presumably the bottom return, which can be expected to have a small amplitude due to absorption of sound by the muddy bottom.

An interesting phenomenon associated with the impulse response of the pinger is seen in the data. At the beginning of each pulse the pinger takes a few cycles to settle into transmitting a steady tone. This is seen on a number of the plots below, for example Fig. 5.36a. The power is quite low at first, then increases to a peak value before slowly dropping off. The transients generated by this ringing cause errors in the bearing estimate, as seen in the data. The Datasonics transducer element used was designed to resonate with a Q of 5. This does not include the effects of the resonance in the driver circuit, so the Q for the whole pinger may be somewhat different. As a rule of thumb the number of transducer ring-up cycles is equal to the transducer Q. Thus a $\mathrm{Q}$ on the order of 5 agrees fairly well with the May 21 data, in which each data point represents results from 4 cycles of sinewave and most often only 1 point shows the transient effects.

As mentioned earlier, the effects of interference on phase difference estimation are calculated in Appendix C. The calculation assumes that two paths are present, the direct path and a surface-reflected path. The results predict that the amplitude of the wave formed by interference depends on both the path length difference and the angle from the horizontal $\varphi$ at which the surface-reflection arrives at the hydrophones. The phase difference, however, should depend only on $\varphi$. An examination of the test data shows that the changes in bearing are not stable. If the calculation in Appendix $\mathrm{C}$ is to believed, then, the angle $\varphi$ must be changing from pulse to pulse as water waves tilt the water surface back and forth. 


\subsection{Medium-Range Tests}

Tests at ranges of 35 meters were carried out with the pinger mounted at one end of the Navy Yard barge and the hydrophones mounted at the other. These tests were designed to gauge USBL performance in a more realistic scenario.

Some typical test results for the 35 meter range are shown in Fig. 5.37 at the end of the chapter. The results from tests carried out on June 26 and July 6 are summarized in Fig. 5.31. Bearings estimated from received pulses are plotted against the nominal, or expected, bearing. This figure shows that the measured and estimated bearings are in quite close agreement over the range of measurement, although the offset in slope mentioned above is present. The data show an excellent fit to a line having a y-intercept close to zero and slope of nearly one. The fact that the y-intercept is smaller than for the short-range data probably reflects increased experience in setting up and measuring the test apparatus accurately, rather than actual improved system performance at the longer range.

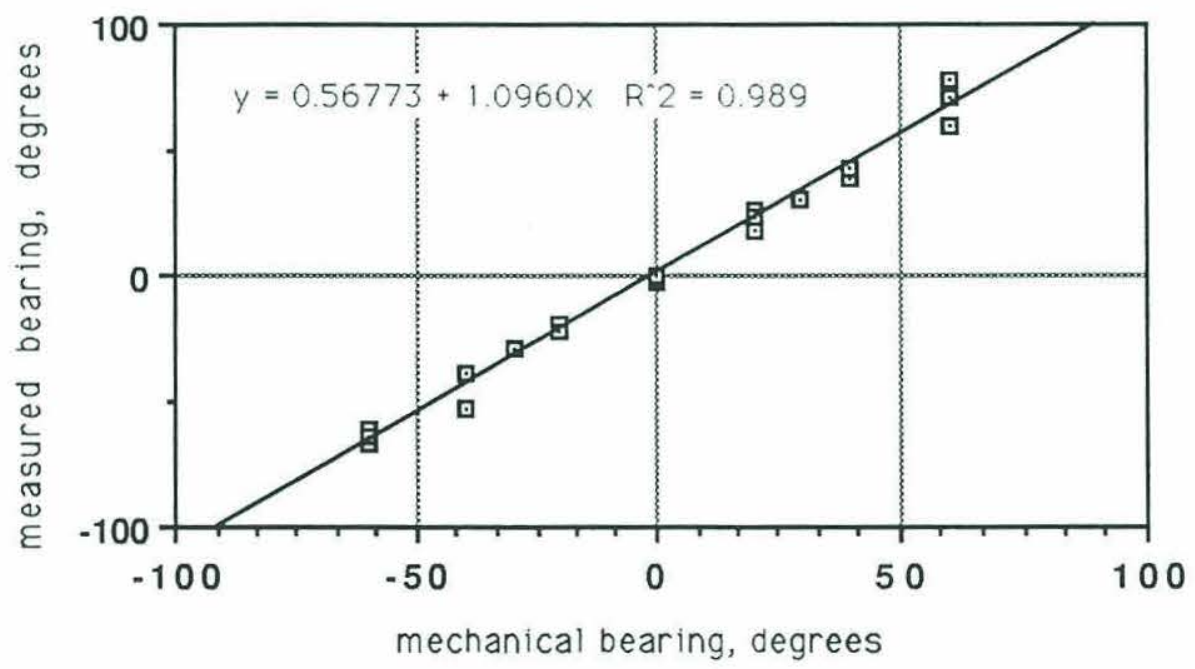

Figure 5.31: Bearing Measurement Results for Medium-Range Tests 
Tests were carried out on July 8 to see how stable the results obtained during periods of multipath interference were. An additional set of tests were carried out to study the effect of pinger orientation. A large number of pulses were recorded at a bearing of $0^{\circ}$. For half of the data the pinger was oriented facing down, and the rest of the data was taken with the pinger facing the surface. Figure 5.32 below shows the mean and standard deviation of the signal power and bearing when the pinger was oriented down, averaged over 11 pulses, and Fig. 5.33 shows results from 7 pulses recorded when the pinger was facing the surface. For both orientations the standard deviation of bearing for the uncontaminated signal at the beginning of the pulse can be seen to be quite small $\left(<1^{0}\right)$, while the deviation for the remainder of the pulse can be quite high. The large changes in the effects of interference on the bearing estimate are probably due to the motion of the barge relative to the bottom and vibration of the PVC pipes supporting the pinger and hydrophones. The larger changes for the bottom-facing orientation might be expected, as the movement relative to the bottom will be more important for these tests.

A number of multipath features can be seen from the mean values. An attempt was made to identify some of these. The expected arrival times of the reflections from the bottom of the barge (assuming $1 \mathrm{~m}$. draft), the bottom, and the pier wall at the side of the barge shown in Table 4.1 are noted on the plots below. The calculation for the expected arrival time for the wall bounce seems to somewhat off, but is close enough to allow identification of the $2 \mathrm{msec}$. arrival. It is interesting to note that the signal power levels for the surface-facing orientation are higher than for the bottom-facing tests. This is reasonable, as the reflection off the barge bottom can be expected to be much stronger than the bottom reflection, so the direct and reflected paths will add to give a larger power. As a result, the wall arrival is somewhat masked in the surface-facing data. 


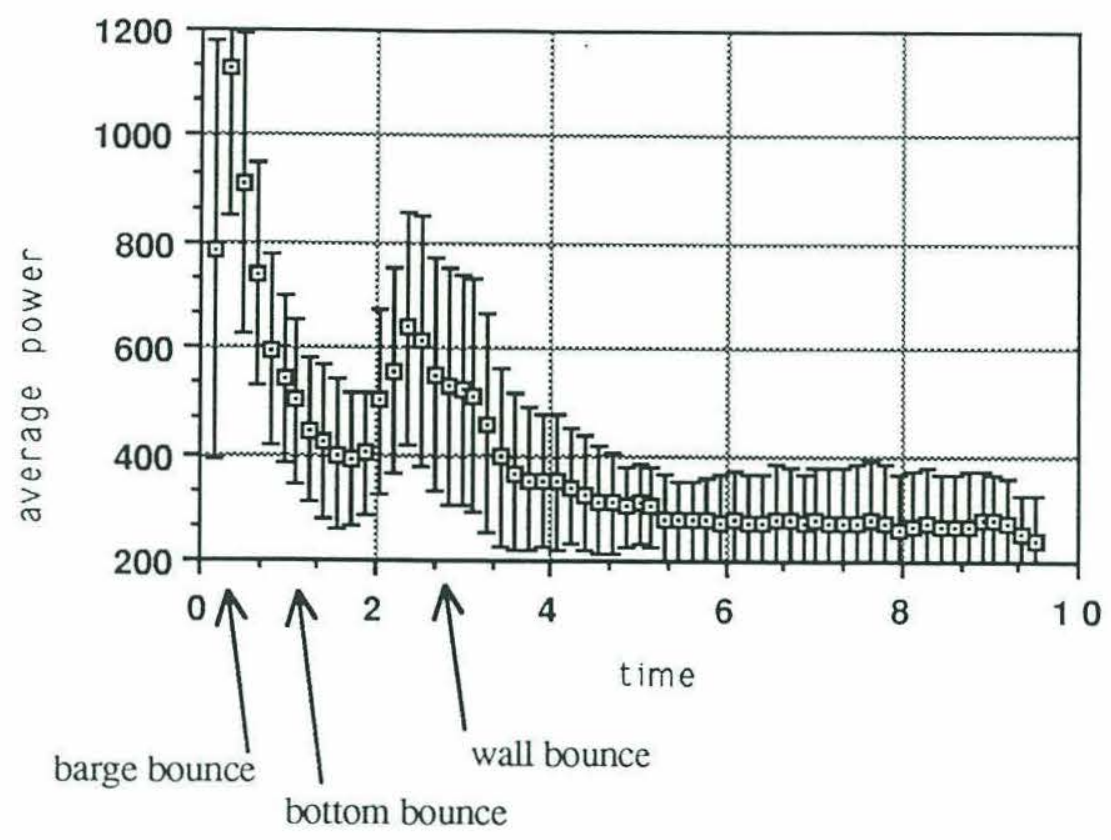

Figure 5.32: Results for Bottom-Facing Pinger Orientation a) Mean Power Throughout Pulse

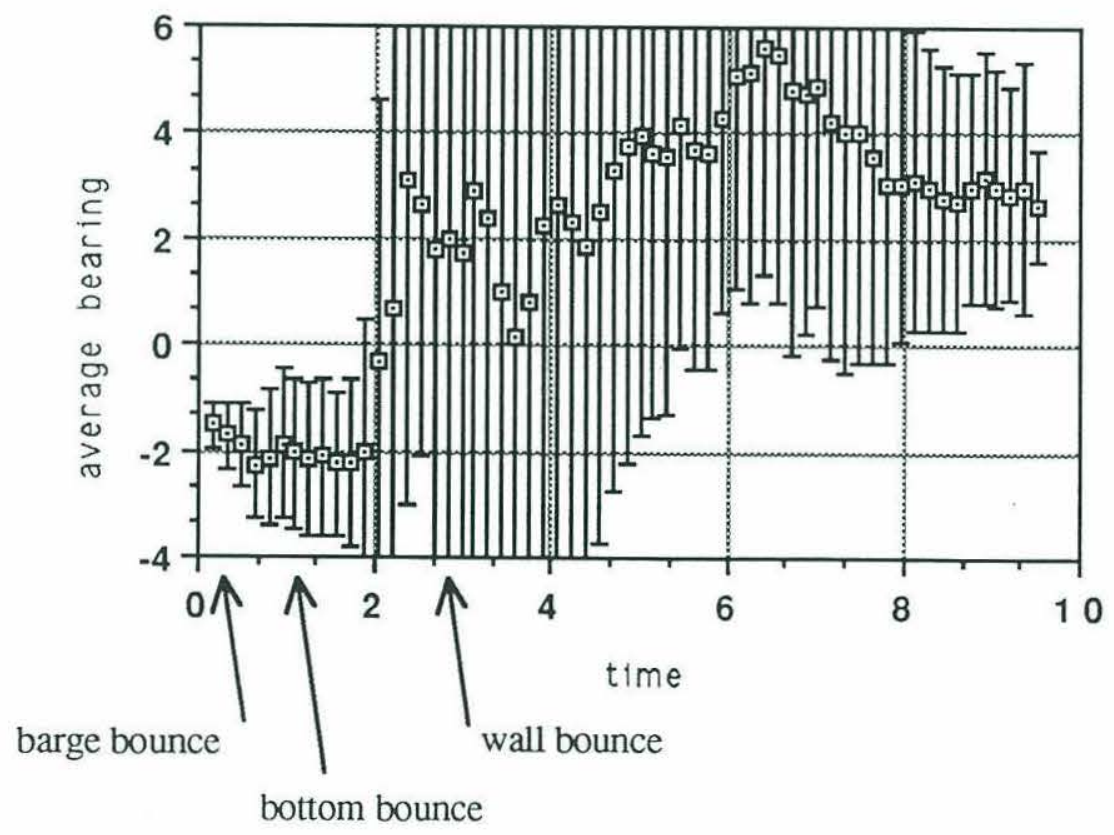

b) Mean Bearing Throughout Pulse 


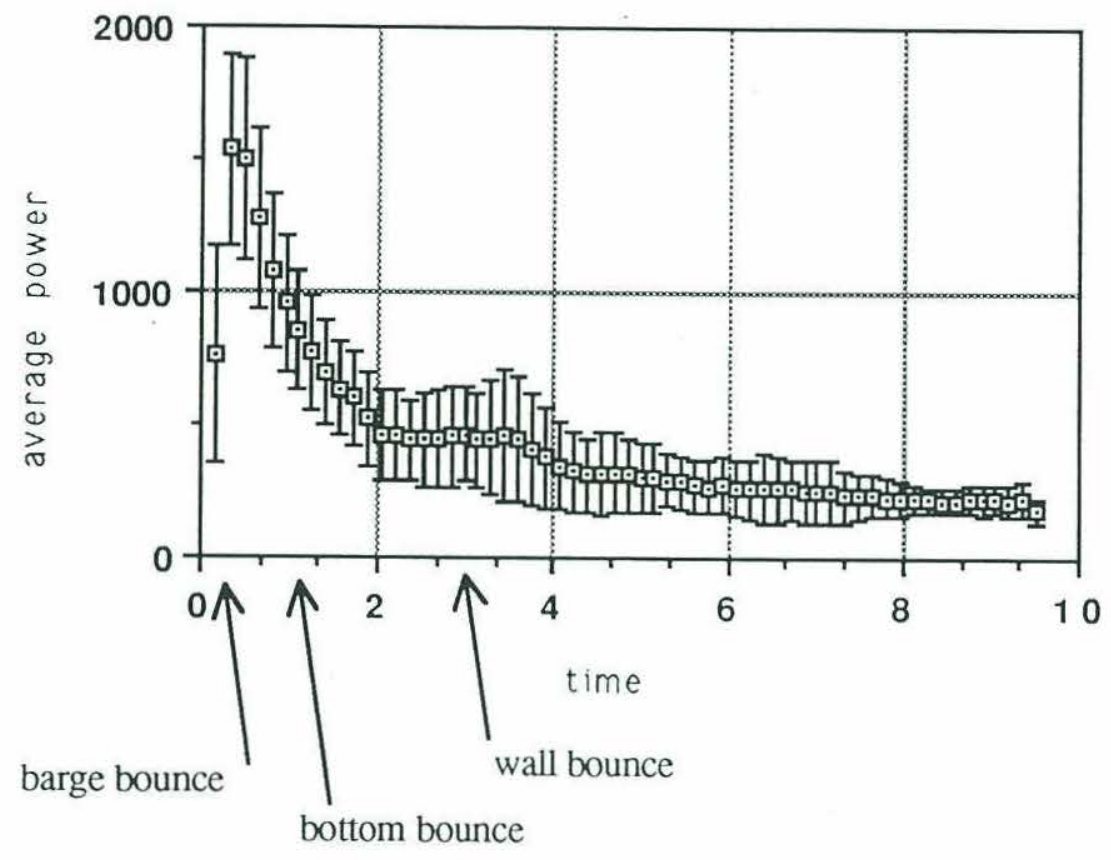

Figure 5.33: Results for Surface-Facing Pinger Orientation a) Mean Power Throughout Pulse

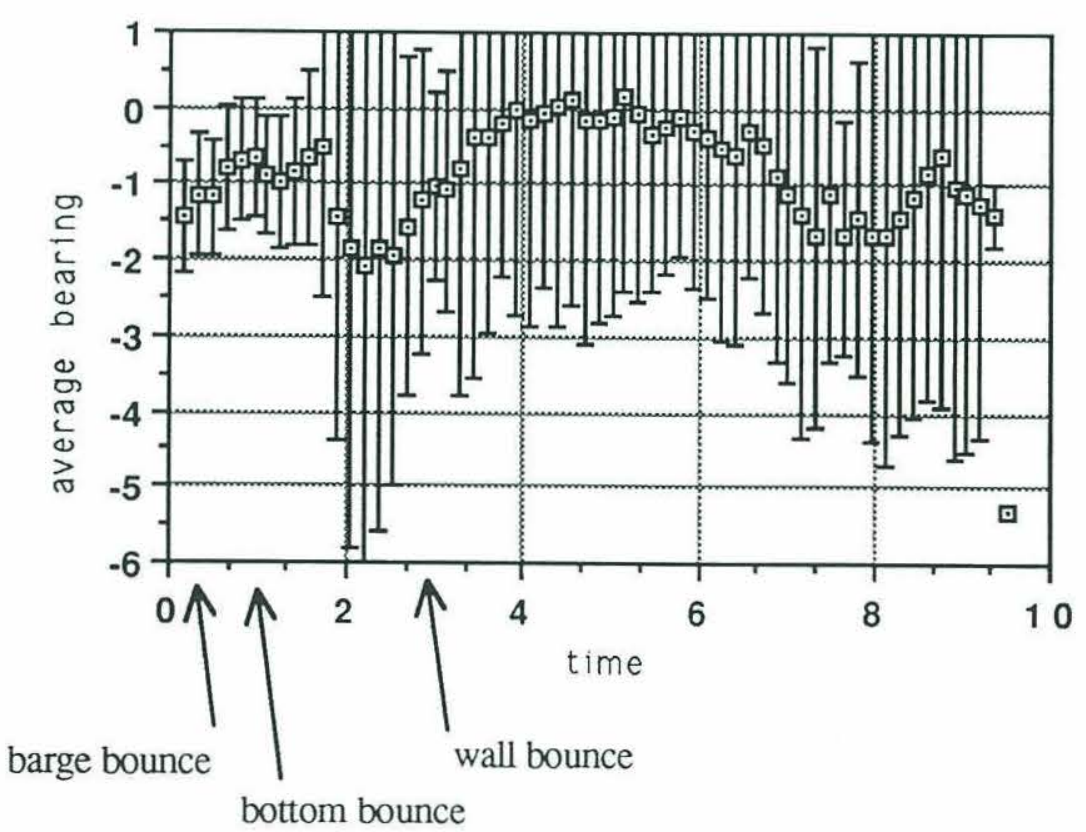

b) Mean Bearing Throughout Pulse 


\section{$\underline{5.5 \text { Long-Range Tests }}$}

A number of tests were carried out in June and July to estimate bearing over the 125 meter source-receiver separation. Many test setup and hardware problems were encountered in carrying out these tests, and only the data from the last set of tests are presented. During testing the sound speed profile was measured, and a ray-trace program was used to calculate expected arrivals for the measured sound speed profile. The results of the ray-trace as seen in Table 4.1. The ray-trace predictions show that approximately 5 cycles, or slightly more than one integration period, of uncontaminated direct path should be present. The effects of interference after the first few cycles are seen clearly in Fig. 5.37, which shows some representative received pulses.

The bearing estimates from the first four cycles of each pulse are shown below in Fig. 5.34. The agreement between expected and measured bearings is worse than for the medium and short range tests. This loss in consistency can largely be explained by the drop in signal strength, as discussed in section 5.2 above.

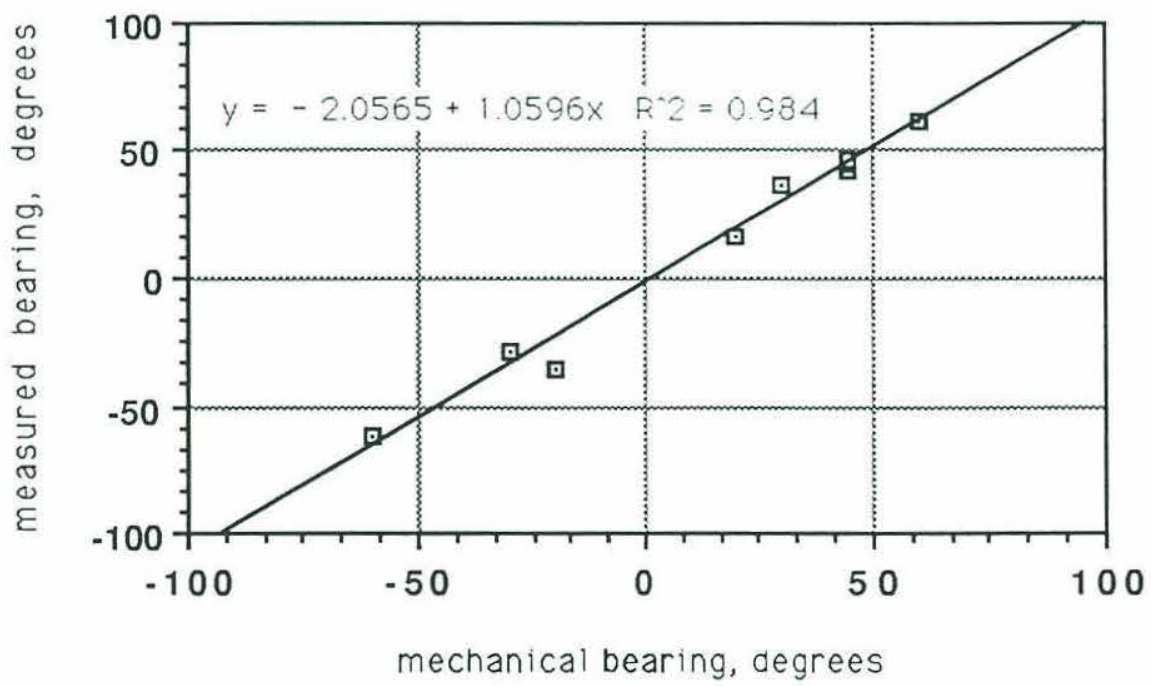

Figure 5.34: Bearing Estimation Results for Long-Range Test Results 
In order to determine the stability of the measurement over the 125 meter range, 7 pulses were recorded at a bearing of $45^{\circ}$. Of these, 2 were not recorded in full as the pulse power fell below the detection threshold shortly after triggering. The averaged power and estimated bearing for the remaining pulses are shown in Fig. 5.35 below. The number of pulses recorded is not really sufficient to make strong statistical statements, but the plots give some indication of what is happening.

Figure 5.35 shows interesting differences from the power and bearing plots generated from the medium-range data. The received signals for two of the pulses extended beyond the $9.5 \mathrm{msec}$ duration of the transmitted pulse, showing the increased effects of reverberation at longer range. The power at the beginning of the pulse, although highly variable, seems to fall off less rapidly in the first millisecond or so than was the case for medium-range data. This can be explained, as multiple ray paths are arriving during this period (including all those shown in the ray-trace plot in Chapter 4).

A number of noticeable arrivals are seen. An arrival is seen at $2 \mathrm{msec}$ which has a strong impact on the bearing estimates. This arrival may correspond to a path predicted by the ray-trace program to arrive $2.1 \mathrm{msec}$. after the direct pulse, after undergoing 2 surface bounces and 1 bottom bounce. This ray arrives at an vertical angle of $12.5^{\circ}$. This angle is the largest arrival angle of any ray which does not bounce off the bottom more than once (rays which interact with the bottom several times will be severely attenuated), so it would be expected to cause the largest errors in bearing. Several extremely strong arrivals are seen around 7-9 msec. The strength and arrival time of these arrivals do not correspond to any of the paths predicted by the ray-trace, so it seems reasonable to assume they represent reflections of the direct path off the pier at which the barge is moored.

One of the most interesting facts observed from Fig. 35b is that the bearing estimate is fairly stable for the first $2 \mathrm{msec}$, although multiple arrivals are interfering during this time period. This suggests that periods of overlapped multipaths may actually be usable for 
bearing estimation, as long as the vertical arrival angles of the rays are close to that of the direct path. In Appendix $\mathrm{C}$ the expected effects on phase of interference between a direct and a surface-reflected arrival are calculated. It is shown there that if $\varphi$, the vertical arrival angle of the surface-reflected pulse, is known, then the true (horizontal) bearing can be calculated from

$$
\theta=\operatorname{Arcsin}\left(\frac{2 \Delta \phi_{y}}{\pi(1+\sin \varphi)}\right)
$$

where $\varphi=90^{\circ}$ corresponds to an in-plane arrival. For the bearing calculations shown in Fig. 5.35 it was assumed that all arrivals were in the plane of the array, and the apparent bearing was calculated from

$$
\theta=\operatorname{Arcsin}\left(\frac{\Delta \phi_{y}}{\pi}\right)
$$

It can be seen from Eq. (5.1) that if the arrival is close to in-plane, the errors introduced by calculated bearing from Eq. (5.2) will be quite small, as seen in the data. An attempt was made to use Eq. (5.1) and predicted arrival angles from Table 4.1 to extract corrected bearing information from Figure 5.35b. A small correction was apparent, but was much too small to account for the changes in apparent bearing seen in the data. This suggests that the hydrophone pair may have been tilted in the water at some angle to the water surface, or that the predicted arrival angles may not have been accurate. 


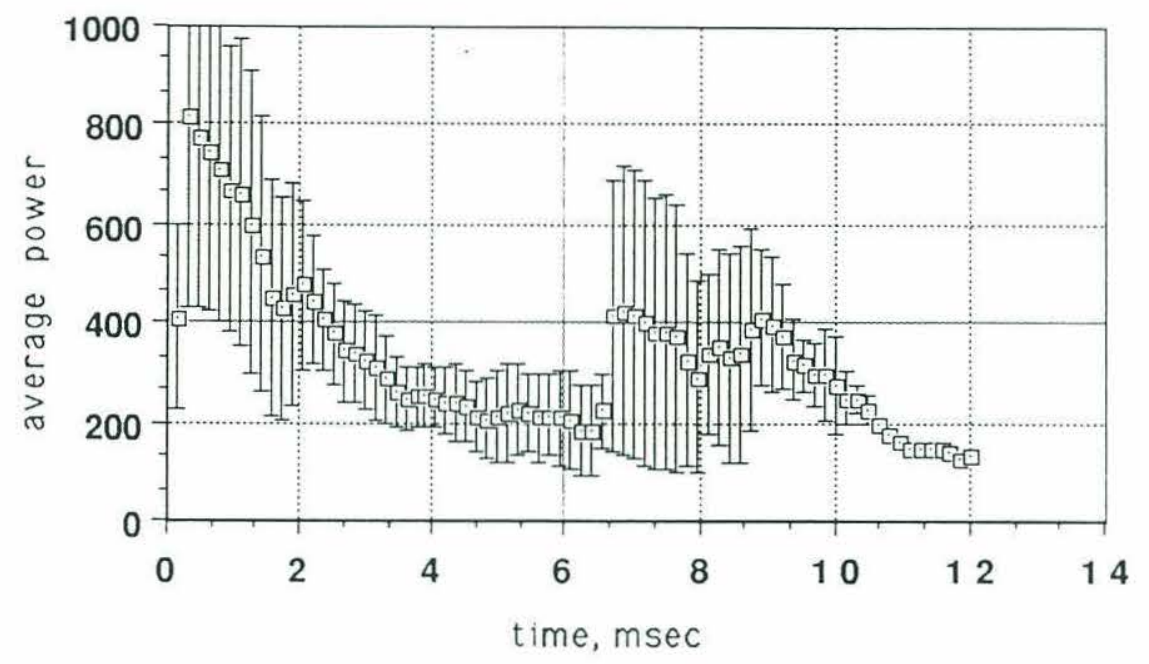

Figure 5.35: Results for 125 Meter Range at $45^{\circ}$ Bearing a) Average Signal Power for 5 Pulses

2 pulses extend to $12 \mathrm{msec}$ duration; others end after approx. $9 \mathrm{msec}$.

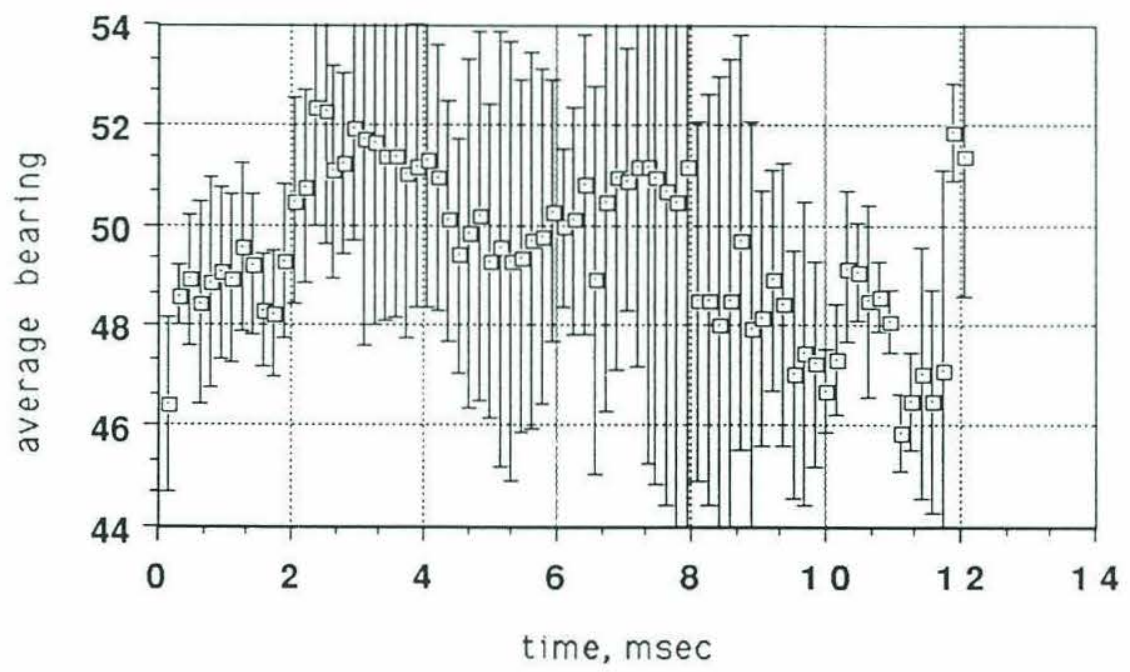

b) Average Measured Bearing vs Time 


\subsection{Correlation between Fluctuations in Signal Power and Bearing}

Many of the received pulses shown at the end of the chapter seem to display a correlation between the estimated bearing and the signal power. A correlation was expected, as the multipath interference which modulates the signal power also affects the phase difference estimate. An attempt was made to determine how statistically significant this apparent correlation was. The exponential decay of pulse strength due to capacitor discharge was subtracted from the signal power data, and correlations between power and bearing were calculated. In most of the pulses studied, this correlation was found to be quite small when measured throughout the whole pulse. When the correlations during sections when strong multipath features were present were calculated, however, the correlations rose significantly. Results for the 5 pulses studied are shown below. In general a multipath arrival which gives rise to a noticeable change in the signal power will also cause a change in the estimated bearing, but some multipath arrivals can be identified which cause changes in the bearing estimate only. Thus the bearing estimate is more sensitive than the signal power to multipath interference.

Table 5.2: Calculated Correlations between Power, Bearing Fluctuations in 5 Pulses

\begin{tabular}{|lllll|}
\hline Range (meters) & $\begin{array}{l}\text { Correlation } \\
\text { Coefficient for } \\
\text { Entire Pulse }\end{array}$ & $\begin{array}{l}\text { Subsection of } \\
\text { Pulse (msec) }\end{array}$ & $\begin{array}{l}\text { Multipath } \\
\text { Feature }\end{array}$ & $\begin{array}{l}\text { Correlation } \\
\text { Coefficient for } \\
\text { Expanded } \\
\text { Section }\end{array}$ \\
\hline 3.5 & 0.36 & $2-6$ & surface bounce & 0.96 \\
3.5 & 0.21 & $2-6$ & surface bounce & 0.71 \\
35 & 0.20 & $0-4$ & wall bounce & 0.67 \\
35 & 0.37 & $0-4$ & $\begin{array}{l}\text { wall bounce } \\
\text { ray, wall } \\
\text { arrivals }\end{array}$ & 0.57 \\
125 & 0.60 & $2-8.5$ & & 0.78 \\
\hline
\end{tabular}


Figure 5.36: May 21 Test results $+=$ bearing estimate

$\mathrm{o}=$ narrowband (signal) power

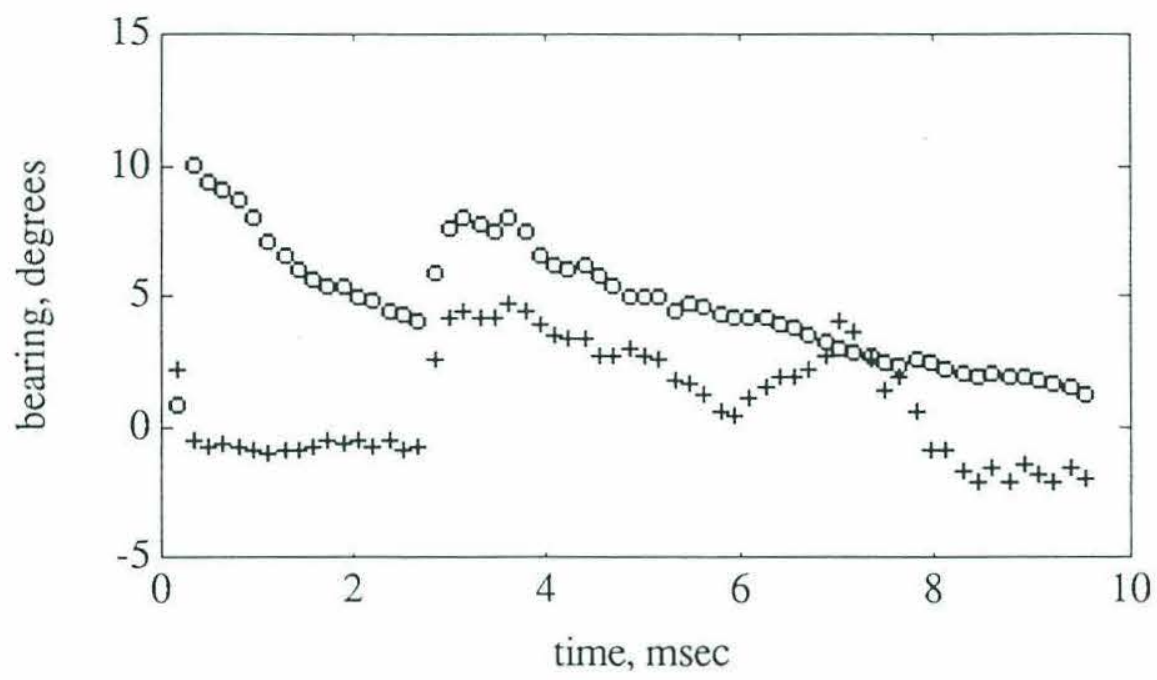

a) -2 degrees; narrowband power scaled by 100

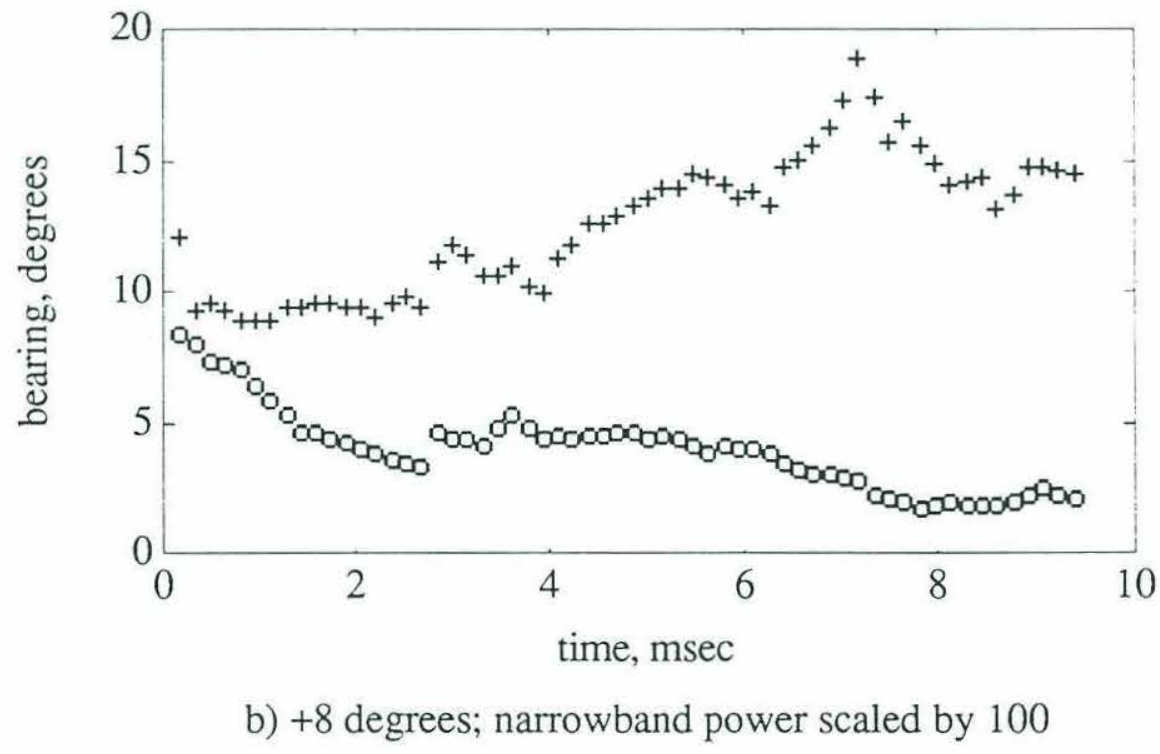




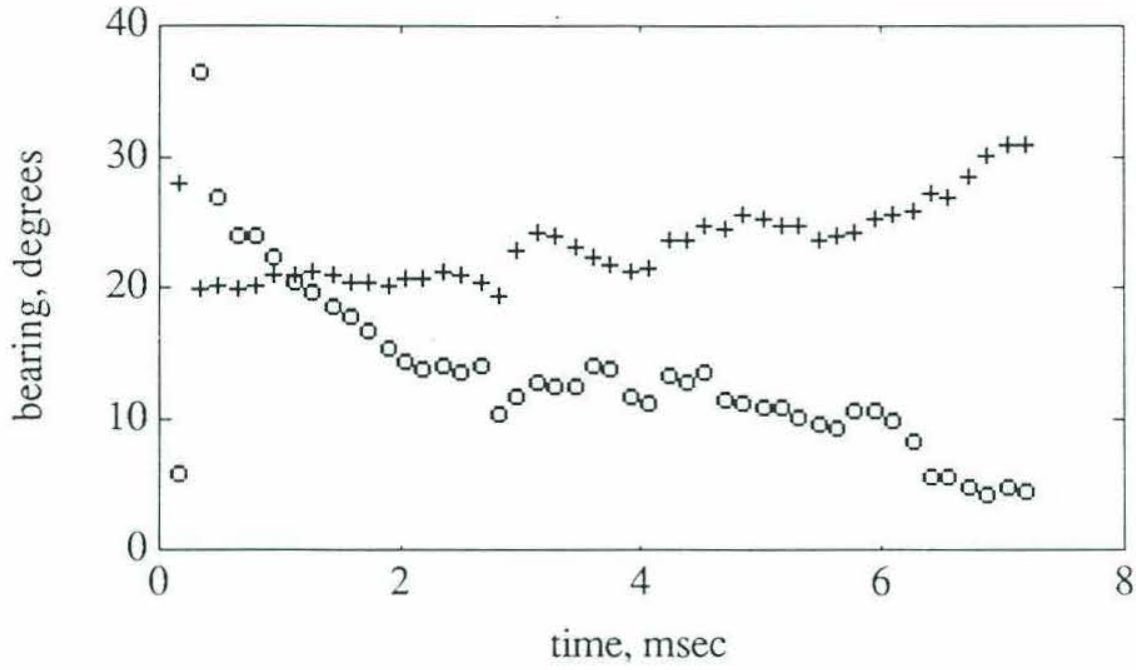

c) +18 degrees; narrowband power scaled by 20

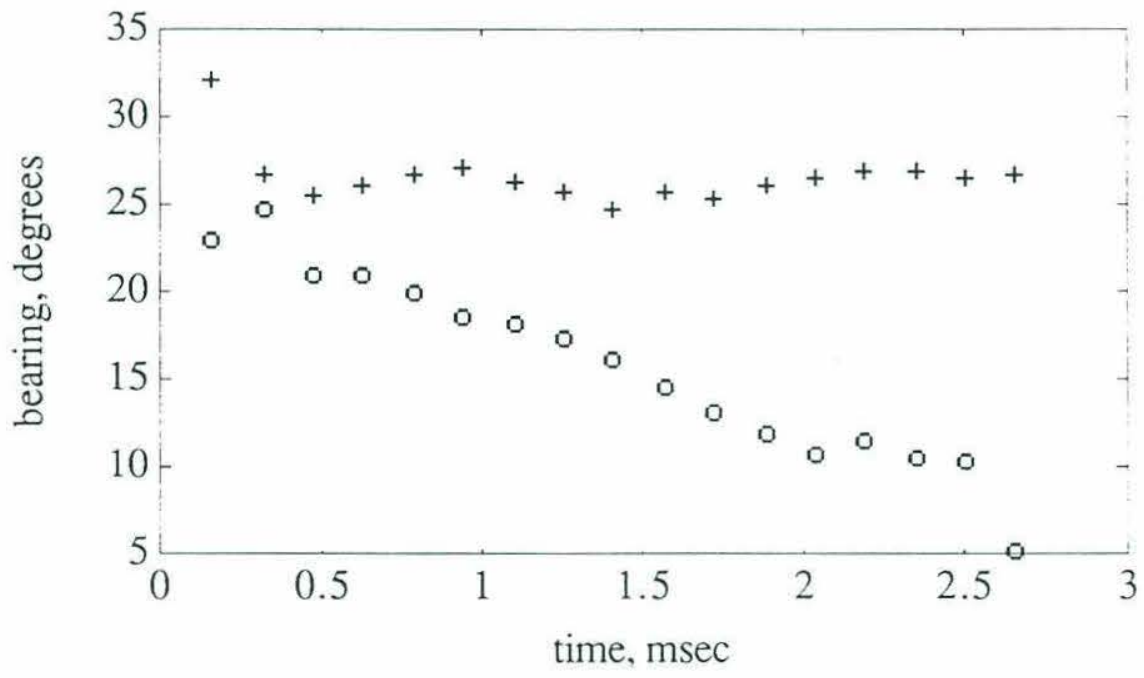

d) +28 degrees; narrowband power scaled by 20 


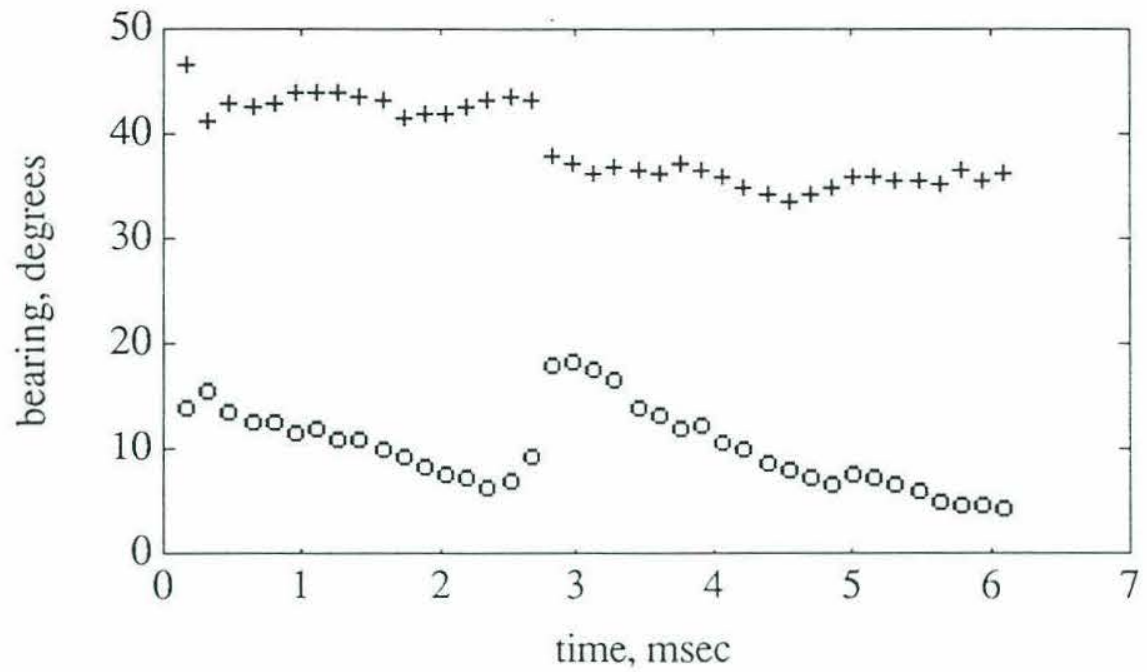

e) +38 degrees; narrowband power scaled by 20

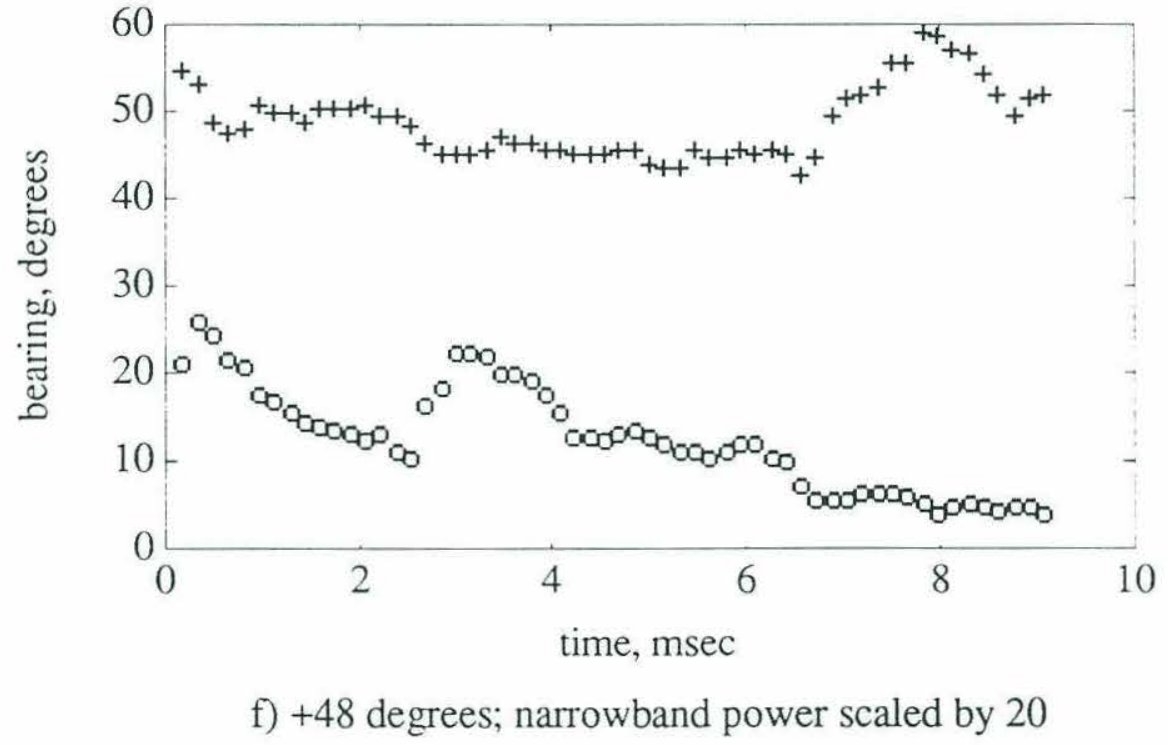



Figure 5.37: Test Results for 35 Meter Range $+=$ bearing estimate $\mathrm{o}=$ narrowband (signal) power

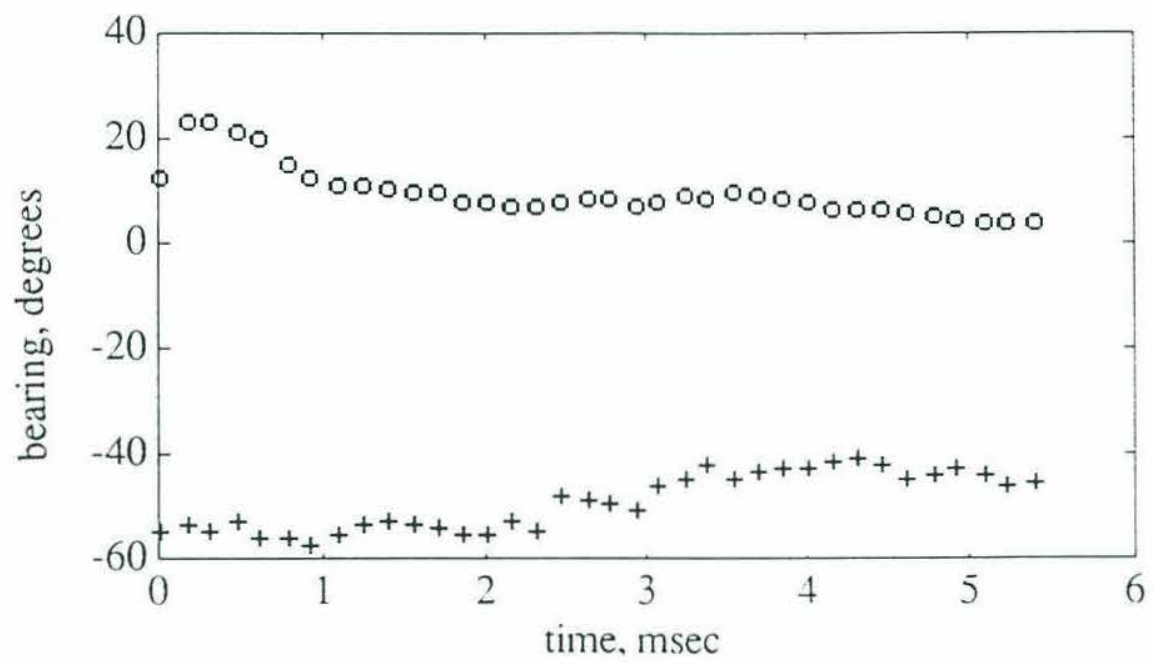

a) -60 degrees; narrowband power scaled by 100

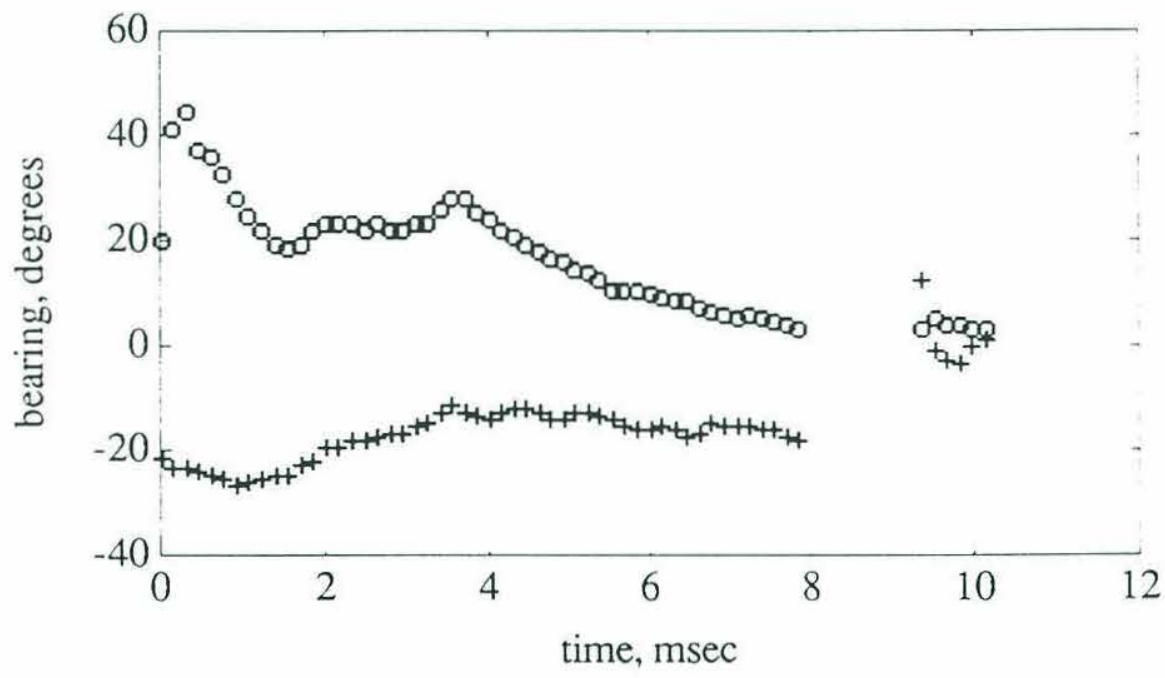

b) -30 degrees; narrowband power scaled by 100 


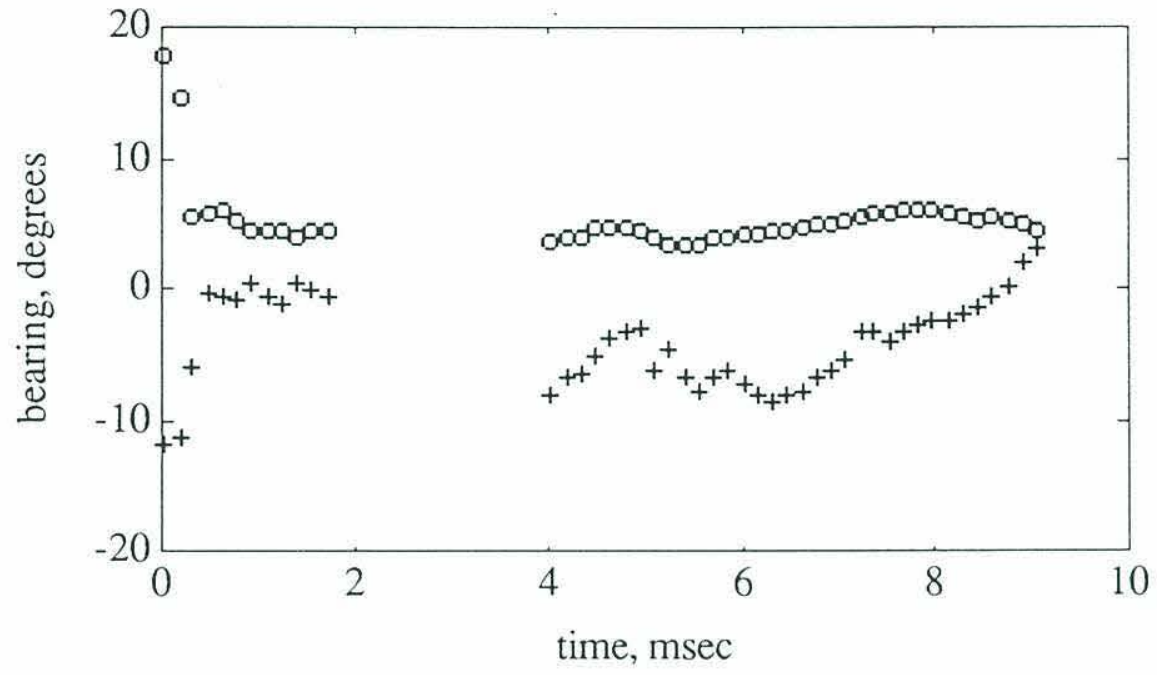

c) -20 degrees; narrowband power scaled by 100

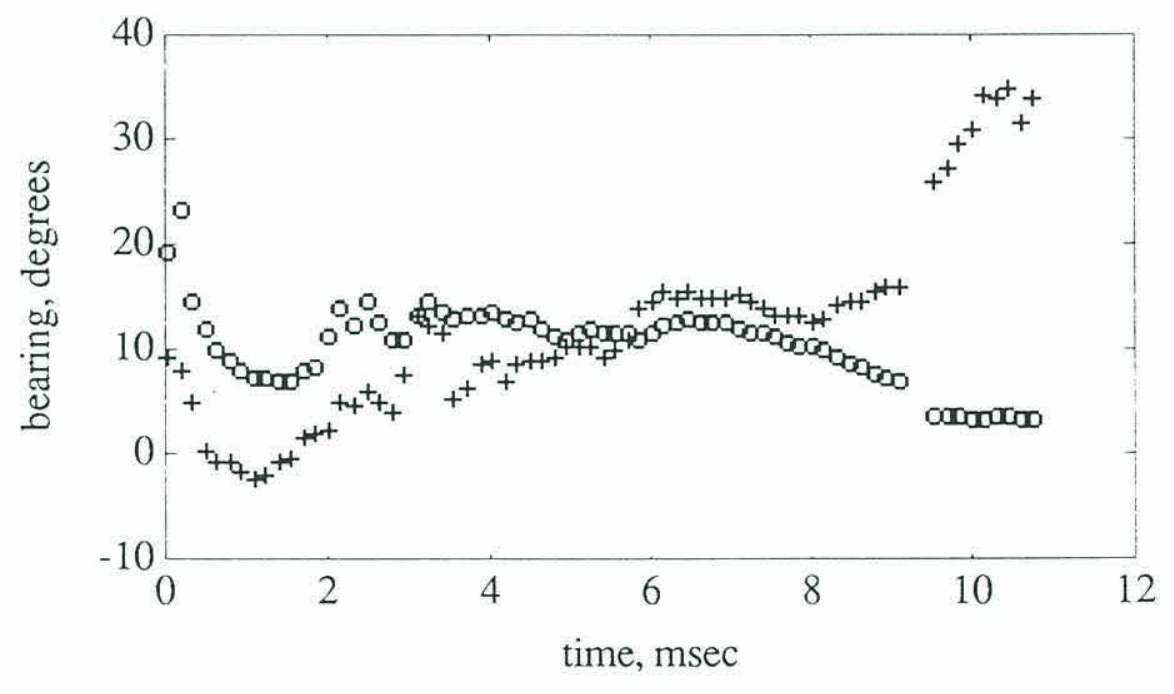

d) 0 degrees; narrowband power scaled by 100 


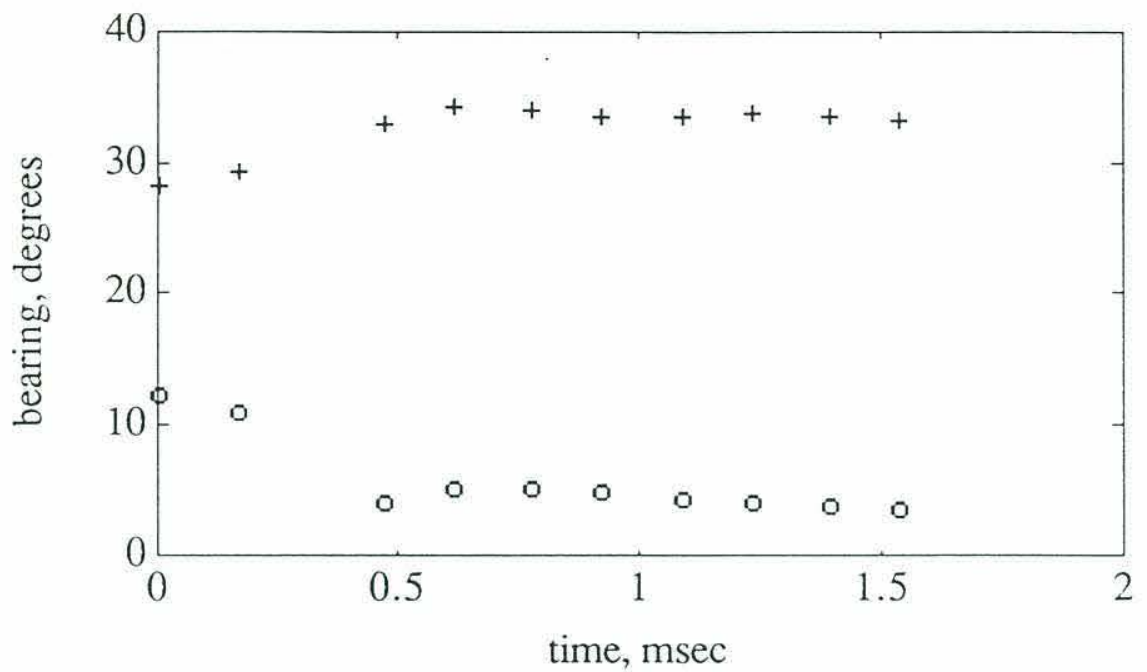

e) 20 degrees; narrowband power scaled by 100

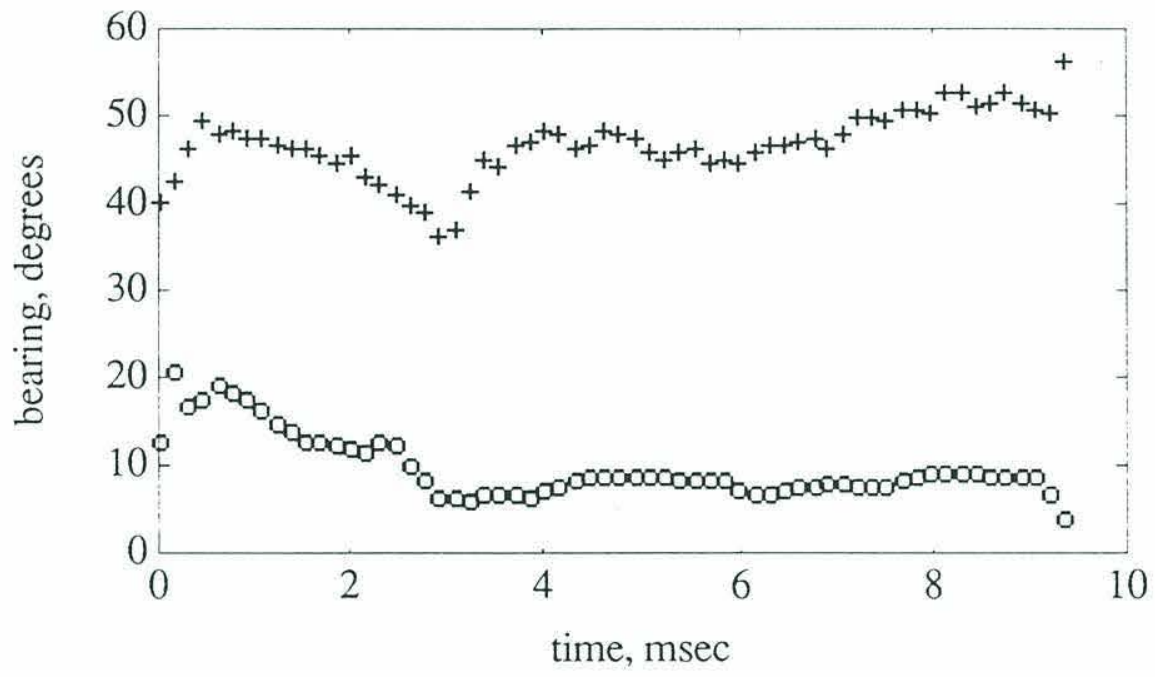

f) 30 degrees; narrowband power scaled by 100 


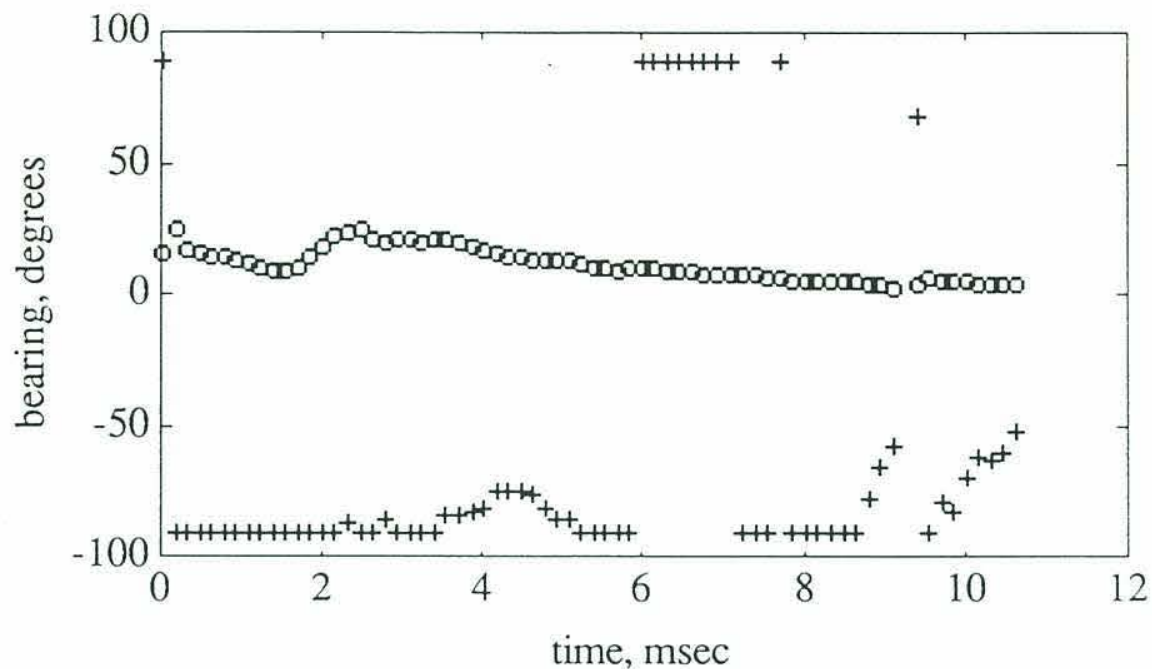

h) 60 degrees, narrowband power scaled by 100 
Figure 5.38: July 6 Long-Range Test Results $+=$ bearing estimate

$\mathrm{o}=$ narrowband (signal) power

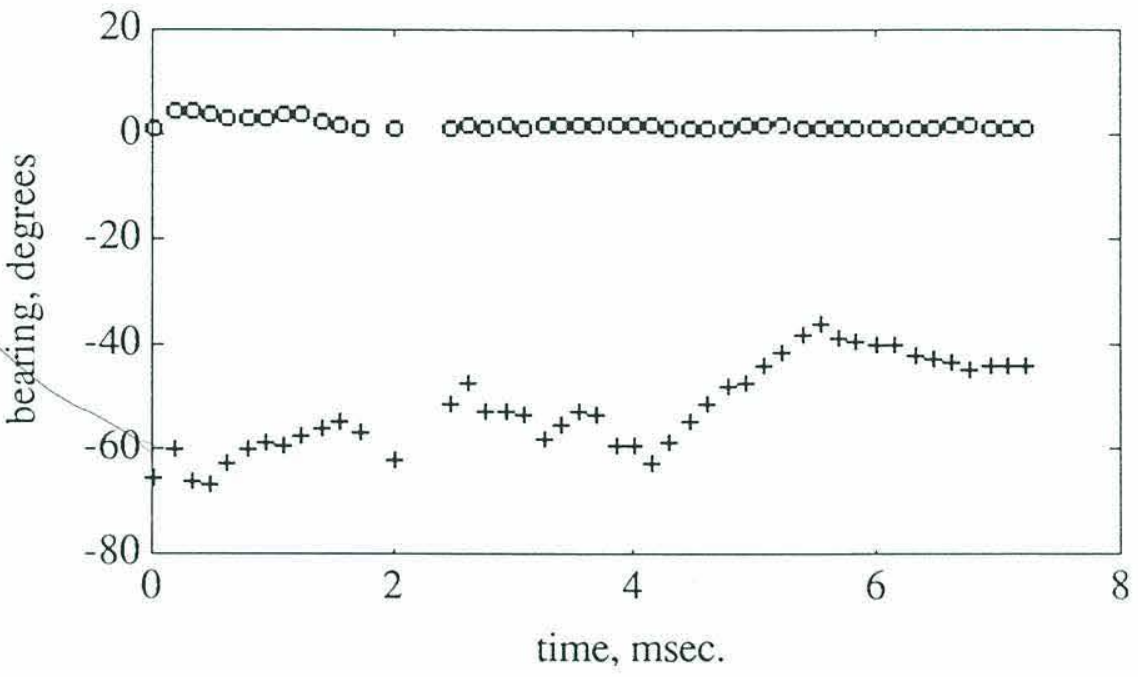

a) $-60^{\circ}$; signal power scaled by 100

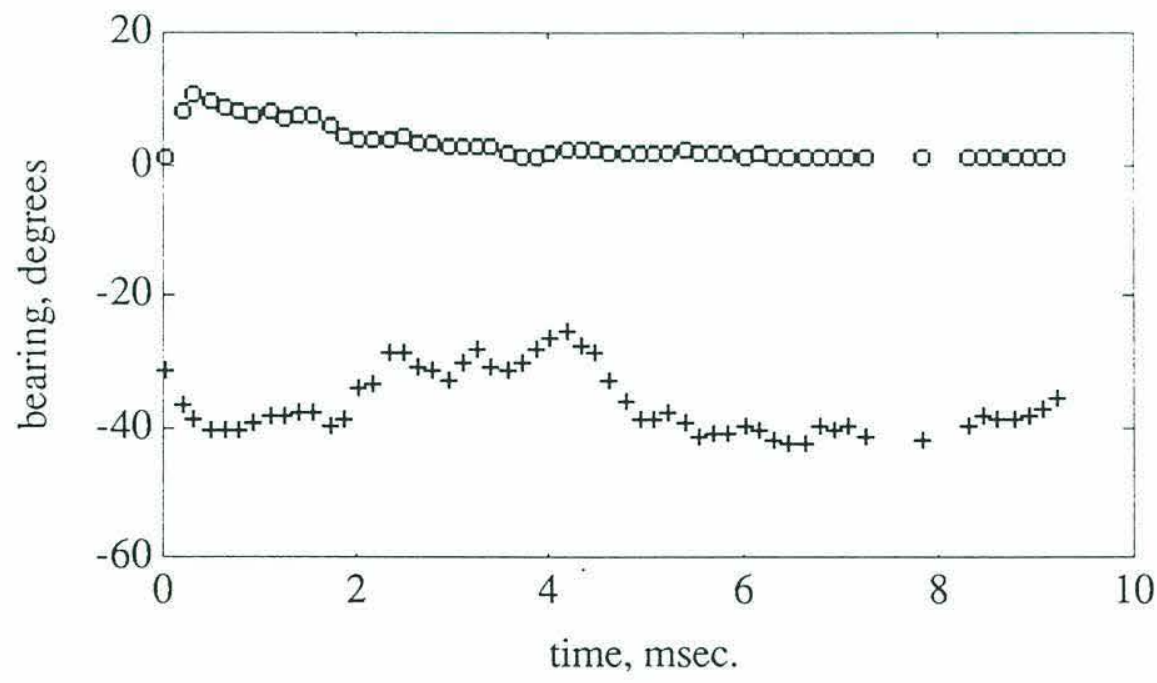

b) $-40^{\circ}$; signal power scaled by 100 


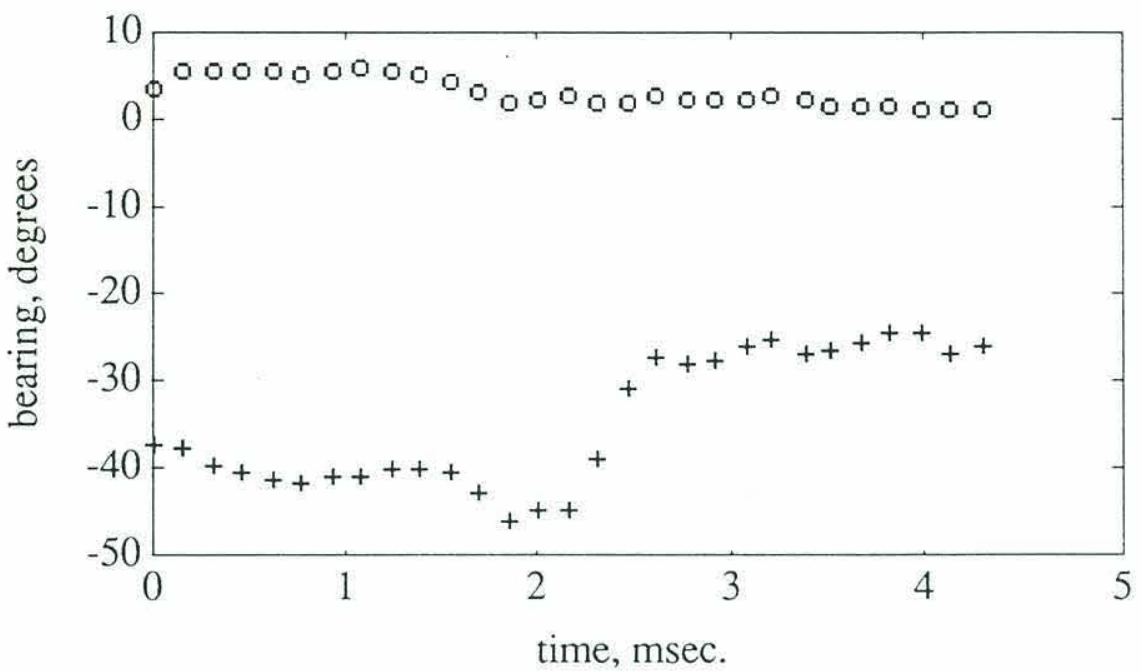

c) $-20^{\circ}$; signal power scaled by 100

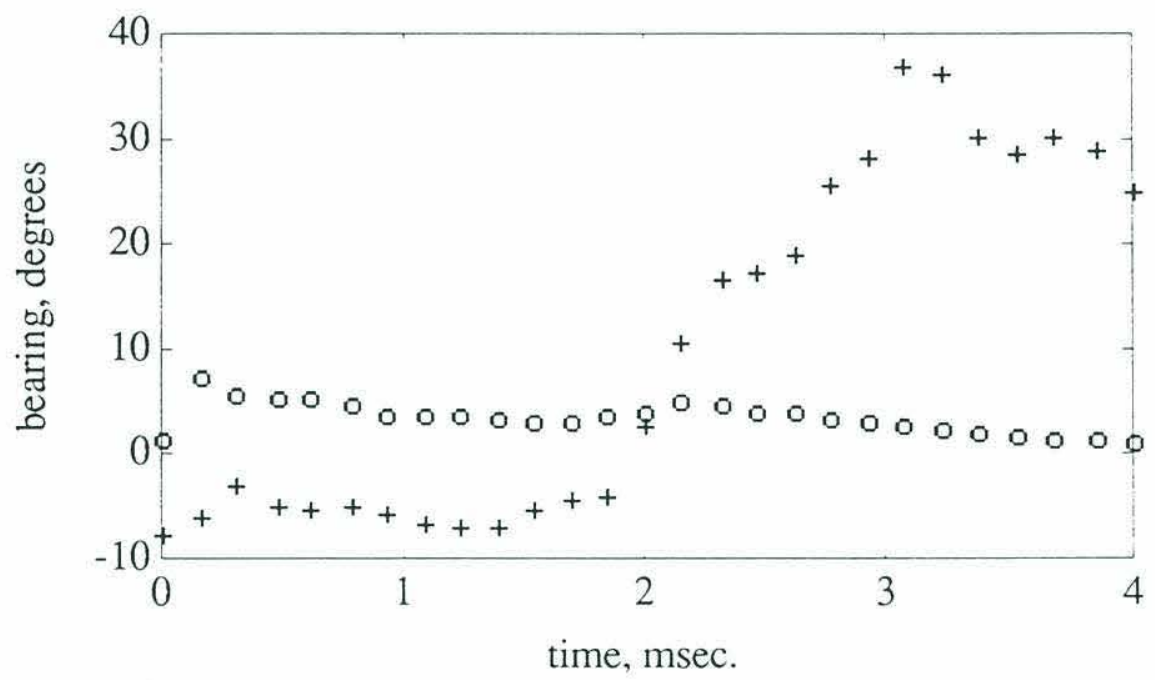

d) $0^{\circ}$; signal power scaled by 100 


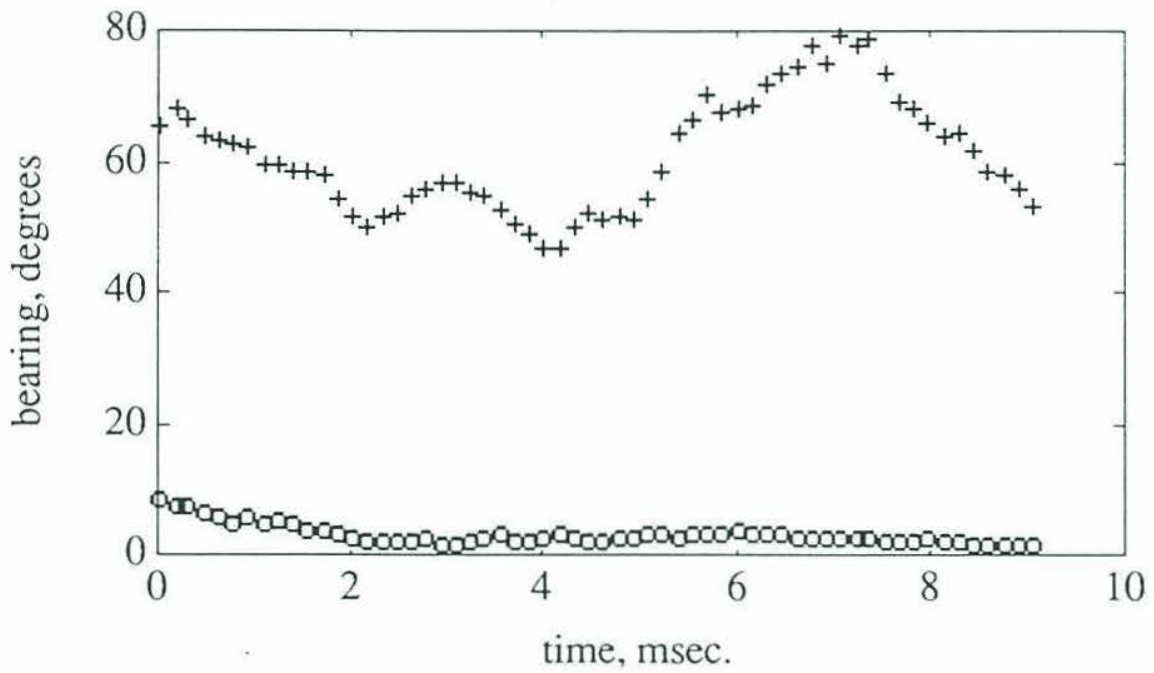

e) $60^{\circ}$; signal power scaled by 100

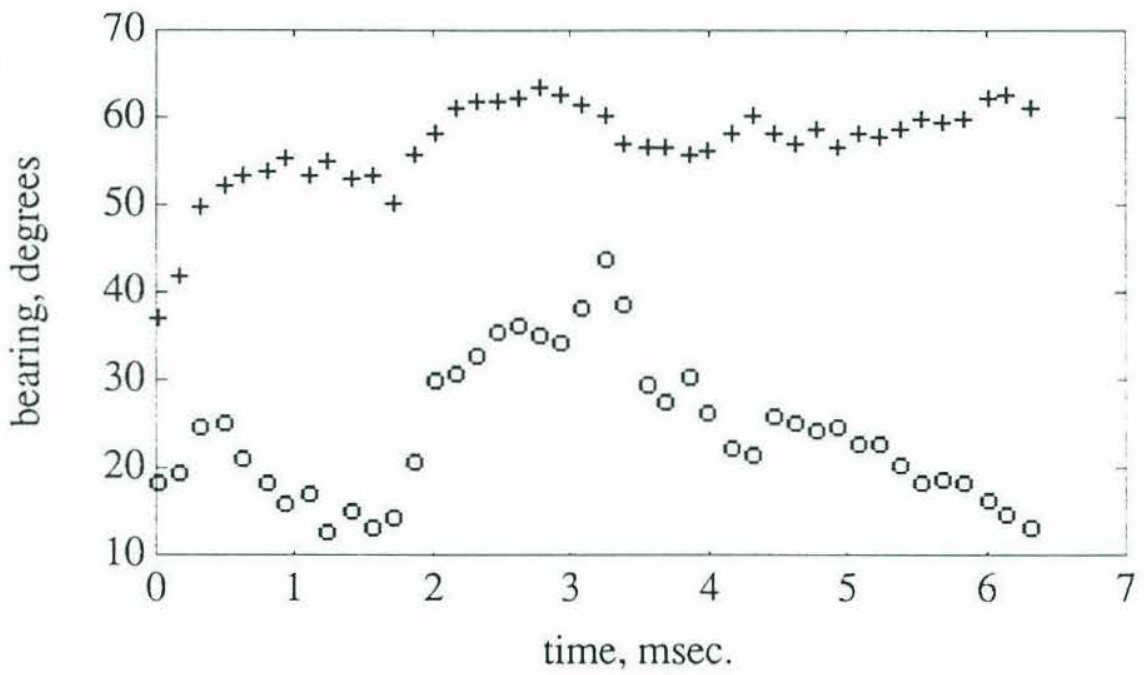

f) $30^{\circ}$; signal power scaled by 50 


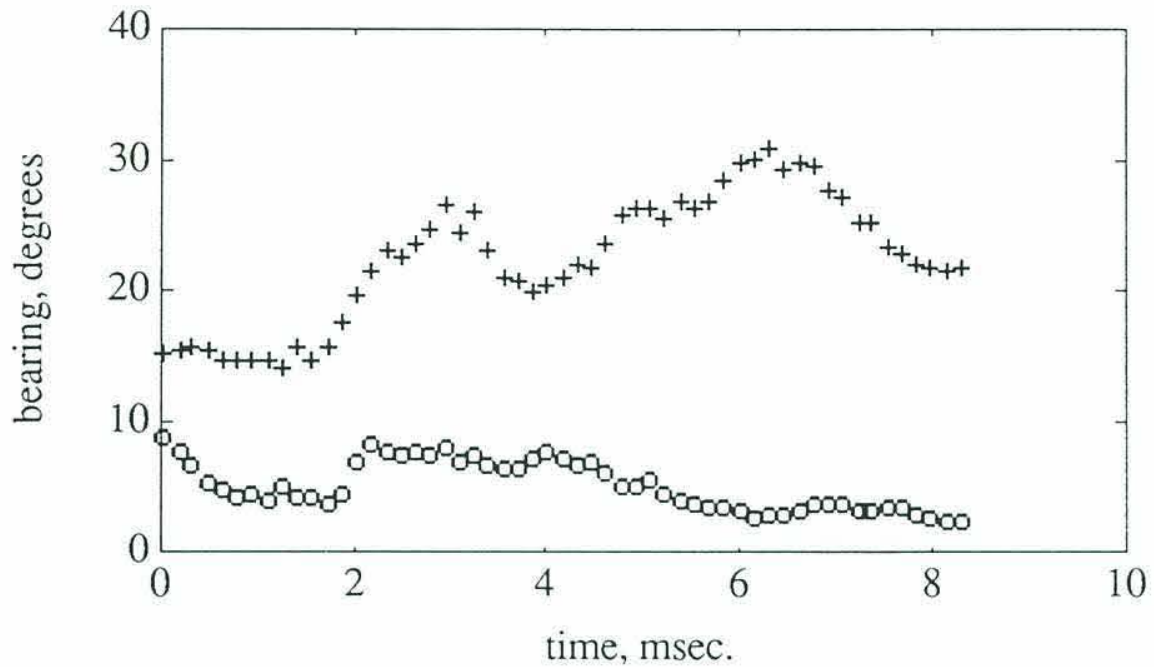

g) $20^{\circ}$; signal power scaled by 100

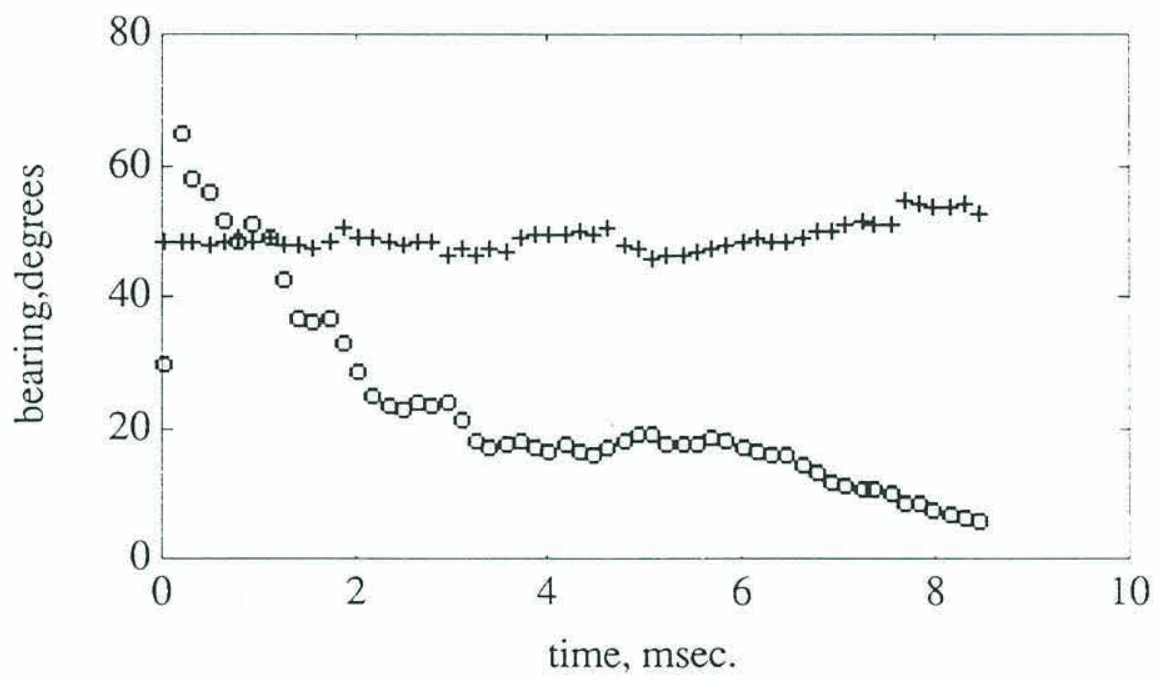

h) $45^{\circ} \# 1$; signal power scaled by 20 


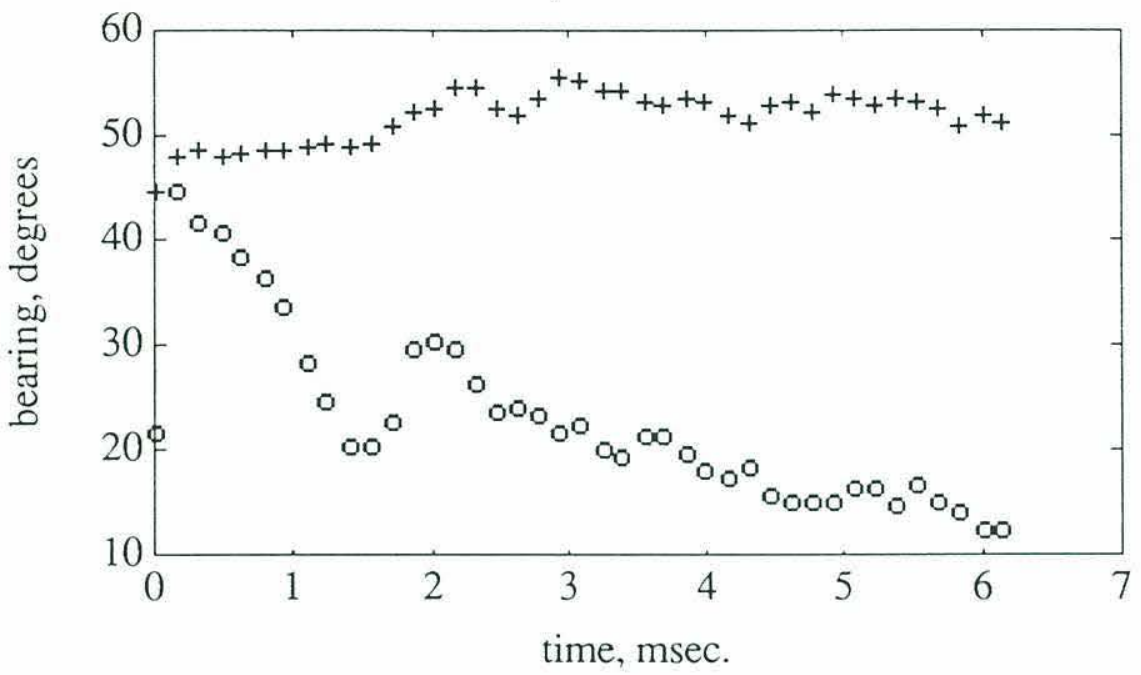

i) $45^{\circ} \# 2$; signal power scaled by 10

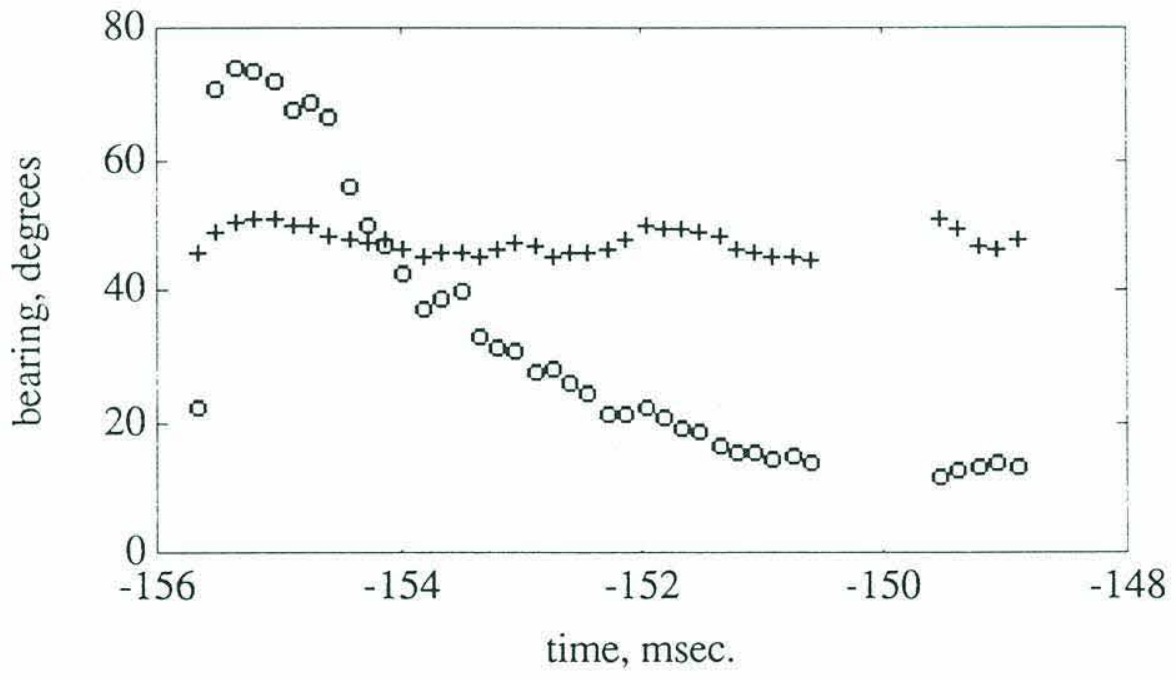

j) $45^{\circ} \# 3$; signal power scaled by 10 


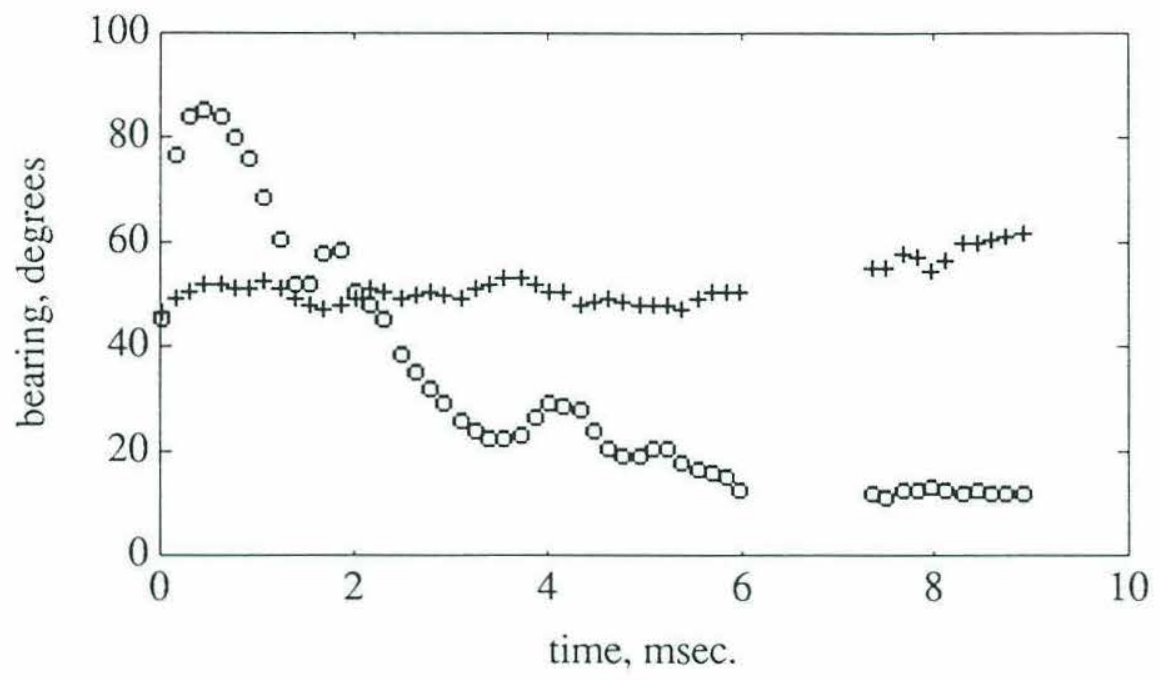

k) $45^{\circ}$ \#4; signal power scaled by 10

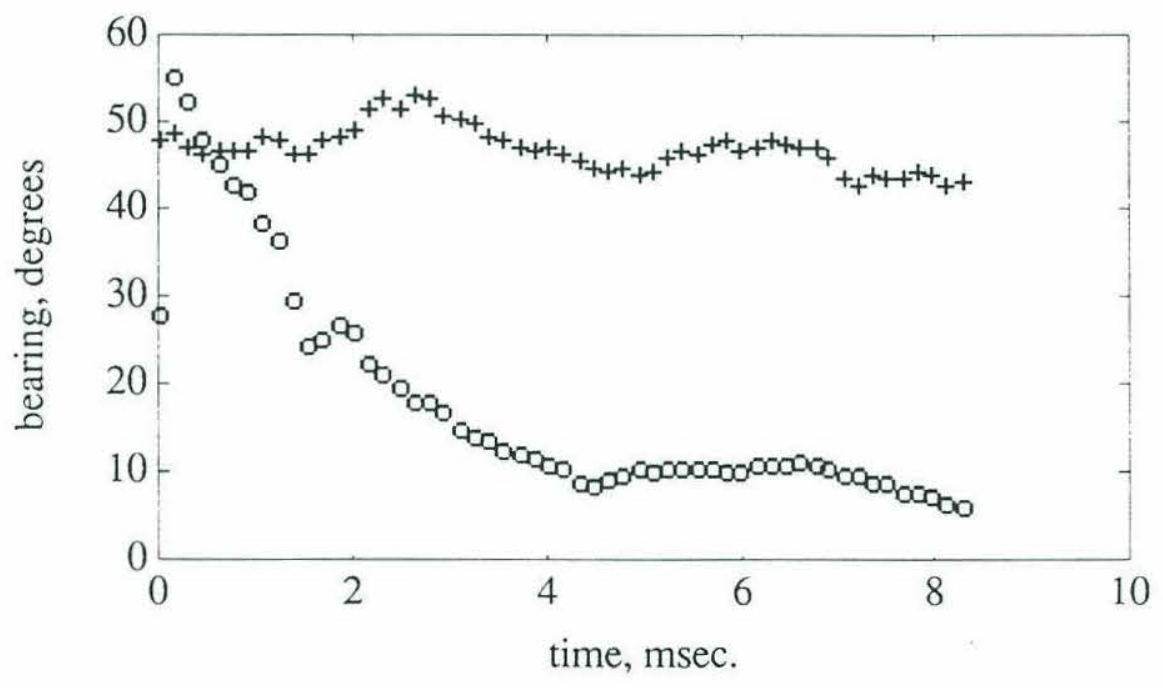

1) $45^{\circ} \# 5$;signal power scaled by 20 


\section{Chapter 6: Conclusions and Recommendations}

\section{$\underline{6.0 \text { Introduction }}$}

In this chapter, the test results presented above are reviewed and conclusions are drawn about system performance. A number of suggestions are then made for improving the system. These suggestions are broken down into hardware and algorithm improvements.

\subsection{Prototype System Performance}

The short-range test results serve to verify that the USBL system can determine bearing with errors of less than a degree at small bearing angles, even with the suboptimal hydrophone mounting used. The short-range tests were also interesting in that they were the only tests for which there was an appreciable amount of time between the direct and surface-reflected arrivals, allowing the averaging of bearing estimates from many times within each pulse. The data logged during these tests can thus be used in future work to develop data filtering schemes for recognizing multipath arrivals.

The longer-range tests show that in shallow water the ability to estimate bearing is limited by interference from multiple arrivals. Interpretation of the test results relies heavily on an understanding of the multipath structure. Tests carried out along the barge show the need for a more accurate hydrophone mounting arrangement. The repeated measurements made to study multipath stability and the effects of pinger orientation show that, even with the effects of pinger ringing transients included, bearing could be measured to an accuracy of $0.7^{\circ}$. Consistent bearing estimation over a wide range of bearing angles was demonstrated.

Further tests need to be carried out at long (>100 meter) ranges to characterize performance at these distances more accurately. The data presented above establish that the 
system can operate over these distances, and indicate that the system can measure bearing with an accuracy of about $1.5^{\circ}$ at small bearing angles.

\subsection{Recommendation for Hardware Improvements}

The tests carried out make it clear that a number of improvements could be made in the test apparatus. Changes could be made in the test setup to make the data easier to interpret. The hydrophones and sound source should be mounted on steel poles, rather than PVC pipes, to minimize motions due to surface waves. A low-Q sound source which could be driven with variable-length tone bursts would be helpful in identifiying multipath arrivals. The sound amplitude could then be controlled, allowing in-water characterization of performance at various signal-to-noise ratios. An effort to determine the shape and depth of the Navy Yard barge hull could be valuable if further testing will continue there.

There are many changes which could be made to the prototype system itself to improve performance. The addition of a second hydrophone baseline would remove much ambiguity in the estimated bearing. With a single baseline, rays incident from any direction along a cone of constant phase difference will yield the same bearing estimate, as discussed in Chapter 2. For the tests presented in this chapter this uncertainty was resolved by placing the source and receiver in the same plane when possible, or by using knowledge of the test geometry. In general operation this will not be possible. This problem can be eliminated by adding a third hydrophone channel and building an L-shaped array.

Pulse detection can also be improved. Currently the information for estimating signal-to-noise ratio is calculated, but not used. The on-line SNR estimate could be used as the detection statistic, as previously discussed. This would result in a detection theshold which does not depend on the front-end gain used, and will reduce the possibility of triggering caused by a broadband noise spike. Perhaps more importantly, the performance model developed in Chapter 3 can be used to predict the expected bearing angle for the 
estimate. The system can then directly output the expected error in each measurement, along with the measurement.

More attention should be paid to the mounting used for the hydrophones. Ideally hydrophone elements would be carefully spaced, sealed in an acoustically transparent epoxy, and calibrated in a test tank. If higher frequencies are to be used, consideration should be given to finding smaller sensor elements.

Finally, much work needs to be done in making the system more user-friendly. Time limitations were encountered in testing due to the need to interrupt the DSP program each time a pulse was observed on the oscilloscope, upload the data, and restart the program. The data then had to be processed by several programs before it could be viewed, meaning that most data was taken "blind" and was not viewed until after the experiment was over. While this arrangement worked well for initial testing, it limited the amount of data that could easily be taken and would be impractical for a tracking system. The DSP board could be interfaced to a portable computer, which could be used to view data and change processing parameters.

\subsection{Recommendations for Algorithm Improvements}

The tests of the prototype system pointed out the importance of being able to recognize the onset of multipath interference. When the data is processed by a human, armed with measurements of the test setup and perhaps a ray-trace program, this recognition problem is not particularly difficult. When the system is implemented on an underwater vehicle, however, the vehicle computer must determine what data is valid. A common solution to this problem, as discussed above, is to chose some preset segment of the pulse to use for bearing estimation, based on assumptions about the expected arrival delays between the different paths. A more satisfying solution would be to implement a flexible algorithm for recognizing the onset of interference on the system's microprocessor. One possible approach would be to statistically filter the bearing estimates. The assumption 
could be made that the first few windows of received data contain information from an uncontaminated direct arrival, and subsequent bearing estimates would be accepted as long as they agreed within some margin of error with the initial estimates.

It seems likely that sizeable performance gains could be made if accurate models of the acoustic propagation were developed. The variability of test results seen in this thesis suggests that any description would need to be statistical in nature. Some form of matched filter processing could then be used to incorporate knowledge of the acoustic environment into the processing. In deeper water tests, multipath arrivals would likely be received which would not overlap each other. In this case, it should be possible to extract useful bearing estimation from several paths, not just the direct path. The usefulness of other arrivals can be determined from further experiments and from modelling the specific ray paths. If an accurate enough propagation model could be developed, it might even be possible to use interpret phase data received during parts of the pulse for which multipath interference is present.

In conclusion, the in-water tests demonstated that the prototype USBL system constructed for this thesis represents a feasible approach to the underwater navigation problem. The system is configured to allow researchers flexibility in modifying the software to fit future needs. Further development could turn the prototype system into a practical navigation system for underwater vehicles. 


\section{Appendix A: Hardware and Electronics}

This appendix discusses the hardware and electronics design done for the prototype USBL system. The hardware is examined in three sections. First, the front-end analog electronics are discussed, then the digital electronics and DSP coding. Lastly, the test apparatus for in-lab performance tests vs. SNR is discussed.

\section{A.1 Front-end Analog Circuitry}

The front-end circuitry was discussed briefly in Chapter 4 . The block diagram of the front-end shown there is repeated below in Fig. A.1. Each component is discussed in detail below.

\section{$\underline{\text { Sound Source }}$}

The sound source used for the experiments was a Datasonics Acoustic Location Pinger ALP 363 . This pinger was nominally tuned to $26 \mathrm{kHz}$, but field measurement showed the transmit frequency to be $25.7 \mathrm{kHz}$. Technical data are shown in Table A.1. Unfortunately a beam pattern was difficult to obtain from the manufacturer.

Table A.1: Datasonics ALP-363 Pinger Specifications

\begin{tabular}{l|l} 
Transmit Power & 20 Watts \\
Source Level & $180 \mathrm{~dB}$ re $1 \mu \mathrm{Pa} @ 1 \mathrm{~m}$. \\
Transmit Pulse Length & $9.6 \mathrm{msec}$. \\
Transmit Pulse Repetition Rate & $5 \mathrm{sec}$. \\
Q (for transducer element) & 5 \\
Operating Depth & $2000 \mathrm{~m}$. \\
Size & $4^{\prime \prime} \times 10^{\prime \prime}$ \\
\hline
\end{tabular}




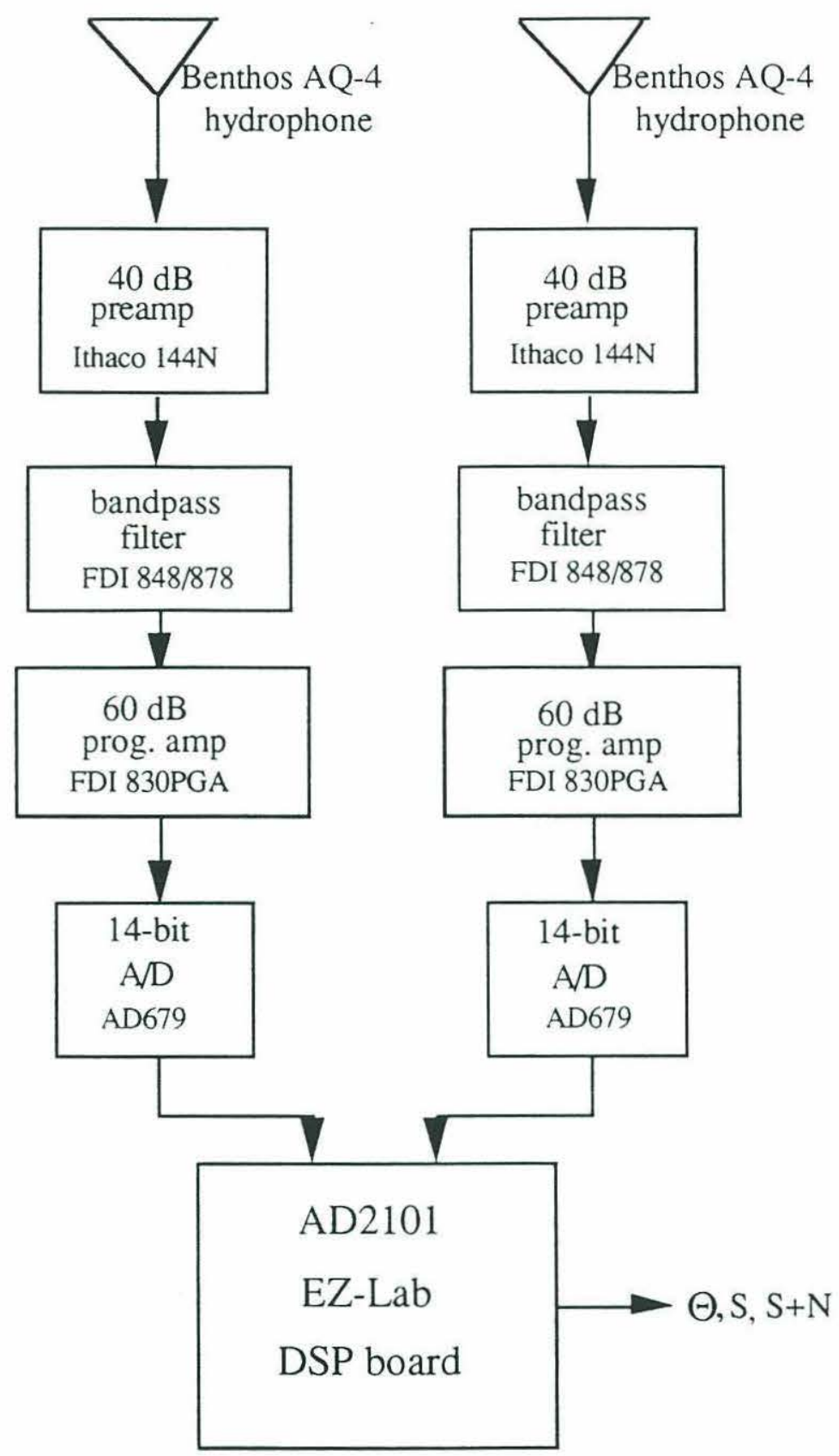

Figure 1: Front-End Electronics Configuration 
Table A.2: Benthos AQ-4 Hydrophone Specifications

\begin{tabular}{|l|l} 
Sensitivity & $-201 \mathrm{~dB}$ re $1 \mathrm{~V} / \mathrm{mPa}$ \\
Frequency Response & $1 \mathrm{~Hz}-35 \mathrm{kHz}$ \\
Size & $1.3 \times 3.3 \mathrm{~cm}$. \\
Depth Rating & $3300 \mathrm{~m}$. \\
Capacitance & $2400 \mathrm{pF}$ \\
\hline
\end{tabular}

Table A.3: Ithaco 144N Preamplifier Specifications

\begin{tabular}{|l|l|}
\hline Gain & $40+-0.2 \mathrm{~dB}$ \\
Frequency Response & $0.65 \mathrm{~Hz}$ to $200 \mathrm{kHz}$ \\
\multicolumn{1}{|c|}{$100 \mathrm{pF}$ Source } & $2.4 \mathrm{mVrms}$ \\
Output Impedance & $8 \mathrm{mVrms}$ \\
Supply Voltage & 35 ohms in series with $40 \mathrm{mF}$ \\
Supply Current & $+15 \mathrm{~V} \cdot$ to $+30 \mathrm{~V}$. \\
Maximum Output, & $10 \mathrm{~mA}$ to $20 \mathrm{~mA}$ \\
Maximum Output, current & $9 \mathrm{~V}$ to $18 \mathrm{~V}$ \\
DC Voltage at Ouput & $4 \mathrm{~mA}$ to $10 \mathrm{~mA}$ \\
Power Supply Isolation & $0 \mathrm{~V}$ \\
Weight & $65 \mathrm{~dB}$ \\
\hline
\end{tabular}




\section{Hydrophones}

The hydrophones used were Benthos AQ-4 's, donated to Sea Grant by the manufacturer. Technical specifications for these hydrophones are seen in Table A.2. Ideally the sensors for a USBL system would be smaller and more sensitive, but these requirements are often mutually exclusive. The hydrophone capacitance must be compared to the cable capacitance, as the capacitances will lead to an attenuation of the signal level; for the prototype USBL setup with 30 ' of cable the attenuation will be about $30 \%$, which is acceptable.

\section{Preamplifiers}

Ithaco $144 \mathrm{~N}$ preamplifiers were chosen because of their small size and relatively low noise. Specifications are shown in Table A.3. The preamplifiers were modified by Ithaco to add a highpass $\mathrm{RC}$ filter with a $5 \mathrm{kHz}$ cutoff frequency. This was done in order to avoid swamping the preamplifiers with low frequency shipping noise. The preamplifiers are physically small and have low current drain, so they could be used as part of a compact vehicle-based implementation of this system.

\section{Filters}

Programmable 8-pole highpass and lowpass filters built by Frequency Devices, Inc. were chosen for filtering the received signal. Bandpass filtering is done to prevent saturation in the amplifier stage of the electronics. The programmability of the filters increases the size of the unit, but adds needed flexibility for system development and dockside testing. Simple dip switch circuits were built to allow easy selection of cutoff frequency. Technical specifications for the filters are seen in Figs. A.2, A.3, and A.4. The phase match between channels is $0.5^{\circ}$; this is helpful, as the reasonably close matching allowed initial tests to be carried out without calibration of the relative phase shifts introduced into the different channels. 


\begin{tabular}{ll}
\hline -3dB Corner Frequency & $\pm 2 \%$ \\
\hline Passband Ripple (theoretical) & \\
Butterworth & $0.0 \mathrm{~dB}$ \\
Bessel & $0.0 \mathrm{~dB}$ \\
Linear Phase & $0.015 \mathrm{~dB}(60 \mathrm{~dB}$ model) \\
& $0.15 \mathrm{~dB}(80 \mathrm{~dB}$ model $)$ \\
Elliptic & $0.035 \mathrm{~dB}$
\end{tabular}

Typical Noise (Butterworth, Bessel, Linear Phase)

Narrow Band $\quad$ ( $5 \mathrm{~Hz}$ to $50 \mathrm{kHz}$ ) $50 \mu \mathrm{V}$ rms

Wide Band $\quad(5 \mathrm{~Hz}$ to $2 \mathrm{MHz}) 200 \mu \mathrm{V}_{\mathrm{ms}}$

Typical Noise (Elliptic)

Narrow Band $\quad(5 \mathrm{~Hz}$ to $50 \mathrm{kHz}) 75 \mathrm{uV}$ rms

Wide Band $\quad$ (5Hz to $2 \mathrm{MHz}$ ) $250 \mu \mathrm{V}_{\mathrm{ms}}$

Phase Match (All models) ${ }^{2}$

@ fc ( $\mathrm{fr}$ Typical $\pm 1.0^{\circ}$. Maximum $\pm 2.0^{\circ}$

Passband Typical $\pm 0.5^{\circ}$. Maximum $\pm 1.0^{\circ}$

Gain Match (All models) ${ }^{2}$

@ic (fr)

Typical $\pm 0.1 \mathrm{~dB}$. Maximum $\pm 0.2 \mathrm{~dB}$

Passband

Typical $\pm 0.05 \mathrm{~dB}$. Maximum $\pm 0 . i \mathrm{~dB}$

Distortion' ( For range $-1,-2,-3,-4$, all lowpass models)

\begin{tabular}{lll} 
Typical & $0.002 \%$ & $(-94 \mathrm{~dB})$ \\
Maximum & $0.004 \%$ & $(-88 \mathrm{~dB})$ \\
\hline
\end{tabular}

Distortion' (For range -5 - all lowpass models)

Typical $\quad 0.004 \%(-88 \mathrm{~dB})$

Maximum $\quad 0.025 \%(-72 \mathrm{~dB})$

Note

1. For input signais $\leq 3 \mathrm{~V}_{\mathrm{rms}}$ the total harmonic distortion is $\leq 0.01 \%$ $(-80 \mathrm{~dB})$ for all five ranges.

2. Unit to unit match for the same transfer function set to the same frequency.

Figure 2: Filter Module Specifications 


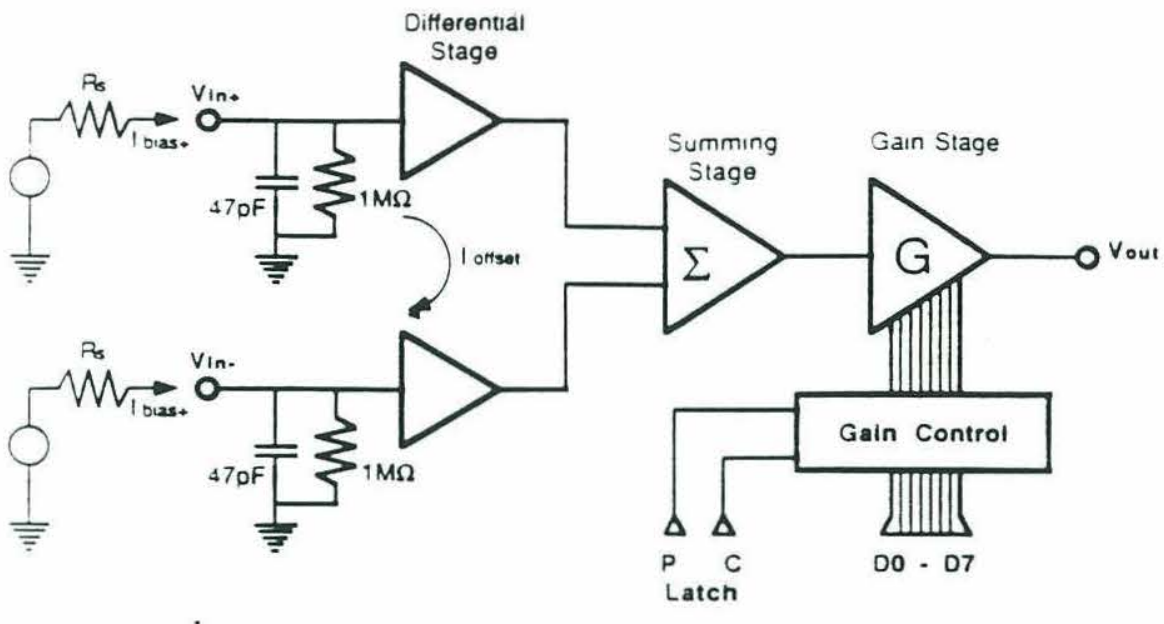

\section{General Analog Characteristics}

\begin{tabular}{ll}
\hline Gain & $0 \mathrm{~dB}$ to $60 \mathrm{~dB}$ in $2 \mathrm{~dB}$ Steps \\
& $0 \mathrm{~dB}$ to $40 \mathrm{~dB}$ in $1 \mathrm{~dB}$ Steps \\
& $0 \mathrm{~dB}$ to $20 \mathrm{~dB}$ in $0.5 \mathrm{~dB}$ Steps \\
Gain Tolerance & $\pm 0.02 \mathrm{~dB}$ \\
Gain Match (unit to unit @ $0 \mathrm{~dB}$ ) & $0.04 \mathrm{~dB}$ from $1 \mathrm{~Hz}$ to $100 \mathrm{kHz}$ \\
Gain vs. Temp. @ G = $0 \mathrm{~dB}$ & $0.001 \mathrm{~dB} /{ }^{\circ} \mathrm{C}$ \\
& $\left(0.01 \% /^{\circ} \mathrm{C}\right)$ \\
Gain vs. Temp. @ G = $60 \mathrm{~dB}$ & $0.005 \mathrm{~dB} /{ }^{\circ} \mathrm{C}$ \\
& $\left(0.05 \% /{ }^{\circ} \mathrm{C}\right)$ \\
Phase Match (unit to unt @ $0 \mathrm{~dB})$ & $0.5^{\circ} \mathrm{from} 1 \mathrm{~Hz}$ to $100 \mathrm{kHz}$ \\
Distortion & $0.003 \% @ 1 \mathrm{kHz}$ \\
& $0.02 \% @ 90 \mathrm{kHz}$ \\
Full Power Bandwidth & $100 \mathrm{kHz} \mathrm{min}$.
\end{tabular}

Figure 5: Programmable Filter Specifications

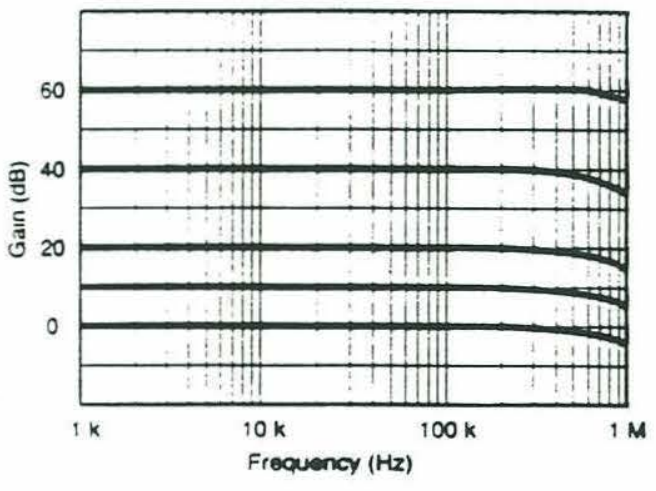

Frequency Response

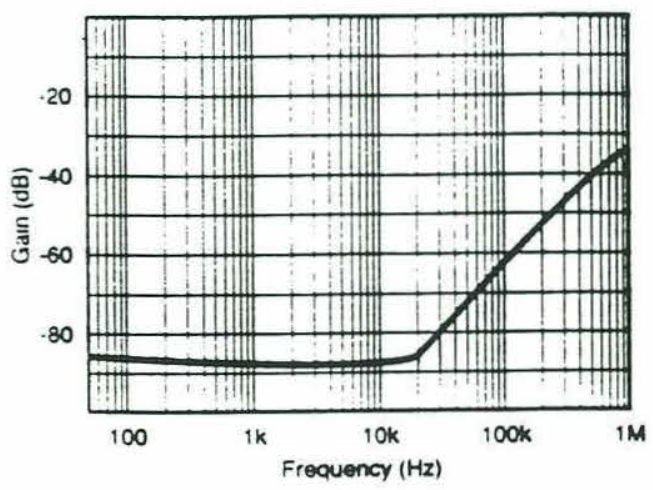

Common Mode Rejection Ratio (CMRR) 
The front-end electronics were selected to be compatible with tests of a wideband USBL system, although these were never carried out. For this implementation, it is important to have no dispersion introduced by the front-end system. For this reason $80 \mathrm{~dB}$ linear phase filters were chosen for the lowpass filters; Fig. A. 4 shows that the group delay is nearly constant for frequencies below the cutoff frequency. Butterworth filters were chosen for highpass filters (see Fig. A.3). These filters have a faster amplitude rolloff which leads to more complete filtering of low frequency shipping noise, but introduce uneven group delays for frequencies near cutoff. It can be seen that in order to avoid dispersion, the cutoff frequency for the highpass filters would have to be set at about half the lowest frequency of interest.

\section{$\underline{\text { Amplifiers }}$}

Programmable amplifiers from Frequency Devices, Inc. were used after filtering to boost the signal further. These amplifiers provide up to $60 \mathrm{~dB}$ of gain, as seen in Fig. A.5. Simple circuits were constructed to use a dip switch to select gain, although the gain could be controlled by the processor for an on-vehicle implementation. Combined with the preamplifiers, a total gain of $100 \mathrm{~dB}$ was possible.

\section{Front-end Construction: Grounding, Shielding, and Noise}

The amplifier and filter units chosen for the front-end analog circuitry have been described above. In order to reach noise and performance levels close to those specified by the manufacturer, careful attention was paid to circuit construction. The important issues are outlined below, along with the solutions arrived at for this system.

\section{Grounding Problems}

The most common type of grounding problem is the infamous ground loop. This occurs whenever two points in the circuit are grounded so that there is a potential difference between them, perhaps due to resistance in a connecting wire. This offset is seen by the amplifiers and amplified, leading to contamination of the signal. Ground loops are most 
effectively avoided by providing a separate return to the power supply ground at every grounded point in the circuit. (Meiksin and Thackray, p. 370-371).

\section{Shielding and Interference}

Electromagnetic noise present near the electronics will be picked up and amplified by the system. In order to minimize its effect, the noise sources and coupling mechanisms must first be clearly understood. Simple rules for shielding the electronics can then be applied.

The main sources of noise at the Charlestown navy yard include AM and marine radio transmissions. These noise sources can capacitively couple into the signal lines and electronics. It should be noted that the $\mathrm{AC}$ power line going to the Sea Grant Charlestown shack is quite noisy, and devices running off line current will have problems with noise entering through the power supply. For this reason it was decided to run all front-end and DSP circuitry off battery power.

The capacitatively coupled noise sources can be controlled by using proper shielding. Shields are effective because electric charge is restricted to the surface of conductors. The electronics should therefore be enclosed by a closed metal shell and the noise voltages created on the shell should be shunted to ground. Alan Rich ("Shielding and Guarding" p.124-125) gives a simple set of rules for effective shielding. These are summarized briefly here:

1) To be effective, the shield must be connected to the reference potential of the signal. Ideally, the connection should be as close to the signal source as possible. For the USBL application this means the connection should be at the hydrophone itself, which presents some construction problems. The solution below is a compromise, with the connection at the preamp.

2) If the shield is broken up (i.e. cable shield-instrument case-shield-case) the shields must be tied together, and have only one connection to ground. 
3) Each signal should have a separate shield, connected to its own reference potential.

4) Any current or voltage on the shield with respect to the signal's reference potential should be avoided. Since there is capacitance between the shield and center conductors, any voltage will couple to the signal leads.

5) Careful attention should be paid to the return route from shield to ground. This return should be as short as possible.

Fig A.6 shows the grounding and shielding scheme for the USBL front end. This layout follows the rules Rich sets forth, except for grounding the shield at the preamp and not the hydrophone.

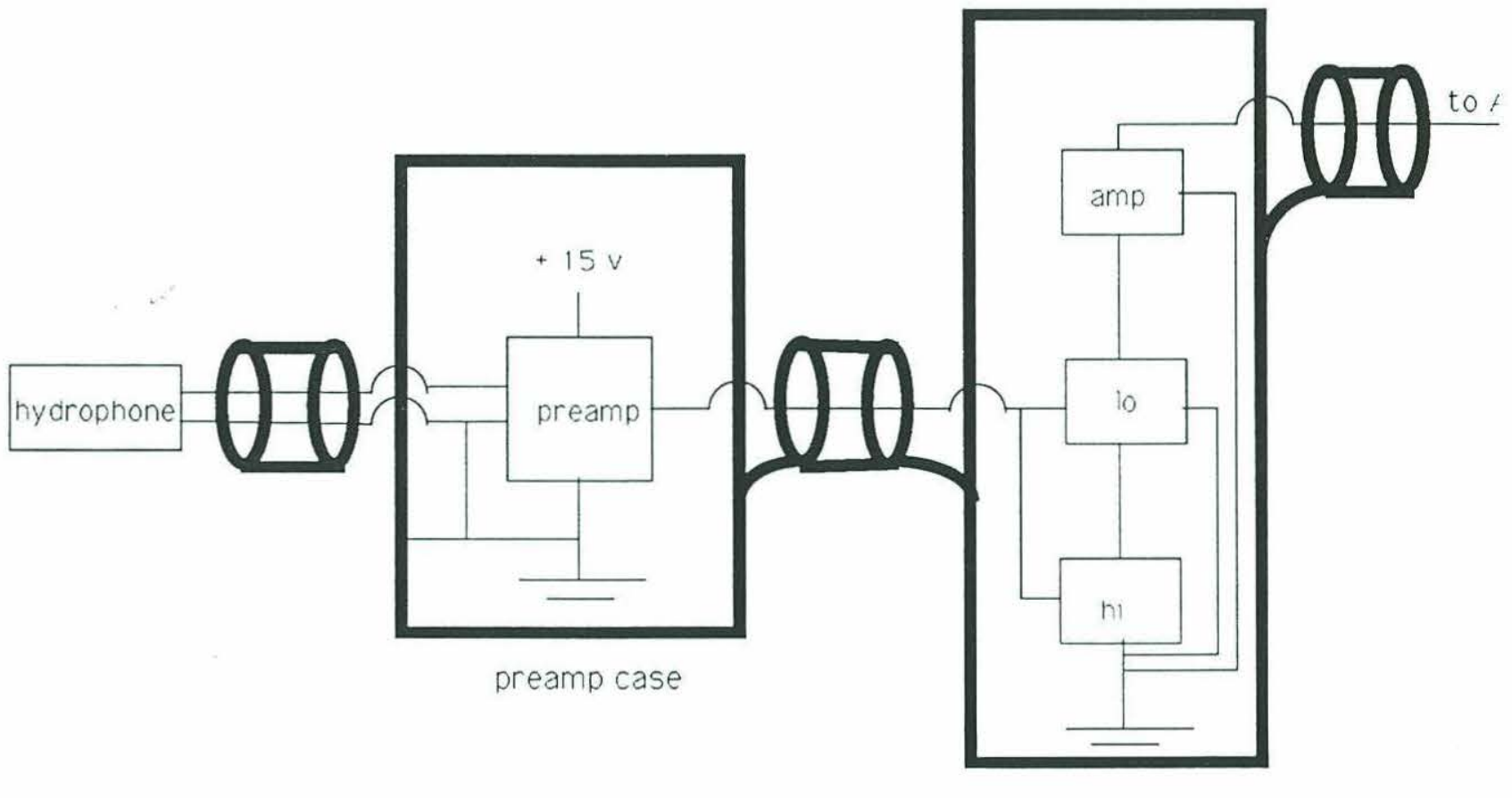

Figure A.6: Grounding and Shielding for Front-end Electronics

Unfortunately, the grounding and shielding layout above will not solve all the problems. At high frequencies the Common-Mode Rejection Ratio (CMRR) of the differential amplifiers falls off, meaning the amplifiers will have trouble rejecting AM radio pickup present on both signal lines. The falloff in CMRR can be compensated for by 
wrapping the cables a few times around a ferrite core (Horowitz and Hill, p.311-312). This technique, known as an RF choke, introduces an impedance in series with the shield and the cables, raising the impedance at high frequencies. A pair of small $(0.01 \mathrm{mF})$ capacitors can be used to shunt high frequency signals to ground.

The noise produced by capacitive coupling to the hydrophone cables can be reduced further by connecting the preamplifier inputs with a resistor. This helps because capacitive coupling produces a noise current in the cables (Rich, "Understanding Interference-Type Noise"). If a resistor value $\mathrm{R}$ which is much lower than the amp's input impedance is used, the noise current will develop most of its voltage drop across the resistor, not across the amplifier. The transducer will drive almost no current but produces a voltage difference, which will be seen at the amplifier regardless of the value of $\mathrm{R}$.

\section{A.2 Digital Electronics and DSP Coding}

The digital electronics for the test setup consisted of a data acquisition card and the DSP board itself. These will be described briefly below, as will the program written for the DSP microprocessor to calculate phase differences.

\section{Data Acquisition Board}

A small data acquisition board was built to digitize received signals and pass the results on to the AD2101 DSP board. Analog Devices AD679 14-bit A/D converters were used. These chips were donated to Sea Grant by Analog Devices. This board was connected to the EZ-Lab DSP board by a ribbon connector to the parallel port, and a few lines out of the EZ-Lab serial port.

The operation of the data acquisition circuit, shown in Fig. A.7, is straightforward. The AD2101 DSP chip supplies a serial clock timing line to the AD679 converters. A falling edge on the SC line triggers a conversion. The completion of conversion is signaled by the end-of-convert (EOC) pin, which acts as a high priority interrupt to the DSP chip via 
the IRQ2 line. The DSP begins to read the data values in by asserting the address of an A/D converter on its external address bus. Two bits of the address are used in a simple address decoding scheme. A $74 \mathrm{HC} 154$ multiplexer chip drops one of its output lines low depending on the address seen on its inputs (A, B, C, and D below). This ouput is OR'ed with the inverted read line from the DSP. When the DSP is attempting to read one of the chips, both lines drop low and the output enable (OE) pin is enabled. The DSP can then read out the first 8 bits of the 14-bit A/D output (the AD679 assumes an 8-bit bus is available). The DSP then asserts the address of the other A/D converter, and follows the same steps to read in its conversion result. The DSP then uses its flag line F0 to toggle the high-byte-enable (HBE) pin of the AD679, and reads out the remaining bits of the conversion for each channel. The bytes are shifted and combined in the DSP code to produce a single 14-bit value. 


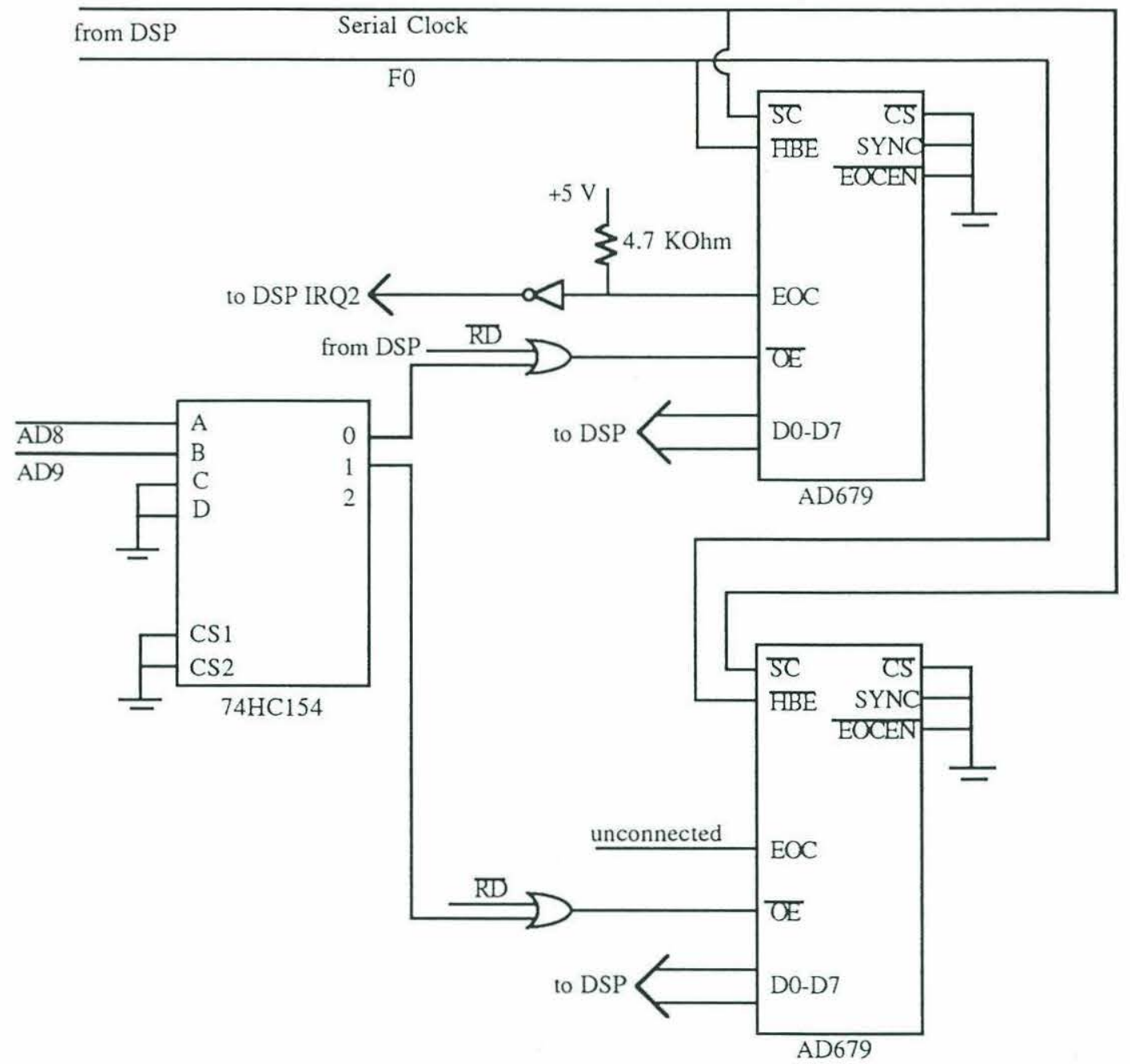

Figure A.7: Diagram for Data Acquisition Circuit 


\section{$\underline{\text { AD2101 DSP Board }}$}

The DSP microprocessor used was an Analog Devices AD2101 fixed-point 12.5 $\mathrm{MHz}$ chip. An Analog Devices product, the EZ-Lab development board, was used as a development base. The EZ-ICE in-circuit emulator was used during testing; firmware on the emulator allowed code developed on any IBM compatible machine to be downloaded to the DSP, and processed data from the DSP could be logged on the IBM. The EZ-Lab has two serial ports and one parallel port available. Both were used for communication and timing for the data acquisition board.

Code for the AD2101 was developed in assembly language. Program developing and debugging were facilitated by the in-circuit emulator. Filter coefficients and sinusoidal waveforms were generated in MATLAB, then converted to the hexadecimal fractional format used by the DSP code. Similarly, data logged from the DSP was converted into MATLAB format and analyzed. Much more information is available about the DSP hardware and development process, but is not required for an understanding of what was done in this thesis. For further information, the reader is referred to the Analog Devices literature.

The flowchart of the main USBL program is seen in Figure A.8. A printout of this program is seen at the end of the Appendix. After initialization, the processor spends most of its time in a low-power wait mode, waiting for interrupts. In the current USBL program, the processor spends $85 \%$ of the time in the wait state. The chip's power consumption is $750 \mathrm{~mW}$ active, versus $80 \mathrm{~mW}$ in wait mode. The highest priority interrupt is received on the IRQ2 line, which tells the processor that the A/D converters have finished conversion and should be read. The data values are then read into two memory buffers, one for each channel. The processor checks to see if these buffers are full, and if so generates a low-level interrupt signaling that the buffers are filled and ready for processing. All A/D interrupt processing operations are carried out using a secondary set of registers, 


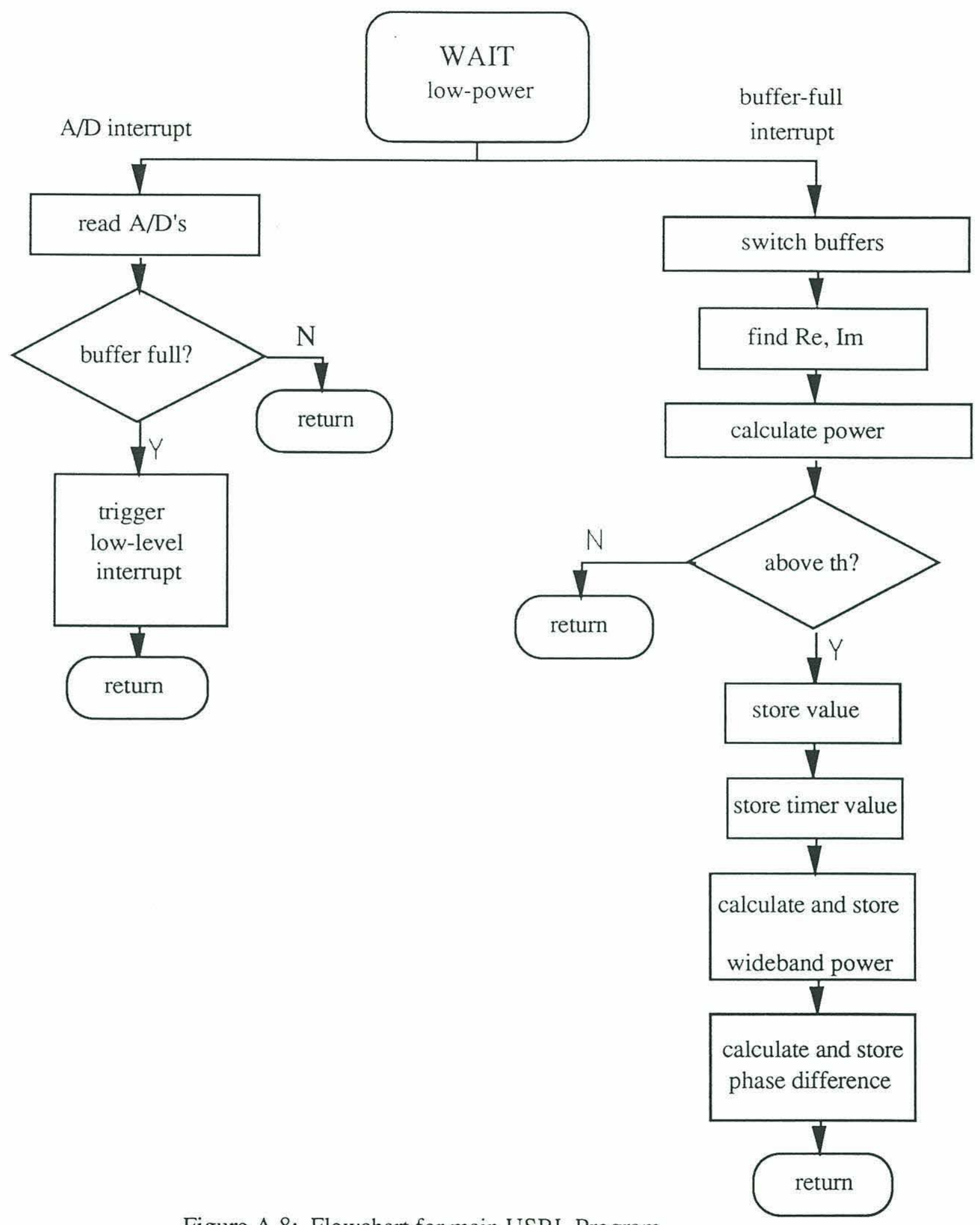

Figure A.8: Flowchart for main USBL Program 
so the data processing carried out in the primary registers will not be disturbed by the periodic interrupts from the $\mathrm{A} / \mathrm{D}$ converters.

The low level interrupt triggers the processing of the received data. Before processing begins, the full buffers are switched with a spare pair of buffers. This is done to avoid overwriting the older data with fresh data while processing is being carried out. Next, the data from each channel is multiplied by a sine and cosine at the broadcast frequency $f_{O}$ and summed to find the real and imaginary parts of the Fourier transform at $f_{0}$. These components are then squared and summed to calculate the signal power, which is compared to a threshold. If the threshold is exceeded, the signal (inband) power is stored in memory. The wideband power and phase difference are calculated and also stored in memory, and the DSP timer value is read to time-stamp the data packet. The processor then returns to waiting for the next interrupt.

\section{A.3 In-Lab Noise Performance Measurements}

Measurements were made in the laboratory of USBL system performance at various signal-to-noise ratios. The results from these measurements were presented in Chapter 2. The circuit used to mix signal and noise voltages is shown in Fig. A.9. The signal generator used was an Intersil ICL8038 precision function generator chip which was used to generate a precisely tunable sinewave output. This output was split and buffered to create two independent signal channels. One of these channels was run through an $\mathrm{RC}$ filter with a knee near the signal frequency, which attenuated the signal somewhat but more importantly introduced a phase shift. The other, unshifted channel was attenuated using a variable resistor as a voltage divider to equalize the amplitudes on the two channels. The two channels were now buffered once more and fed into a summing amplifier along with the noise signals. Figure A.10 shows the schematic for the noise generators used, which were taken from a handbook of electronic circuits [Graf, 1985]. This circuit basically amplified the transition noise from a transistor junction to provide a noise voltage. The 
signal-to-noise level was set by changing the values of the weighting resistors R3 and R4.

Thus the output of the two summing amplifiers gave two signals with a relative phase shift between them, with independent noise added to each channel. The phase difference could be changed by tuning R2 and changing the RC filter cutoff frequency, and SNR was controlled by changing the ratio of R3 to R4. The SNR was measured from peak-to-peak voltages on an oscilloscope.

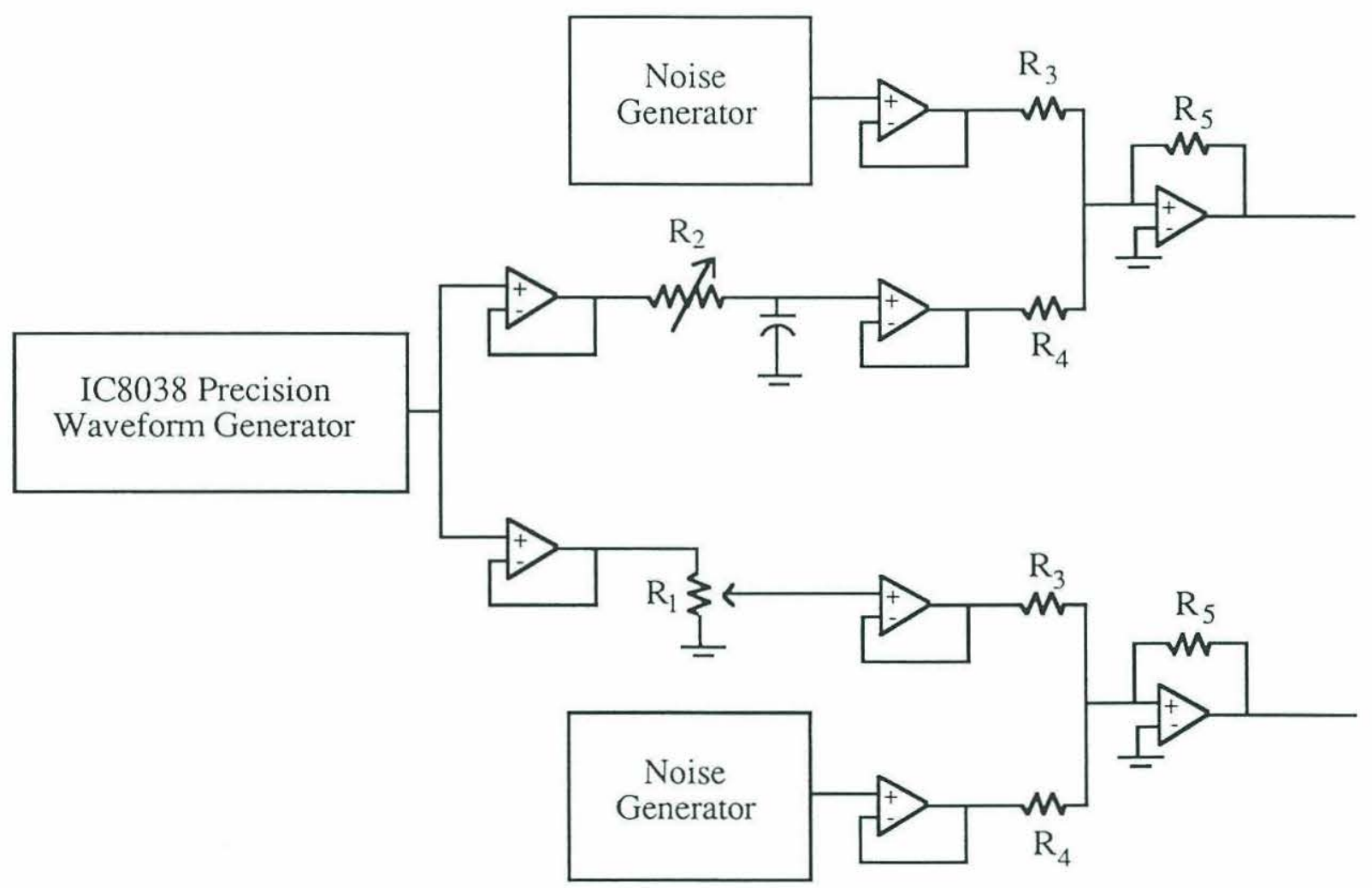

Figure A.9: Signal-to-Noise Test Input Generator 


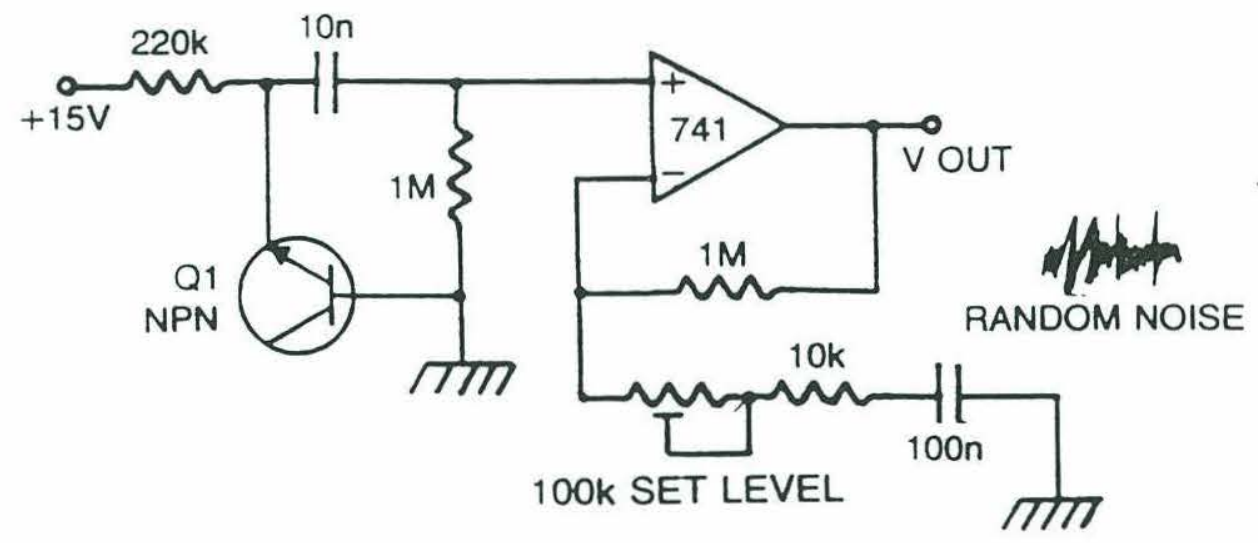

Figure A.10: Noise Generator Circuit 


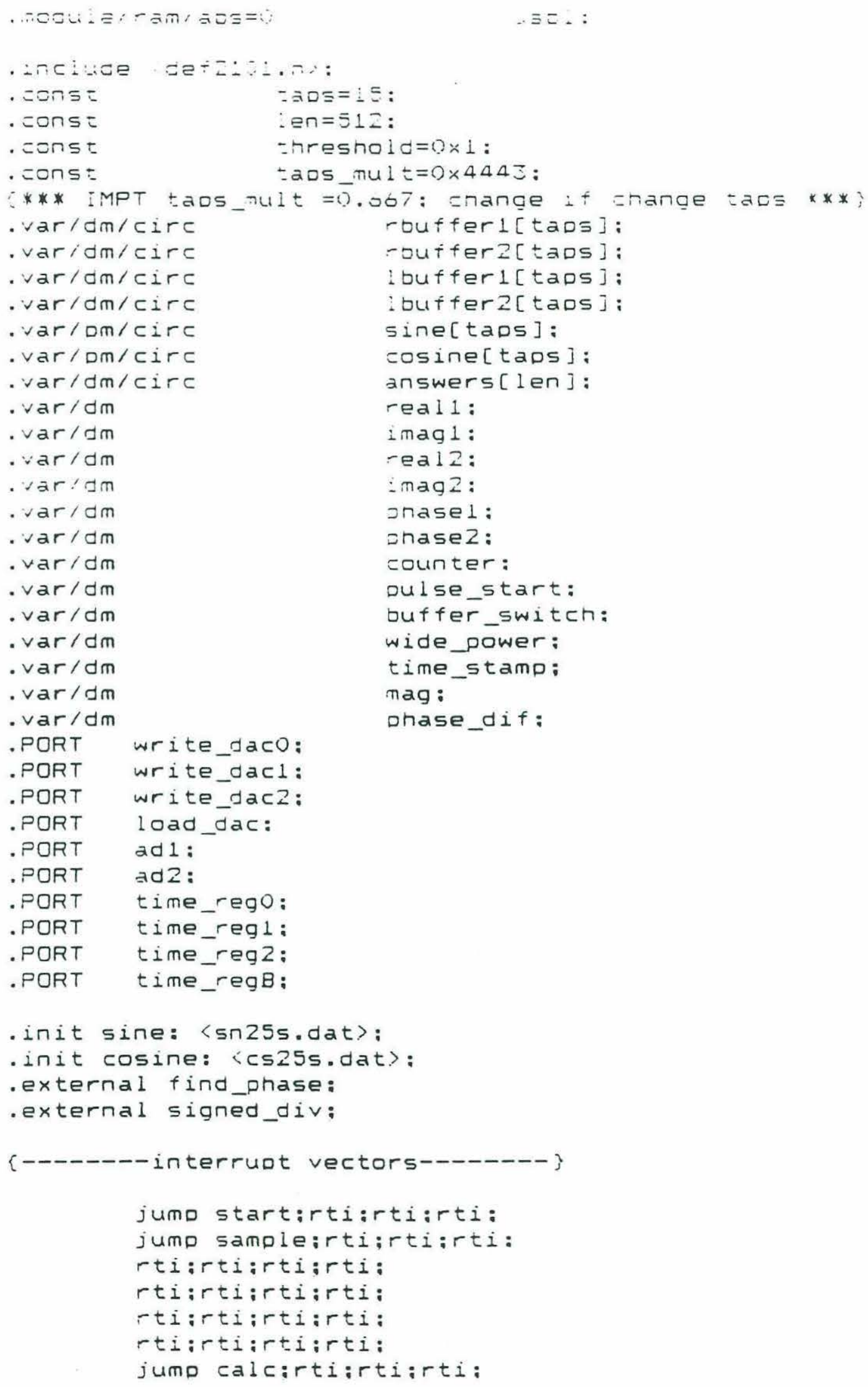




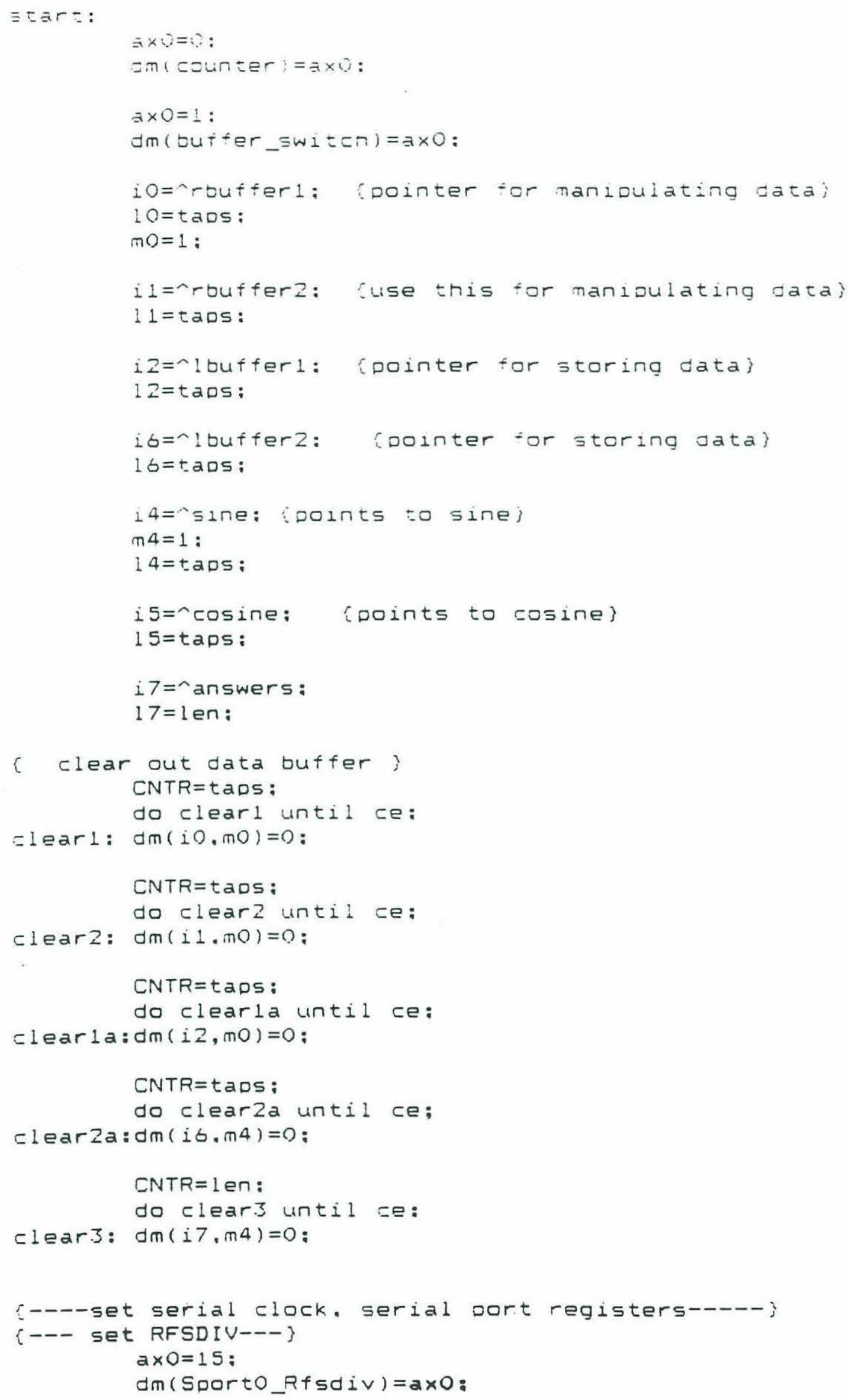




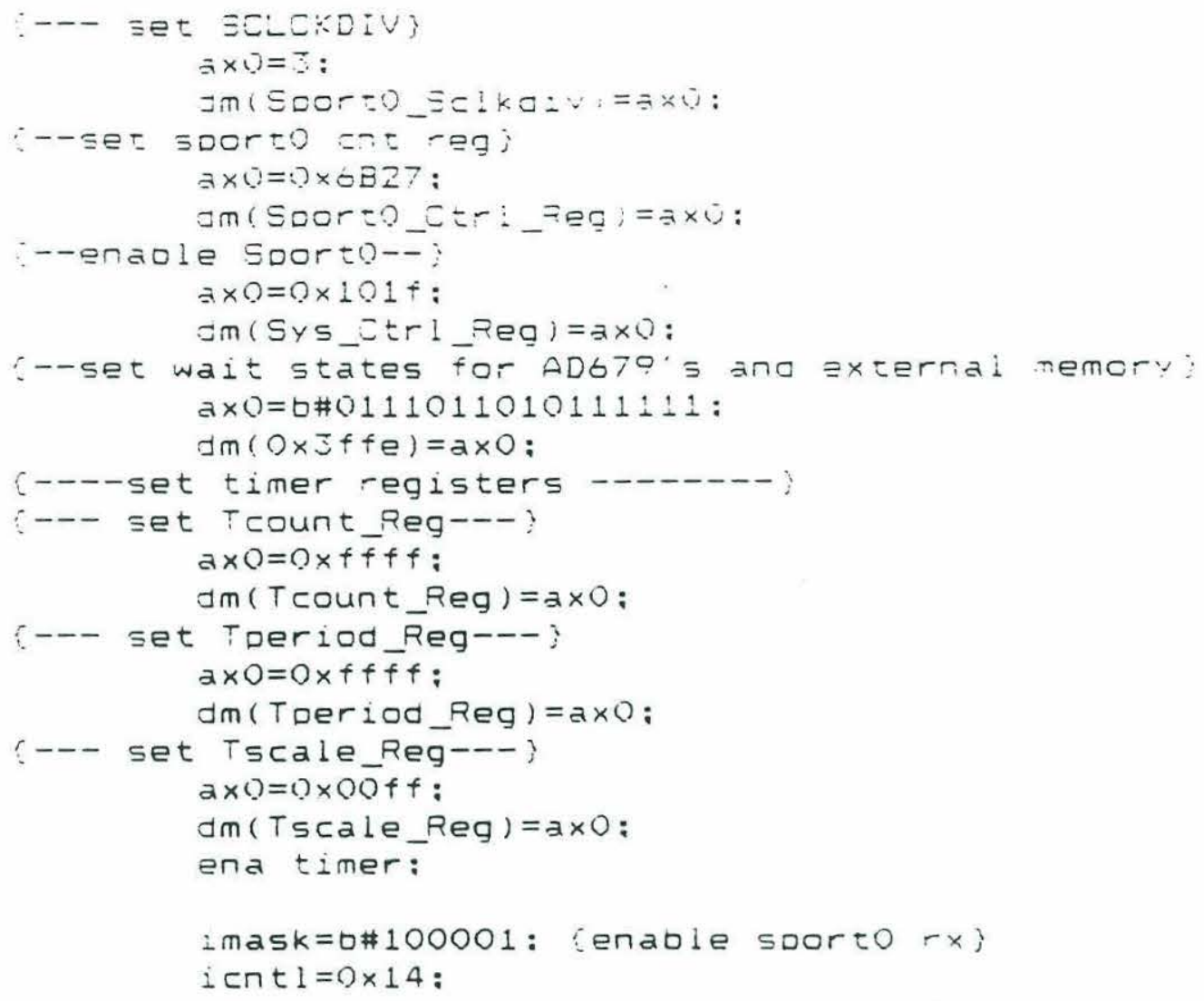




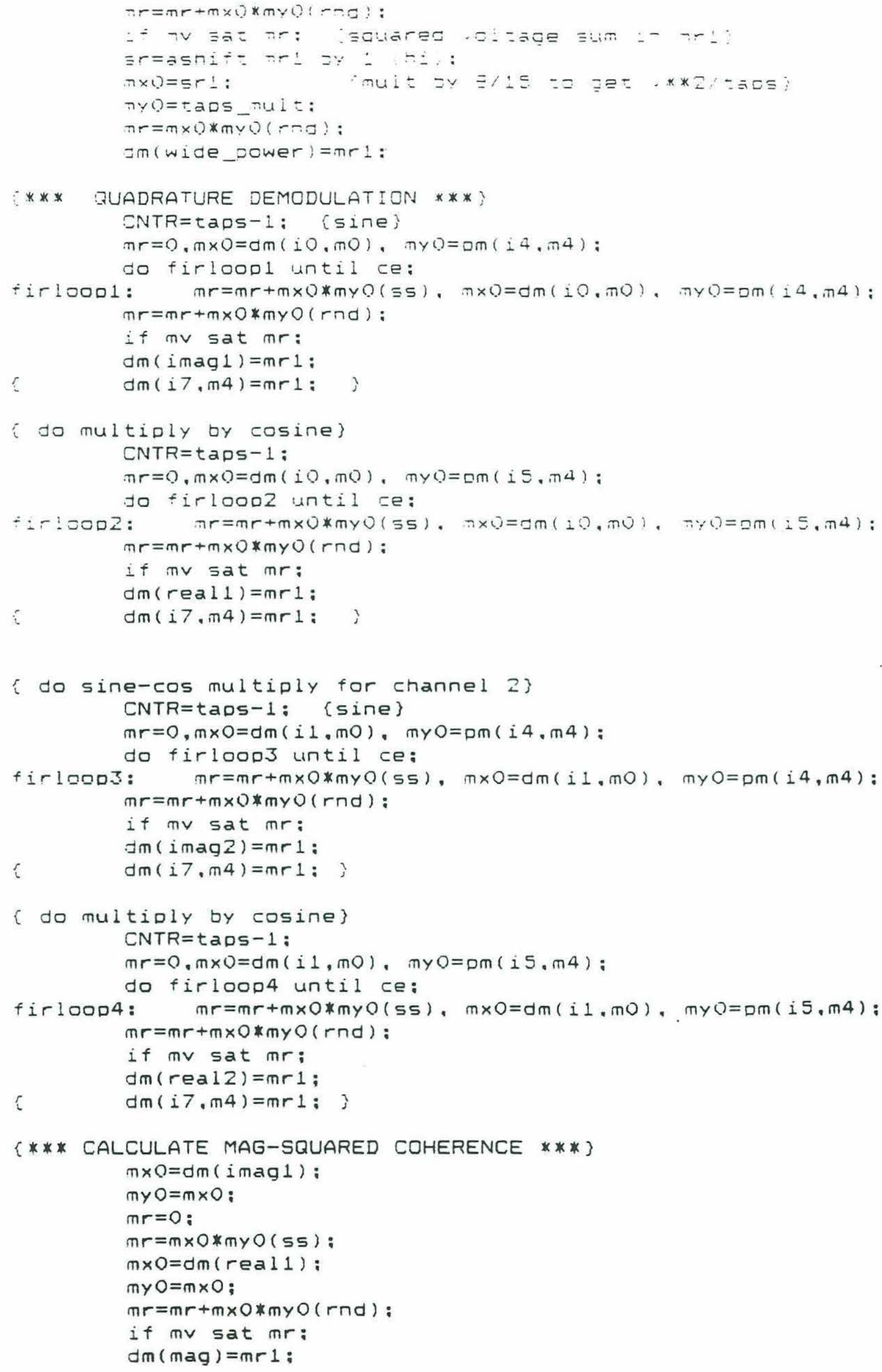




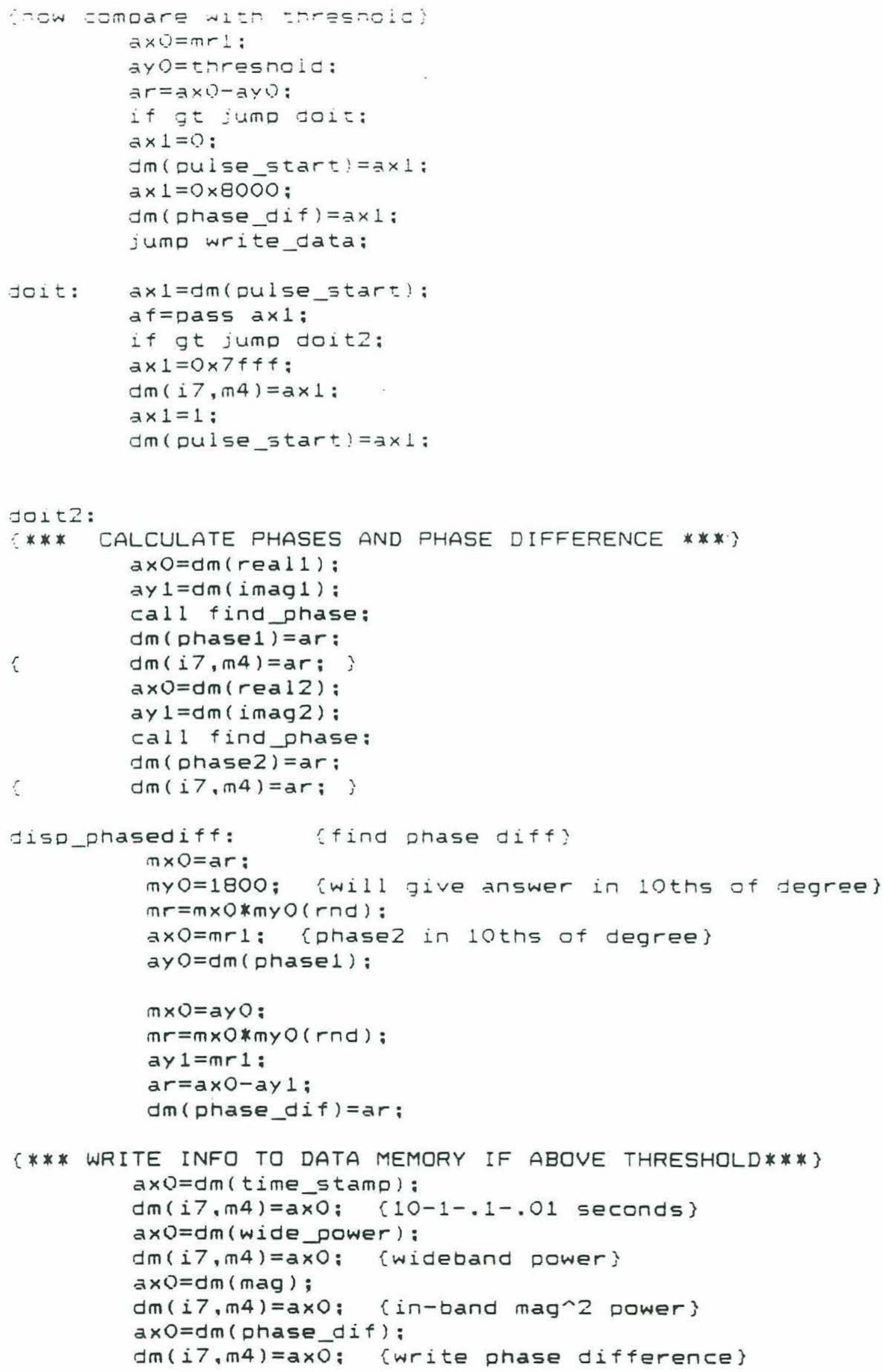




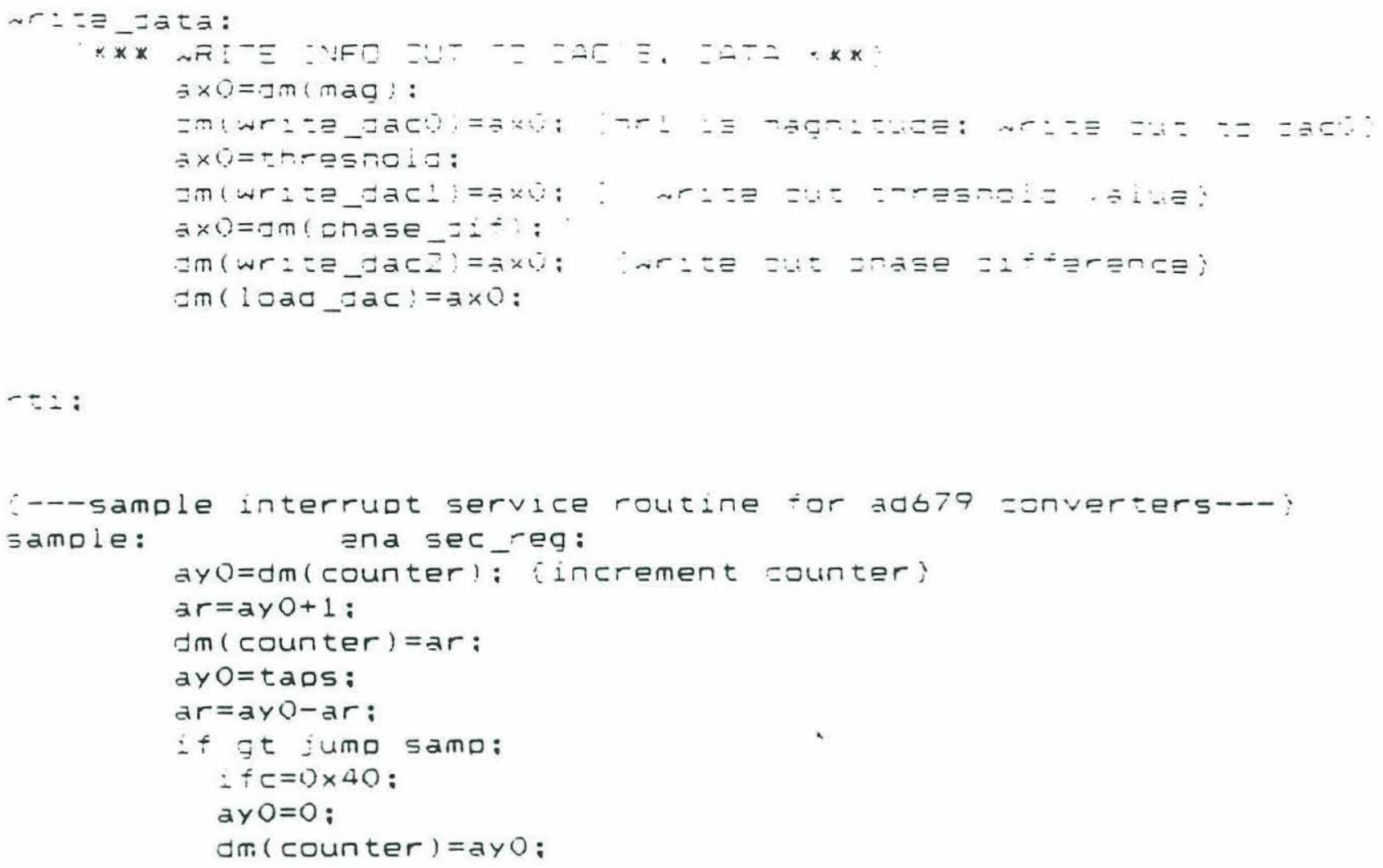

इamp:

reset flag_out:

nop;

$\operatorname{axO}=d m(\operatorname{adl})$ :

ay $O=d m(a d z)$;

set flag_out:

กOD;

$a \times 1=d m(\operatorname{adl})$ :

ay $1=\square m(\operatorname{adz})$;

reset flag_out:

¿convert samole from adl)

ar $=0 \times f+00$ :

a $f=$ Dass ar:

$a r=a \times 1-a f$;

sr=ashift ar by $O(h i)$;

ar=pass axo:

sr=sr or ashift ar by 8 (hi):

$d m(i 2, m 0)=s r 1$ :

\{convert samole from adz

ar $=0 \times f+00$ :

ar=ay $1-2 r$ :

sr=ashift ar by $O(h i)$ :

ar=pass ayo:

sr=sr or ashift ar by $B$ (hi):

$\exists m(i 6, m 4)=5 r 1$ :

dis sec_reg:

rti:

. endmod: 


\section{Appendix B: All-Band vs. In-Band Noise}

As discussed in Chapter 2, the signal-to-noise ratio of the incoming signal is

estimated by comparing the total received power and the power at the broadcast frequency. Parseval's Theorum shows how the in-band and wide-band powers are related.

Oppenheim (sec. 2.7) states Parseval's Theorem for the DTFT:

If

$$
x[n] \rightarrow X(\omega)
$$

then

$$
E=\sum_{n=-\infty}^{\infty}|x[n]|^{2}=\frac{1}{2 \pi} \int_{-\pi}^{\pi}|X[\omega]|^{2} d \omega
$$

gives the power in the signal.

The terms in the equation above can be compared to the measured quantities. The AD2101 does fixed-point calculations and uses a fractional number representation, which means all numbers must remain in the range $[-1,1]$. Thus to avoid overflow, the calculation of the wideband power must be normalized by the sequence length $M$ :

$$
E_{\text {wideband }}=\frac{1}{M} \sum_{n=-\infty}^{\infty}|x[n]|^{2}
$$

Similarly, the real and imaginary parts of the DTFT coefficient at the broadcast frequency are normalized:

$$
\begin{aligned}
& X_{\text {real }}\left(\omega_{0}\right)=\frac{1}{M} \sum_{n=\frac{M}{2}}^{\frac{M}{2}-1} x[n] \cos \left(\omega_{0} n\right) \\
& X_{\text {imag }}\left(\omega_{0}\right)=\frac{1}{M} \sum_{n=\frac{M}{2}}^{\frac{M}{2}-1} x[n] \sin \left(\omega_{0} n\right)
\end{aligned}
$$


Thus the in-band power calculated is :

$$
E_{\text {in-band }}=X_{\text {real }}{ }^{2}+X_{\text {imag }}^{2}=\frac{1}{M^{2}}\left|X\left(\omega_{0}\right)\right|^{2}
$$

It is easy to see that $E_{w i d e b a n d}$ is just the left-hand side of Parseval's Equality, normalized by $M$. The in-band power is related, although less directly, to the right-hand side. The DSP chip calculates the power at the broadcast frequency only, while Parseval's Equability deals with the power integrated over the whole spectrum. The ratio

$$
\frac{\left.1 \mathrm{X}\left(\omega_{0}\right)\right|^{2}}{2 \pi \int_{-\pi}^{\pi}|X[\omega]|^{2} \mathrm{~d} \omega}
$$

must be known to relate the measured powers.

If there is only signal present and no noise, the ratio above will be determined by the smearing effects of the window used. The rectangular windowing function has the Fourier transform (Oppenheim, section 2.7):

$$
\operatorname{Rect}[n]=\frac{\sin [\omega(M+1) / 2]}{\sin (\omega / 2)} e^{-j \omega M} / 2
$$

where $M$ is again the sequence length. Taking the limit as $\omega$ goes to zero, $W(\omega)$ goes to $\mathrm{M}+1$, so the numerator of the above ratio is

$$
\left|\mathrm{X}\left(\mathrm{ej} \omega_{0}\right)\right|^{2}=(\mathrm{M}+1)^{2}
$$

The integral of the power over the spectrum is found by rewriting the sine as a rational function in the $\mathrm{z}$ plane (the phase term disappears as this is a power calculation) and integrating around a contour. Omitting the details here, the result is obtained

$$
\frac{1}{2 \pi} \int_{-\pi}^{\pi}\left(\frac{\sin \left[\frac{\omega(M+1)}{2}\right]}{\sin \left(\frac{\omega}{2}\right)}\right)^{2} d \omega=M+1
$$

This gives the ratio from above: 


$$
\frac{\left|X\left(\omega_{0}\right)\right|^{2}}{\left.\frac{1}{2 \pi} \int_{-\pi}^{\pi} \mid X[\omega]\right]^{2} d \omega}
$$

Normalizing Parseval's Equation and substituting the above expression gives

$$
\frac{1}{M} \sum_{n=-\infty}^{\infty}|x[n]|^{2}=\frac{1}{2 \pi M} \int_{-\pi}^{\pi}|X[\omega]|^{2} d \omega=\frac{2}{M(M+1)} \mid X\left(\omega_{0}\right)^{2}
$$

or equivalently,

$$
E_{\text {wideband }}=\frac{2 M}{M+1} E_{\text {in-band }}
$$

The factor of 2 accounts for the fact that mathematically there is an energy spike at the negative frequency - $\omega 0$ equal to the spike at $\omega 0$.

In-lab tests were carried out to see how well the measured ratio of in-band to wideband power compares to the calculated ratio. Input voltages were measured on an oscilloscope and the squared rms voltage was taken as a power measure (the oscilloscope and A/D's used have identical input impedances, so there is no need to scale observed voltages by impedance in order to estimate power). The signals were then input to the DSP system, and estimates of wideband and in-band power were recorded as shown below. The A/D converters used have a $10 \mathrm{~V}$ scale, meaning the wideband power estimates should be multiplied by 100 before comparison with the oscilloscope measurements. The tests were carried out for a sequence length of $M=48$, which was normalized by 50 to prevent overflow. Substituting into the equation above leads to an expected ratio of 2.13.

As seen in the table below, the measured ratios tend to be slightly higher than predicted. Also, it can be seen that the oscilloscope measurement and the power measured by the DSP are in only rough agreement. The reasons for these errors are not clear. It should be noted that the square-and-sum power calculation is susceptible to arithmetic error from the fixed-point roundoff, which could account for some of the disagreements between 
oscilloscope and DSP measurements. Still, it can be seen that the power measurement is at least roughly correct, and should be serviceable for detection purposes.

Table B.1 - Measured Powers and Ratios

\begin{tabular}{|c|c|c|c|}
\hline $\begin{array}{c}\text { Power }\left(\mathrm{Vrms}^{2}\right) \\
\text { Measured by } \\
\text { Oscilloscope }\end{array}$ & $\begin{array}{c}\text { Wide-Band Power - } \\
\text { DSP }\end{array}$ & $\begin{array}{c}\text { In-band } \\
\text { Power - DSP }\end{array}$ & $\begin{array}{c}\text { Ratio of } \\
\text { Wide/In-band Power }\end{array}$ \\
\hline & 0.1932 & 0.0869 & 2.22 \\
18.91 & 0.0781 & 0.0357 & 2.18 \\
9.90 & 0.0466 & 0.0213 & 2.18 \\
5.78 & 0.0311 & 0.0142 & 2.19 \\
4.06 & 0.0066 & 0.0030 & 2.20 \\
0.732 & 0.0011 & 0.0005 & 2.24 \\
0.101 & \multicolumn{2}{|c|}{} \\
\hline
\end{tabular}




\section{Appendix C: Phase Interference of Two Superposed Multipath Signals}

During the in-water tests, many situations were encountered in which signals arriving at the sensors along two or more ray paths interfered with each other, leading to errors in bearing estimation. In this appendix the case of two signals arriving from different directions is examined, in order to gain insight into the resulting interference.

This analysis assumes that the source and receiver are located in the same horizontal plane, and that one of the received signals will be that which travels along a direct, unrefracted path. If the amplitude of the wave is set to unity and the in-plane bearing angle is represented by $\theta$, the wave can be written

$$
\begin{aligned}
& p_{d}=\sin \left(k_{x} x+k_{y} y+\omega t\right) \\
= & \sin (k \cos \theta x+k \sin \theta y+\omega t)
\end{aligned}
$$

The other arrival considered impinges on the hydrophones at some vertical angle $\varphi$. If this path consists of a perfect reflection off the water surface, then the amplitude will be the same as for the direct path and a phase shift of $\pi$ is introduced. This ray path will have a different path length than the direct path, which leads to a phase shift between the paths. This phase shift can be combined with that due to reflection to yield a net phase shift $\Phi_{\Delta \mathrm{r}}$, giving

$$
\begin{gathered}
p_{r}=\sin \left(k_{x} x+k_{y} y+k_{z} y+\omega t+\phi_{\Delta r}\right) \\
=\sin \left((k \sin \varphi \cos \theta) x+(k \sin \varphi \sin \theta) y+(k \cos \varphi) z+\omega t+\phi_{\Delta r}\right)
\end{gathered}
$$

It should be noted that $\phi \Delta \mathrm{r}$ depends on the path length difference, which will typically be many wavelengths long. A moving surface will therefore introduce changes in the path length that are sizable compared to a wavelength, and will cause large changes in the relative phase shift. Thus the phase shift $\phi \Delta \mathrm{r}$ can be expected to be fairly random for inwater tests. (Note this will also be true of bottom-reflected paths, if the source or receiver are moving relative to the bottom). 
The superposition of the two arrivals can now be calculated simply. The trigonometric formula in Eq. (C.3) can be used to find the resulting waveform.

$$
\sin z_{1}+\sin z_{2}=2 \sin \left(\frac{z_{1}+z_{2}}{2}\right) \cos \left(\frac{z_{1}-z_{2}}{2}\right)
$$

This yields the resultant signal received at the hydrophones, seen in Eq. (4).

$$
\begin{gathered}
p_{d}+p_{r}=2 \cos \left(\frac{k \cos \theta(1-\sin \varphi)}{2} x+\frac{k \sin \theta(1-\sin \varphi)}{2} y-\frac{k \cos \varphi}{2} z-\frac{\phi_{\Delta r}}{2}\right) . \\
\sin \left(\frac{k \cos \theta(1+\sin \varphi)}{2} x+\frac{k \sin \theta(1+\sin \varphi)}{2} y+\frac{k \cos \varphi}{2} z+\omega t+\frac{\phi_{\Delta r}}{2}\right)
\end{gathered}
$$

A few observations can now be made. The cosine term above has no time dependence, and represents an amplitude modulation of the waveform due to interference. This amplitude term depends on the geometry of the situation, but also on the relative phase shift $\phi \Delta r$ between the channels. Since this phase shift is random for most experimental situations, the signal amplitude can also be expected to be random.

The effect of interference on the bearing estimate can now be examined. As shown in Chapter 2, the measure phase difference between the pair of hydrophones oriented along the $y$-axis is

$$
\Delta \phi_{\mathrm{y}}=\mathrm{k}_{\mathrm{y}} \mathrm{D}
$$

The term multiplying the $y$-variable in Eq. (C.4) can be substituted in for $\mathrm{k}_{\mathrm{y}}$. If a halfwavelength hydrophone spacing is used, simple algebra shows that the bearing angle is given by

$$
\theta=\operatorname{Arcsin}\left(\frac{2 \Delta \phi_{y}}{\pi(1+\sin \varphi)}\right)
$$

If the assumption is made that all arrivals are in the plane of the array, $\varphi=90^{\circ}$ and the equation reduces to

$$
\theta=\operatorname{Arcsin}\left(\frac{\Delta \phi_{y}}{\pi}\right)
$$

This result allows interpretation of some of the test results in Chapter 5. In the long-range results in particular it was seen that reasonable bearing estimates could be obtained during sections of the pulse for which interference was present, but the interfering paths were incident at a small angle from the horizontal. The analysis above offers an 
explanation for these observations. All bearing angles were computed using Eq. (C.7), and therefore should be somewhat in error When the angle $\varphi$ is close to $90^{\circ}$, however, the value of $\sin \varphi$ is relatively stable and the differences between Eq.(C.6) and (C.7) are not large. 


\section{Appendix D: Wideband USBL}

\section{D.0 Overview}

All of the work presented in this thesis has assumed that the waveform transmitted by the acoustic beacon is a single-frequency pulse. During the early stages of the development process the use of other waveforms, such as FM chirps or coded noise-like sequences, was explored. In this appendix a possible processing scheme for using large bandwidth signals is presented, and its advantages and disadvantages are discussed.

\section{D.1 Pulse Detection}

Large bandwidth signals are generally detected using a correlation, or matched filter, receiver. In this detection scheme the signal received at the sensors is convolved with a time-reversed copy of the transmitted pulse. This convolution is represented as:

$$
z(t)=\int_{\tau_{1}}^{\tau_{2}} x(\tau) s(t-\tau) d \tau
$$

If the waveform is chosen carefully, the output of the correlator will be quite low at all times except when a pulse is received. The correlator output is compared to a threshold to determine when a pulse is present.

A large body of theory describing matched filter detection exists and could be exploited for a wideband USBL system. An excellent discussion of the topic is found in Van Trees (1971). With the advent of more powerful processing technologies, attention has focused recently on the advantages of coded noise-like waveforms [for example, Halsema , 1992]. The advantages of these waveforms will be discussed below. 


\section{D.2 Bearing Estimation}

In Chapter 2, it was shown that the bearing measurement could be written in terms of the time delays between arrivals at the different hydrophones. For the single-frequency processing, this time delay was rewritten in terms of the resulting phase difference. In the wideband case the time delay is estimated by estimating the phase shift between channels over a range of frequencies. An excellent overview of the problem is found in Carter [1981].

\section{D.2.1 Signal Processing}

There are several approaches used to estimating time delay for wideband signals. The most straightforward approach is to implement matched filter detection on each channel, with extremely fine resolution in time. If the processing is implemented digitally, this means the incoming signal must be sampled at an extremely high sample rate (tens of $\mathrm{MHz}$ ) in order to obtain sufficient resolution. Nautronix Ltd., an Australian firm, sells a USBL system which operates on this principle. Nautronix claims excellent accuracy, at $.25 \%$ of slant range and $.15^{\circ} \mathrm{rms}$ bearing error. However, A/D converters capable of obtaining the necessary speeds tend to have high power requirements. Thus the high time resolution correlation detector approach is not suitable for AUV operations.

Time delay for wideband signals can also be estimated using spectral estimation techniques. This approach is detailed in a number of papers [Piersol, 1981, and Chan, Hattin, and Plant, 1978]. If only two sensors are present, a received pulse will be seen on each as:

$$
\begin{gathered}
\mathrm{x}_{1}(\mathrm{t})=\mathrm{s}(\mathrm{t})+\mathrm{n}_{1}(\mathrm{t}) \\
\mathrm{x}_{2}(\mathrm{t})=\mathrm{s}(\mathrm{t}+\delta \mathrm{t})+\mathrm{n}_{2}(\mathrm{t})
\end{gathered}
$$

where $\mathrm{s}(\mathrm{t})$ is the uncontaminated signal, $\delta \mathrm{t}$ is the time delay between the sensors, and $\mathrm{n} 1$ and $\mathrm{n}_{2}$ are zero-mean Gaussian random processes which are uncorrelated with each other or the signal. The power cross-spectrum

$$
\mathrm{G}_{\mathrm{x}_{1} \mathrm{x}_{2}}(\omega)=\mathrm{X}_{1}(\omega) \mathrm{X}_{2}^{*}(\omega)
$$


and the magnitude-square coherence

$$
\mid \hat{\gamma}^{2}=\frac{\left|\mathrm{X}_{1}(\omega) \mathrm{X}_{2}^{*}(\omega)\right|^{2}}{\left|\mathrm{X}_{1}(\omega)\right|^{2}\left|\mathrm{X}_{1}(\omega)\right|^{2}}
$$

are then calculated. If no noise is present, the cross-spectrum is given by

$$
G_{x_{1} x_{2}}(\omega)=S(\omega) S^{*}(\omega) e^{j \omega \delta t}
$$

where the exponential phase shift term comes from the time delay in the second signal. The phase of the cross-spectrum can then be examined. If the sound channel was nondispersive, the time delay $\delta t$ will be constant for all frequencies. The phase term can be plotted vs. frequency, and will form a straight line. This is shown in Figure D1. The slope of the line can be calculated from the data, and the time delay found form the slope. The only assumption made about the sampling rate in this analysis is that the Nyquist rate is satisfied. Therefore, $\mathrm{A} / \mathrm{D}$ converter rates on the order of $100 \mathrm{kHz}$ are generally acceptable for USBL systems, leading to large power savings over the direct correlation detection method.

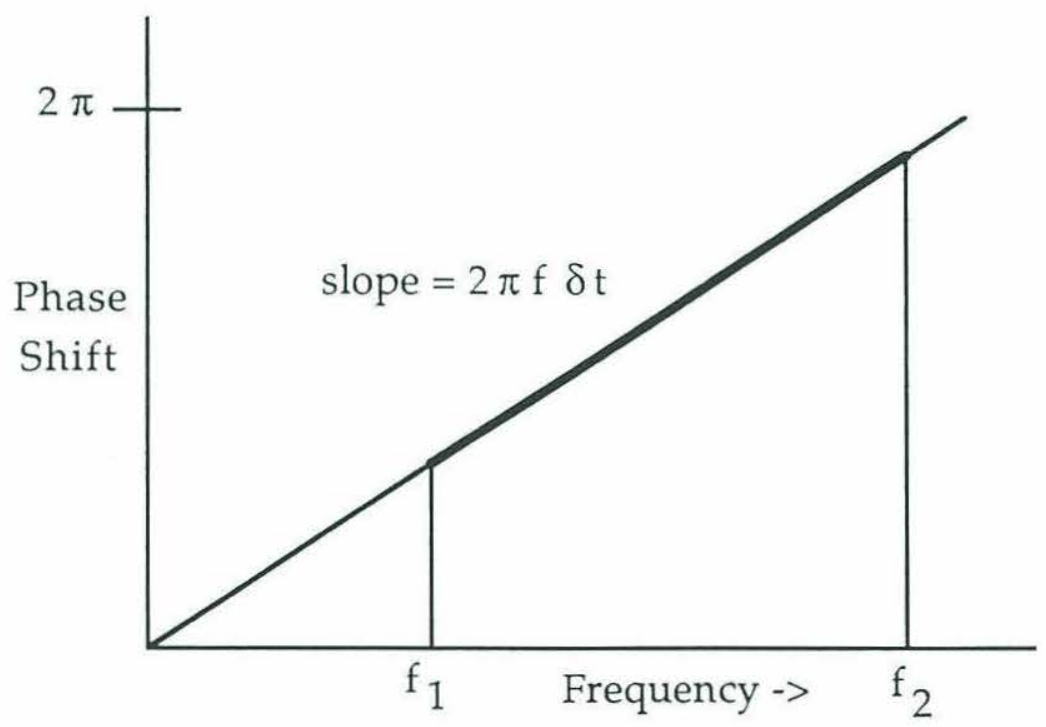

Figure D1: Time Delay Estimation Using Spectral Estimate

When noise is present, the data points will be scattered about the line shown above. The phase at each frequency is then given by 


$$
\phi=\omega \delta \mathrm{t}+\varepsilon
$$

where $\varepsilon$ is a random variable whose properties depend on the windowing and averaging done to form the estimate of the cross-spectrum. The expected error and closeness of fit can be directly related to the estimated coherence. Thus the coherence can be used as a statistic for detection [Chan, Hattin, and Plant, 1978].

This processing approach can be extended in several interesting ways. If the sound channel is dispersive, then the relation between phase shift and frequency will not be linear. If the dispersion can be predicted, then the data can be fit to the expected frequency - phase shift curve. Alternatively, the shape of the frequency - phase shift plot could be used to extract information about dispersion in the sound channel.

Ocean ambient noise is of course not uncorrelated in space and time, as assumed above. The correlation structure of the noise will then cause deviations from the expected frequency - phase shift plot. In many cases a reasonably accurate model for the spatial coherence of the noise field may be obtained. The model can be incorporated into the processing to correct for noise coherence [Piersol, 1981].

\section{D.2.2 Bearing Error Predictions}

The performance of the wideband bearing estimation method can be easily calculated. If the received signal is broken up into a large number of subsections and averaged in forming the spectral estimate, then the error $\varepsilon$ above is well approximated as a Gaussian variable [Chan, Hattin, and Plant, 1978]. The coherence and resulting bearing error can then be directly related to the SNR. The error curves below were generated using this assumption.

Figure D2 compares predicted errors for a single-frequency USBL system with errors for a wideband USBL operating with of $24.997-25 \mathrm{kHz}, 24-25 \mathrm{kHz}, 20-25 \mathrm{kHz}$, and $15-25 \mathrm{kHz}$. Spherical spreading was assumed, and attenuation of the sound be sea water was accounted for. A total signal power of $180 \mathrm{~dB}$ re $1 \mu \mathrm{Pa}$ was used. An ambient 
noise level of $55 \mathrm{~dB}$ re $1 \mu \mathrm{Pa}$ was assumed, and electronics noise was assumed to be small compared to ambient noise. The sensor separation was set to avoid aliasing at the highest frequency used.

As can be seen, the $3 \mathrm{~Hz}$ bandwidth prediction agrees fairly well with the error estimate for single-frequency processing. This provides a check on the error calculation, since the wideband and single-frequency error predictions should converge in the limit as bandwidth goes to zero.

The other curves show that bearing error increases with range. This is not particularly surprising, since increasing the signal bandwidth also increases the noise bandwidth. The total signal energy put into the water was assumed to be constant regardless of the bandwidth, so the SNR drops with increasing bandwidth. This poorer bearing performance does not necessarily mean that a single-frequency USBL system always represents a better choice than a wideband USBL. As bandwidth increases the time resolution and processing gain for detection will increase, and these gains may offset the losses in bearing accuracy.

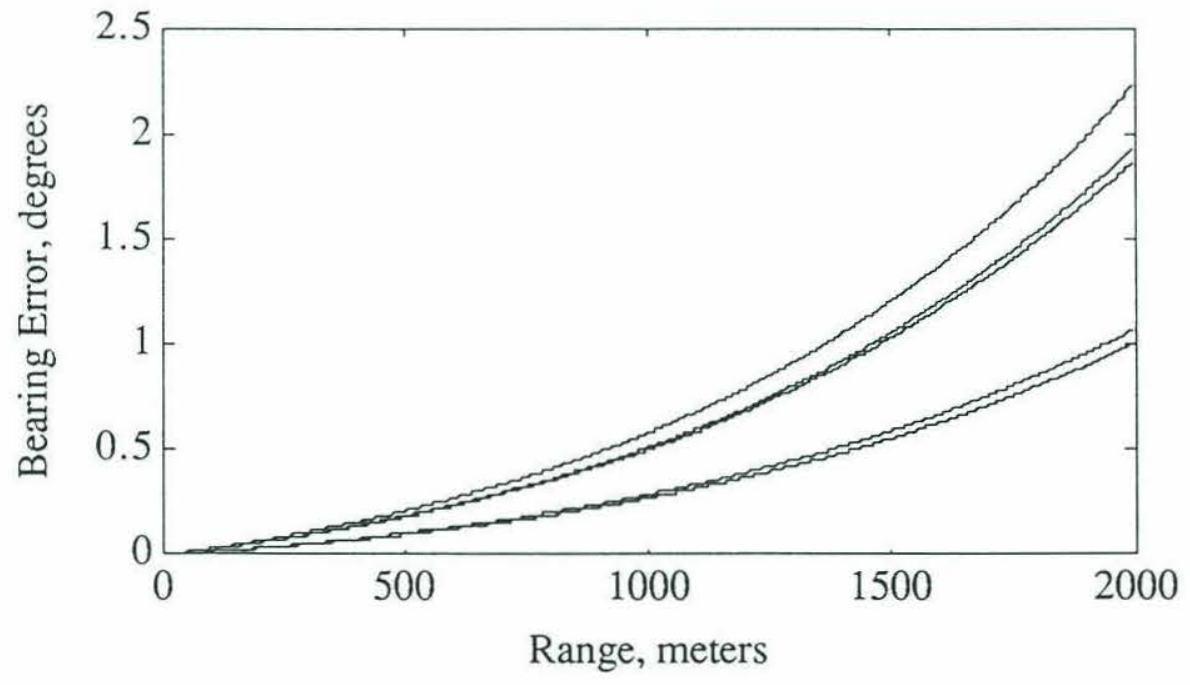

Figure D2: Predicted Bearing Error Vs Range for Different Bandwidth Signals bottommost line $=$ single frequency error estimate other estimates, listed from bottom to top, correspond to $3 \mathrm{~Hz}$, $1 \mathrm{kHz}, 5 \mathrm{kHz}$, and $10 \mathrm{kHz}$ bandwidth 


\section{D.3 Performance Improvements from Sensor Spacing}

In Chapter 2 it was shown that bearing ambiguities result for a single-frequency system if at least one pair of hydrophone is unaliased, i.e. separated by $\lambda / 2$ or less. The bearing error plots shown above also assumed an unaliased hydrophone pair was used. However, this constraint on sensor spacing does not necessarily apply for wideband USBL systems. If the hydrophone spacing is wider than $\lambda / 2$, where $\lambda$ is set by the highest frequency, the phase shift will exceed $2 \pi$. This wraparound is seen in Fig. D3 below. It is a simple matter to add code in the processing which checks for phase wraparound. This wraparound can be straightened out, and the slope of the line and time delay can be estimated.

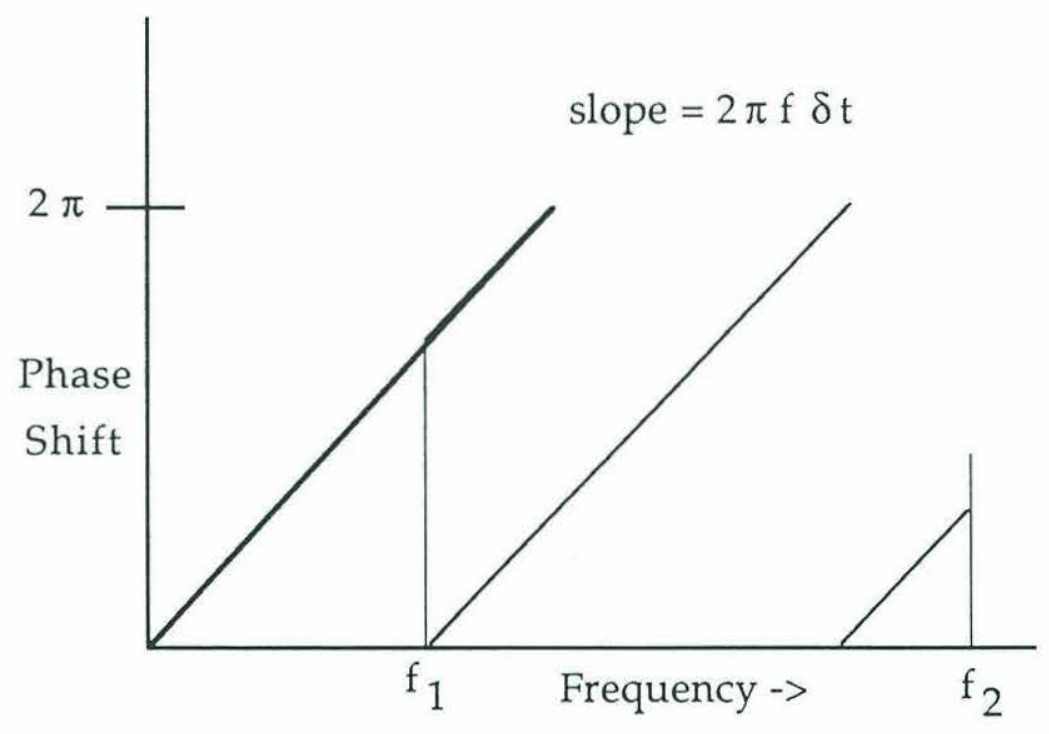

Figure D3: Effect of Spatial Aliasing on Wideband Phase Difference

This flexibility in sensor spacing can lead to substantial improvements in bearing estimation. Figure D4 below shows predicted bearing errors vs. range for hydrophone separations of $\lambda / 2, \lambda$, and $2 \lambda$, compared to errors for a single-frequency USBL system. 


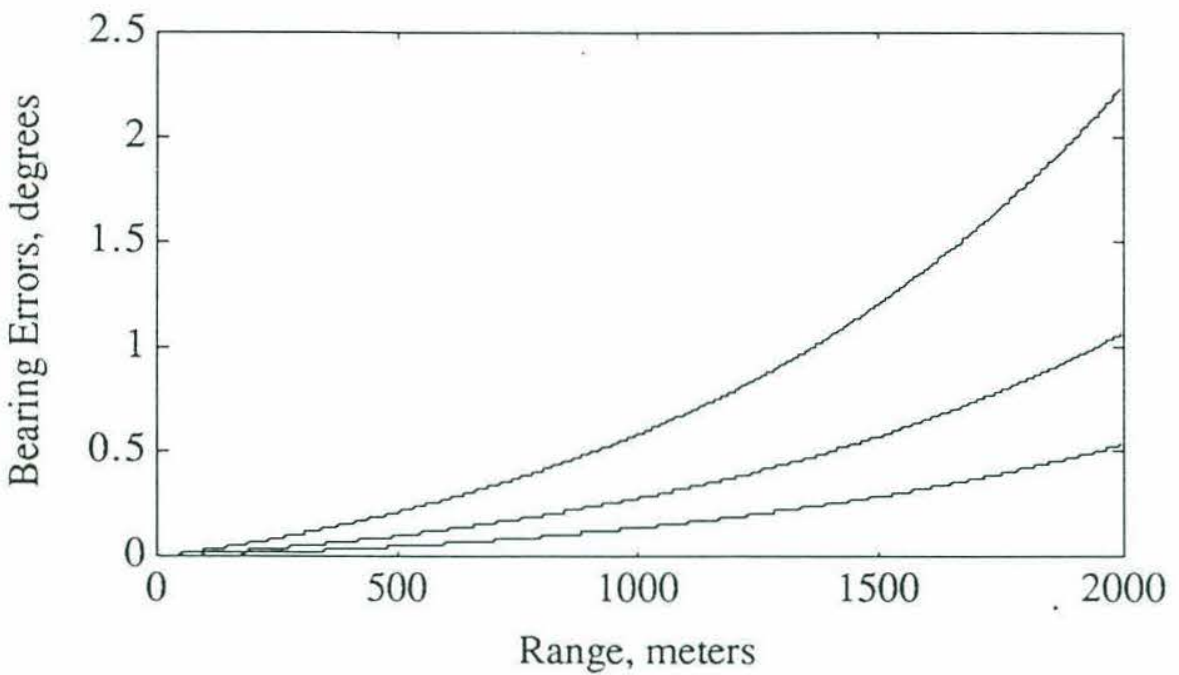

Figure D4: Bearing Error Vs Range for $15-25 \mathrm{kHz}$ Signal, Different Sensor Spacings top line $=\lambda / 2$ separation, middle $=\lambda$ separation, bottom $=2 \lambda$ separation

\section{D.4 Possible Development for USBL system}

The discussion above suggests that wideband USBL is a promising technique. A number of topics need to be more fully researched in order for this technique to be most effectively applied. Accurate models for spatial coherence of ambient noise need to be developed and incorporated in the processing. The expected effects of surface and bottom interactions on signal coherence could be calculated and used to identify the paths along which received signals have traveled. The Nautronix system has an ability to discriminate between a direct-path and surface-bounce arrival by looking at the shape of the received pulse [Russ Stewart, verbal communication, July '91]. Any information obtained about ray paths could be utilized by a matched field processing scheme.

Careful thought should be given to the choice of wideband signal used. Coded noise-like signals can be constructed which have less problems with range-Doppler ambiguity than chirp signals [Halsema, 1992]. In addition, coded noise-like pulses can be broken up into small subsections, each one of which will contain the full frequency bandwidth of the signal. In contrast, it is necessary to process the entire duration of a chirp 
signal in order get benefit of the full bandwidth. During tests in a crowded harbor there would be a high probability that reflections off the water surface, nearby ships, docks, etc. could lead to interfering overlapped multipaths. Overlapped multipath could serious problems for a chirp system, which could be avoided by using a coded signal and processing the received pulse in small segments. 


\section{References}

Bendat, J.S., and Piersol, A.G., Random Data: Analysis and Measurement Procedures, 2nd Edition, John Wiley \& Sons, New York, 1986.

Carter, G. Clifford., "Time Delay Estimation for Passive Sonar Signal Processing", p. 463-470, IEEE Transactions of Acoustics, Speech, and Signal Processing, Vol ASSP-29, No. 3, June 1981.

Chan, Y.T., Hattin, R.V., and Plant, J.B., "The least squares estimation of time delay and its use in signal detection", p. 217-222, IEEE Transactions of Acoustics, Speech, and Signal Processing, Vol ASSP-26, No. 3, June 1978.

Chan, Y.T., Riley, J.M.F., and Plant, J.B., "Modeling of time delay and its application to estimation of nonstationary delays", p. 577-581, IEEE Transactions of Acoustics, Speech, and Signal Processing, Vol ASSP-29, No. 3, June 1981.

Cron, B.F., and Sherman, C.H., "Spatial-correlation functions for various noise models", p. 1732-1736, Journal of the Acoustical Society of America, Vol. 34, No. 11, November 1962.

Del Grosso, V.A., "New equation for the speed of sound in natural waters (with comparison to other equations)", p. 1084-1091, Journal of the Acoustical Society of America, Vol 56, No. 4, October 1974. 
Farcy, A., and Leroy, C., "Two Operational Systems of Underwater Localization", Marine Technology '79.

Graf, Rudolf, Encyclopedia of Electronic Circuits, Vol. 1, Tab Books, Blue Rige Summit, PA., 1985.

Halsema, John A., "A High Resolution Wide-Band Sonar using Coded Noise-like Waveforms and a Parametric Tranmit Array", Ocean Engineer Thesis, Massachusetts Institute of Technology, February 1992.

Hamon, B.V. and Hannan, E.J., "Spectal estimation of time delay for dispersive and nondispersive systems", p. 134-142, Applied Statistics, Vol. 23, No. 2, 1974.

Haykin, Simon, Communications Systems, 2nd Edition, John Wiley and Sons, 1983.

Henderson, T.L., "Wide-band monopulse sonar: Processor performance in the remote profiling application", IEEE J. Oceanic Eng., vol OE-12, pp. 182-197, Jan. 1987.

Horowitz, Paul, and Hill, Winfield, The Art of Electronics, Cambridge University Press, Cambridge, U.K., 1980.

Huiyong, L., "An Auxiliary Navigation System for an Autonomous Underwater Vehicle", I.E.E.E./M.T.S., Proceeding Oceans '84, pp. 604-609, 1984.

Ithaco Scientific Instruments Catalog, Ithaco, Inc., Ithaca, NY, May 1988. 
Kuperman, W.A., and Ingenito, F., "Spatial correlation of surface generated noise in a stratified ocean", p.1988-1996, Journal of the Acoustical Society of America, Vol 67, No. 6, October 1980.

Light, Russell, "Seashuttle: Sonar Target Now a Robotic Underwater Vehicle", Univ. of Washington Applied Physics Lab, 1988.

Meiksin, Z.H., and Thackray, Philip C., Electronic Design With Off-The-Shelf Integrated Circuits, Prentice-Hall Inc., Englewood Cliffs, N.J., Second Edition, 1984.

Oppenheim, A., and Schafer, R., Discrete-Time Signal Processing, Prentice Hall, 1989.

Piersol, Allan G., "Time delay estimation using phase data", p. 471-477, IEEE Transactions of Acoustics, Speech, and Signal Processing, Vol ASSP-29, No. 3, June 1981.

Rich, Alan, "Shielding and Guarding", p. 124, The Best of Analog Dialogue, Analog Devices, Norwood, MA, 1991.

Rich, Alan, "Understanding Interference-Type Noise", p. 120, The Best of Analog Dialogue, Analog Devices, Norwood, MA, 1991.

Scakman, G.L., and Shelef, S.C., "The use of phase difference trace functions for bearing estimation with small circular arays", p. 501-507, IEEE Transactions of Acoustics, Speech, and Signal Processing, Vol ASSP-29, No. 3, June 1981. 
Schmidt, H., Bellingham, J. and Consi, T., "A Practical Navigation System for Autonomous Robotic Submersibles", MIT Sea Grant Memo, April 1990.

Strovik, I.M., "Hydroacoustic Positioning System", Simrad Subsea A/S, Subtech '83.

Triantafyllou, M., and Streitlien, K., "Distributed Control of Multiple AUV Forming Effective Chains", 7th Symposium on Unmanned Untethered Submersible Technology, September, 1991.

Urick, Robert J., Principles of Underwater Sound, 3rd Edition, McGraw-Hill Book Co, New York, 1983.

Van Trees, H. L., Detection, Estimation, and Modulation Theory, John Wiley and Sons, 1971.

Vestgaard, K., Hansen, K., "Super Short Baseline Hydroacoustic Navigation System", Simrad Subsea A/S, Tenth Offshore Technology Conference, 1978.

Vestgaard, K., Jacobsen, H.P., Klepaker, R.A., Christensson, C.G., "Super Short Baseline Hydroacoustic Navigation System", Simrad Subsea A/S, ROV '84.

Watson, M., Berkowitz, J., and Wapner, M., "Ultra-Short Baseline Acoustic Tracking System", Sonatech, Inc., Oceans '83.

Willsky, Alan. S., and Shapiro, Jeffrey. H., Stochastic Processes, Detection, and Estimation: Supplementary Notes, for MIT course, 1988. 